\title{
Robust Multi-product Newsvendor Model with Uncertain Demand and Substitution
}

\author{
Jie Zhanga,*, Weijun Xie ${ }^{\mathrm{a}}$, Subhash C. Sarin ${ }^{\mathrm{a}}$ \\ ${ }^{a}$ Department of Industrial and Systems Engineering, Virginia Tech, Blacksburg, VA 24061
}

\begin{abstract}
This work studies a Robust Multi-product Newsvendor Model with Substitution (R-MNMS), where the demand and the substitution rates are stochastic and are subject to cardinality-constrained uncertainty sets. The goal of this work is to determine the optimal order quantities of multiple products to maximize the worst-case total profit. To achieve this, we first show that for given order quantities, computing the worst-case total profit, in general, is NP-hard. Therefore, we derive the closed-form optimal solutions for the following three special cases: (1) if there are only two products, (2) if there is no substitution among different products, and (3) if the budget of demand uncertainty is equal to the number of products. For a general R-MNMS, we formulate it as a mixed-integer linear program with an exponential number of constraints and develop a branch and cut algorithm to solve it. For large-scale problem instances, we further propose a conservative approximation of R-MNMS and prove that under some certain conditions, this conservative approximation yields an exact optimal solution to R-MNMS. The numerical study demonstrates the effectiveness of the proposed approaches and the robustness of our model.
\end{abstract}

Keywords: Stochastic programming, robust, cardinality-constrained uncertainty set, mixed-integer program, branch and cut algorithm

\section{Introduction}

This work studies Multi-product Newsvendor Model with Substitution (MNMS) under demand and substitution rate uncertainty, in which a retailer determines the optimal order quantity for each product to maximize its total profit. Due to similarity among different products and their occasional unavailability, substitution among different products is quite common and has been observed in many studies (Bassok et al., 1999, Rajaram and Tang, 2001; Chopra and Meindl, 2007: Shumsky and Zhang, 2009, Stavrulaki, 2011, Choi, 2012, Yu et al., 2015). For instance, when shopping at Amazon.com, a customer might turn to a blue hat if a green hat, his or her first-choice,

\footnotetext{
${ }^{*}$ Corresponding author

Email addresses: jiezhang@vt.edu (Jie Zhang), wxie@vt.edu (Weijun Xie), sarin@vt.edu (Subhash C. Sarin)
} 
were currently unavailable. Substitution somehow increases the profit of the retailer (Rajaram and Tang, 2001), but on the other hand, significantly complicates the problem and makes the problem very challenging to handle. Besides, due to the stochasticity of customers' demand and substitution rates, it might be hard to forecast the demand and substitution rates accurately. Therefore, many works (Erlebacher, 2000; Schweitzer and Cachon, 2000; Rajaram and Tang, 2001; Rao et al., 2004, Zhang et al. 2018) proposed stochastic programming models to tackle the demand uncertainty by assuming that the probability distribution of the demand is known. However, in many cases, having a good estimation of probability distribution might be very challenging. In particular, nowadays, technology companies, and original equipment manufacturers frequently release their new products. For example, every year, Apple Inc. releases its new-generation iPhones and MacBooks. Without enough historical sales data, it is almost impossible to have an accurate prediction of these new products' demand and substitution rates and inaccurate estimations can cause misleading decisions (Xie and Ahmed, 2018a). Therefore, to foster a more reliable decision, instead, we study the "Robust" Multi-product Newsvendor Model with Substitution (R-MNMS) subject to cardinality-constrained uncertainty set.

R-MNMS has the following technical features. First of all, due to the substitution effect, it has been shown in Zhang et al. (2018), even when the demand is deterministic, MNMS can be NP-hard. Second, most of the existing works assumed that the customers' demand follows a given probability distribution, which, however, might result in a loss of sales due to inaccurate demand forecasting. Third, although many existing works illustrated interesting properties of MNMS, the closed-form optimal solutions are rarely known; therefore, very limited managerial insights have been discovered so far. In this paper, we will show that under some conditions, all of these features can be appropriately addressed.

\subsection{Relevant Literature}

This subsection reviews the relevant literature on four topics: inventory-dependent demand and product substitution, stochastic MNMS, existing work on R-MNMS, and R-MNMS under cardinality uncertainty set.

Inventory-dependent Demand and Product Substitution. Optimal inventory policy involving inventory-dependent demand has been studied extensively where the inventory level of a product can stimulate the product demand (Eliashberg and Steinberg (1993), Gerchak and Wang (1994), Balakrishnan et al. (2004), Balakrishnan et al. (2008), Baker and Urban (1988), and Loedy et al. (2018)). The substitution effects among products, that take place when a product is out-ofstock, will, in turn, influence the product demand (Maity and Maiti (2005)). Product substitution is a classical research area in inventory management. Chopra and Meindl (2007) defined product substitution as "the use of one product to satisfy demand for a different product within a specific product category. Shin et al. (2015) provides three criteria to classify product substitution: i) substitution mechanism- assortment-based substitution, inventory-based substitution, and price-based 
substitution; ii) substitution decision-maker- supplier-driven substitution and customer-driven substitution; iii) Direction of substitutability- one-way substitution and two-way substitution. This work focuses on a multi-product inventory-dependent demand model with product substitution, which is similar to Huang et al. (2011), Netessine and Rudi (2003), Zhang et al. (2018), and Huang et al. (2011). Particularly, our model considers that product substitution belongs to inventorybased substitution, customer-driven substitution, and two-way substitution. More specifically, in the product substitution, customers will choose the substitutable product of the same category as the first-choice product when the substitutable product has a surplus (Stavrulaki, 2011; Rajaram and Tang, 2001).

Stochastic MNMS. For the study of MNMS, the first stream used stochastic programming approaches to handle the uncertainty in MNMS, i.e., they assumed that the probability distribution of the demand is known, for example, Huang et al. (2011), Netessine and Rudi (2003), and Zhang et al. (2018). Huang et al. (2011) analyzed the decentralized MNMS, where each retailer owns one product and competes with the other retailers assuming the conditions under which the Nash equilibrium exists, and used an iterative algorithm to solve the model. However, its centralized counterpart, where a retailer owns all the products, becomes highly non-convex, which will be studied in this paper. Netessine and Rudi (2003) demonstrated that the profit function could be quasi-concave or bi-modal when the demand is deterministic. Recently, Zhang et al. (2018) formulated stochastic MNMS as a mixed-integer linear program and developed polynomial-time approximation algorithms with performance guarantees to solve it. Distinct from these works, this study addresses centralized R-MNMS under a cardinality-constrained uncertainty set.

Existing Works on R-MNMS. However, in practice, It is not trivial to determine the distribution of the random demand; in particular, when the random demand is not stationary, i.e., the probability distribution of the random demand subjects to changing from time to time. This inaccurate probability distribution could result in poor decisions. Under these circumstances, alternatively, the second stream chose the robust approach (i.e., R-MNMS) to formulate the model with partial information of the demand, which can be easily characterized or will stay the same at a relatively long period (i.e., mean, variance, or support). For decentralized R-MNMS, Jiang et al. (2011) used the absolute regret criterion to obtain the unique Nash equilibrium. Only the support of the demand is known in their work, and they also showed that the robust model tended to be more tractable than the stochastic counterpart. Recent work in $\mathrm{Li}$ and Fu (2017) studied a robust two-product newsvendor model with substitution when the first two moments of demand are known. However, the authors were only able to provide an optimal solution for two extreme cases: (1) no substitution, or (2) perfect substitution between products. Unlike these works, our assumptions are much less restrictive and we also study centralized R-MNMS with more than two products.

R-MNMS under Cardinality-Constrained Uncertainty Set. In this paper, we study RMNMS using the cardinality-constrained uncertainty set to characterize the random demand and 
substitution rates. The cardinality-constrained uncertainty set was first introduced by Bertsimas and Sim (2004) into robust optimization to reduce over-conservatism while, at the same time, achieving robustness. This framework has been successfully applied to healthcare (Lanzarone and Matta, 2012; Carello and Lanzarone, 2014, Addis et al., 2015), manufacturing (Lugaresi, 2016. Lugaresi et al., 2017), inventory management (Bertsimas and Thiele, 2006; Solyalı et al., 2012), portfolio optimization (Moon and Yao, 2011), scheduling (HazlR and Dolgui, 2013; Lu et al., 2014 Moreira et al. 2015), etc. To our knowledge, we are the first to study R-MNMS under the cardinality-constrained uncertainty set with random demand and substitution rates. We analyze the complexity of R-MNMS and develop the exact and approximation algorithms to solve the model. Moreover, we derive closed-form solutions for three different special cases. Finally, the numerical study demonstrates the robustness of the solutions of R-MNMS.

\subsection{Summary of Main Contributions and Managerial Insights}

The objective of this study is to provide a retailer the ability to determine optimal order quantities in a single-period multi-product newsvendor model with substitution, which optimizes the worst-case total profit under the cardinality-constrained uncertainty set. Our contributions are summarized as below.

We develop an equivalent reformulation of R-MNMS and prove that computing the worstcase total profit, in general, is NP-hard for given order quantities via a reduction to the clique problem. This result leads us to develop approximation algorithms for the general R-MNMS and exact closed-form solutions for special cases of R-MNMS. The complexity analysis of R-MNMS can also enlighten researchers to the properties of R-MNMS with other robust settings and develop the corresponding solution approaches.

Although solving R-MNMS, in general, is NP-hard, we derive closed-form solutions for the following three special cases of R-MNMS: (I) if there are only two products; (II) if there is no substitution among different products; or (III) if the budget of demand uncertainty is equal to the number of products. In case (I), we suggest that the decision makers should only order the product with a higher marginal profit and substitute the other product. In case (II), the decisionmakers should be conservative to avoid the loss from inaccurate demand forecasting. In case (III), the decision-makers should order up to the effective demand of a product if its marginal profit is relatively high and not order it otherwise.

For the general R-MNMS, we reformulate it as a mixed-integer linear program (MILP) with an exponential number of constraints and develop branch and cut algorithm to solve it. This approach may inspire researchers to solve R-MNMS with other uncertainty sets. However, to generate a valid inequality to separate an infeasible solution, one has to solve an MILP, which can be time-consuming. In pursuit of an alternative, more effective, solution method, we provide a conservative approximation of R-MNMS and prove that under the special cases (II) and (III), the proposed conservative approximation is equivalent to R-MNMS. 
Our numerical study tests the efficiency of branch and cut and conservative approximation algorithms with instances of varying sizes. Both algorithms work well when the size of the model is not large. The conservative approximation algorithm dominates large-scale instances with better solution quality. Our sensitivity result shows that the profit becomes smaller if the variance of the demand grows. We seek the best budget of uncertainty by a cross-validation method, and we also find that the solution from the robust model can be more reliable than the risk-neutral one studied in Zhang et al. (2018) when the demand data are limited.

The remainder of the paper is organized as follows. Section 2 introduces the problem setting and the model. Section 3 presents the properties of the model and proves the complexity of computing the worst-case total profit. In Section 4, we derive the optimal order quantities for three special cases of the model. Section 5 reformulates the R-MNMS as an MILP, and develops a branch and cut algorithm and a conservative approximation to solve it. Section 6 presents the results of our numerical investigation on the proposed algorithms. Section 7 concludes the paper.

Notation: The following notation is used throughout the paper. We use bold-case (e.g., $\boldsymbol{x}, \boldsymbol{A}$ ) to denote vectors and matrices and use corresponding regular-case letters to denote their components. Given a vector or matrix $\boldsymbol{x}$, its zero norm $\|\boldsymbol{x}\|_{0}$ denotes the number of its nonzero elements. We let $\boldsymbol{e}$ be the vector or matrix of all ones, and let $\boldsymbol{e}_{i}$ be the $i$ th standard basis vector. Given an integer $n$, we let $[n]:=\{1,2, \ldots, n\}$, and use $\mathbb{R}_{+}^{n}:=\left\{\boldsymbol{x} \in \mathbb{R}^{n}: x_{i} \geq 0, \forall i \in[n]\right\}$. Given a real number $t$, we let $(t)_{+}:=\max \{t, 0\}$. Given a finite set $I$, we let $|I|$ denote its cardinality. We let $\tilde{\boldsymbol{\xi}}$ denote a random vector and denote its realizations by $\boldsymbol{\xi}$. Additional notation will be introduced as needed.

\section{Model Formulation}

In this section, we present the model formulation for R-MNMS.

To begin with, suppose that there is a retailer selling $n$ similar products in the market indexed by $[n]:=\{1, \cdots, n\}$ at a given time period. For each product $i \in[n]$, its cost is $c_{i}$, price is $p_{i}$, and salvage value is $s_{i}$, where by convention, we assume that $p_{i} \geq c_{i} \geq s_{i}$. Each product also has a random demand $\tilde{D}_{i}$ for each $i \in[n]$. Ideally, the retailer would like to determine the optimal order quantity for each product $i \in[n]$, denoted as $Q_{i}$. Due to the substitution effect, the effective demand of each product will be affected by its realized demand, its order quantity as well as other products' conditions (i.e., whether out-of-stock or not). To formulate this effect, we suppose that the demand of product $j \in[n]$ can be proportionally substituted by another product $i \in[n]$ and $i \neq j$, once the part of the demand of product $j$ cannot be satisfied by its order quantity $Q_{j}$. In particular, we let $\tilde{\alpha}_{j i}$ be the substitution rate, which is the proportion of the unmet demand of product $j$ substituted by product $i$. Note that $\tilde{\alpha}_{j i}$ might not be equal to $\tilde{\alpha}_{i j}$. In this paper, we assume that all the products have the same unit of measurement, and therefore, for each pair of products $i, j \in[n]$, substitution rate satisfies $\tilde{\alpha}_{j i} \in[0,1]$. Also, by default, we let $\tilde{\alpha}_{i i}=0$ for each 
product $i \in[n]$. We let $\tilde{D}_{i}^{s}(\boldsymbol{Q})$ denote the effective demand function of product $i \in[n]$ as below:

$$
\tilde{D}_{i}^{s}(\boldsymbol{Q})=\tilde{D}_{i}+\sum_{j \in[n]} \tilde{\alpha}_{j i}\left(\tilde{D}_{j}-Q_{j}\right)_{+}, \forall i \in[n]
$$

where the second term in the sum is due to its substitution to the unavailable products.

As shown in Zhang et al. (2018), the retailer's total profit for given order quantities $\boldsymbol{Q}$, substitution rates $\tilde{\boldsymbol{\alpha}}$, and demand $\tilde{\boldsymbol{D}}$ can be formulated as:

$$
\widehat{\Pi}(\boldsymbol{Q}, \tilde{\boldsymbol{D}}, \tilde{\boldsymbol{\alpha}}):=\sum_{i \in[n]}\left(p_{i} \min \left(Q_{i}, \tilde{D}_{i}^{s}(\boldsymbol{Q})\right)-c_{i} Q_{i}+s_{i}\left(Q_{i}-\tilde{D}_{i}^{s}(\boldsymbol{Q})\right)_{+}\right) .
$$

\subsection{Constructing Uncertainty Sets of Demand and Substitution Rates}

Oftentimes, the substitution rates $(\tilde{\boldsymbol{\alpha}})$ and the demand $(\tilde{\boldsymbol{D}})$ of products in (1) are stochastic and their probability distributions are difficult to characterize. To address the uncertainties of substitution rates and the demand, we will use robust optimization. In particular, we will study R-MNMS under cardinality-constrained uncertainty sets.

First of all, in the demand uncertainty set, suppose that the demand of the $n$ products (i.e., $\tilde{\boldsymbol{D}}$ ) is within a box, e.g., $\tilde{\boldsymbol{D}} \in[\boldsymbol{D}-\boldsymbol{l}, \boldsymbol{D}+\boldsymbol{u}]$, where $\boldsymbol{D}$ denotes the nominal demand, $\boldsymbol{l}, \boldsymbol{u}$ denote the lower and upper deviations of the demand respectively satisfying $\boldsymbol{l} \in[\mathbf{0}, \boldsymbol{D}]$ and $\boldsymbol{u} \geq \mathbf{0}$. We also assume that at most $k \in[n] \cup\{0\}$ products are allowed to deviate from their nominal demand $\boldsymbol{D}$. We will discuss the impact of the budget of uncertainty $k$ on optimal order quantities. Therefore, the uncertainty set of the demand can be written as

$$
\mathcal{U}_{0}=\left\{\tilde{\boldsymbol{D}}: \tilde{D}_{i}=D_{i}+\Delta_{i},-l_{i} \leq \Delta_{i} \leq u_{i}, \forall i \in[n],\|\boldsymbol{\Delta}\|_{0} \leq k\right\}
$$

Similarly, let us denote the uncertainty set of substitution rate as below

$$
\mathcal{U}_{\alpha}=\left\{\tilde{\boldsymbol{\alpha}}: \tilde{\alpha}_{j i}=\alpha_{j i}+\Delta_{j i}^{\alpha},-l_{j i}^{\alpha} \leq \Delta_{j i}^{\alpha} \leq u_{j i}^{\alpha}, \forall i, j \in[n],\left\|\Delta^{\alpha}\right\|_{0} \leq k^{\alpha}\right\}
$$

where $\|\cdot\|_{0}$ denotes the zero-norm, and $k^{\alpha}$ is the budget of uncertainty. We suppose that the substitution rates are contained within a box, e.g., $\tilde{\boldsymbol{\alpha}} \in\left[\boldsymbol{\alpha}-\boldsymbol{l}^{\boldsymbol{\alpha}}, \boldsymbol{\alpha}+\boldsymbol{u}^{\boldsymbol{\alpha}}\right]$, where $\boldsymbol{\alpha}$ denotes the nominal substitution rates, $\boldsymbol{l}^{\alpha}, \boldsymbol{u}^{\boldsymbol{\alpha}}$ denote the lower and upper deviations of the substitution rates respectively satisfying $\boldsymbol{l}^{\boldsymbol{\alpha}} \in[\mathbf{0}, \boldsymbol{\alpha}], \boldsymbol{u}^{\alpha} \in[0, \boldsymbol{e}-\boldsymbol{\alpha}]$. For notational convenience, we let $\alpha_{i i}^{\alpha}=l_{i i}^{\alpha}=$ $u_{i i}^{\alpha}=0$ for each $i \in[n]$.

With the notation introduced above, R-MNMS can be formulated as:

$$
v^{*}=\max _{\boldsymbol{Q} \in \mathbb{R}_{+}^{n}} \min _{\tilde{\boldsymbol{D}} \in \mathcal{U}_{0}, \tilde{\boldsymbol{\alpha}} \in \mathcal{U}_{\alpha}}\left\{\widehat{\Pi}(\boldsymbol{Q}, \tilde{\boldsymbol{D}}, \tilde{\boldsymbol{\alpha}}):=\sum_{i \in[n]}\left(p_{i} \min \left(Q_{i}, \tilde{D}_{i}^{s}(\boldsymbol{Q})\right)-c_{i} Q_{i}+s_{i}\left(Q_{i}-\tilde{D}_{i}^{s}(\boldsymbol{Q})\right)_{+}\right)\right\} .
$$


In Model (5), the objective is to find optimal order quantities to maximize the worst-case total profit over the uncertainty sets $\mathcal{U}_{0}, \mathcal{U}_{\alpha}$. For each product $i \in[n]$, we let $\bar{P}_{i}=p_{i}-c_{i} \geq 0$ and $\bar{S}_{i}=p_{i}-s_{i} \geq 0$. Note that $\bar{P}_{i}$ can be interpreted as the marginal profit or underage cost of product $i \in[n]$, while $\bar{S}_{i}$ is the sum of the underage cost $\left(p_{i}-c_{i}\right)$ and overage cost $\left(c_{i}-s_{i}\right)$ of product $i \in[n]$, where their ratio $\frac{\bar{P}_{i}}{\bar{S}_{i}}$ is known as the critical ratio of newsvendor model (c.f., Nahmias and Olsen $(2015))$. Since $\min \left(Q_{i}, \tilde{D}_{i}^{s}(\boldsymbol{Q})\right)=Q_{i}-\left(Q_{i}-\tilde{D}_{i}^{s}(\boldsymbol{Q})\right)_{+}$for each $i \in[n]$, the above Model (5) is equivalent to

$$
v^{*}=\max _{\boldsymbol{Q} \in \mathbb{R}_{+}^{n}} \min _{\tilde{\boldsymbol{D}} \in \mathcal{U}_{0}, \tilde{\boldsymbol{\alpha}} \in \mathcal{U}_{\alpha}}\left\{\widehat{\Pi}(\boldsymbol{Q}, \tilde{\boldsymbol{D}}, \tilde{\boldsymbol{\alpha}}):=\sum_{i \in[n]} \bar{P}_{i} Q_{i}-\sum_{i \in[n]} \bar{S}_{i}\left(Q_{i}-\tilde{D}_{i}^{s}(\boldsymbol{Q})\right)_{+}\right\} .
$$

We treat the uncertainties of demand and substitution rates separately because (i) in practice, the demand estimation and substitution rates estimation follows different procedures (c.f. Kök and Fisher (2007)) and (ii) the separable uncertainty sets allow us to reformulate the model as a mixed-integer linear program and to obtain closed-form solutions. For notational convenience, throughout this paper, we will let $\boldsymbol{Q}^{*}$ denote an optimal solution to R-MNMS (6).

\subsection{Discussion about How to Estimate the Uncertainty Sets}

The budgets of uncertainty (i.e., $k, k^{\alpha}$ ) in Model 6 plays an important role, and a good choice of these values can achieve both least-conservatism and robustness. The following steps show how to find the optimal budgets of uncertainty $k^{*}, k^{\alpha *}$ using possibly limited historical data:

Step 0: We split the historical data into two groups $\Upsilon_{i}, i \in[2]$, and select a candidate set $\mathcal{K} \subseteq\{0\} \cup[n] \times\{0\} \cup\left[n^{2}-n\right]$ to choose the best $\left(k^{*}, k^{\alpha *}\right)$.

Step 1.1: Determine nominal demand $\widehat{\mu}$, the lower deviation $l$, and the upper deviation $\boldsymbol{u}$. To do so, we compute the sample mean $\widehat{\mu}_{i}$ and standard deviation $\widehat{\sigma}_{i}$ of the first group of historical demand data $\Upsilon_{1}$ for each product $i \in[n]$. Then we set the nominal demand $D_{i}=\widehat{\mu}_{i}$, and $u_{i}=l_{i}=1.96 \widehat{\sigma}_{i}$ for each product $i \in[n]$.

Step 1.2: Determine nominal substitution rate $\widehat{\mu}^{\alpha}$, the lower deviation $\boldsymbol{l}^{\alpha}$, and the upper deviation $\boldsymbol{u}^{\alpha}$. Similarly, we compute the sample mean $\widehat{\mu}_{j i}^{\alpha}$ and standard deviation $\widehat{\sigma}_{j i}^{\alpha}$ of the first group of historical substitution rate data $\Upsilon_{1}$ for each pair of products $i, j \in[n]$. Then we set the nominal substitution rate $\alpha_{j i}=\widehat{\mu}_{j i}^{\alpha}$, and $u_{j i}^{\alpha}=l_{j i}^{\alpha}=1.96 \widehat{\sigma}_{j i}^{\alpha}$ for each pair of products $i, j \in[n]$.

Step 2: Calculate the optimal order quantities $\boldsymbol{Q}^{*}\left(k, k^{\alpha}\right)$ and objective value $v^{*}\left(k, k^{\alpha}\right)$ for each $\left(k, k^{\alpha}\right) \in \mathcal{K}$ by solving Model (6).

Step 3: Compute the objective value $\widehat{\Pi}\left(\boldsymbol{Q}^{*}\left(k, k^{\alpha}\right), \boldsymbol{D}, \boldsymbol{\alpha}\right)$ of Model 22 for each $\left(k, k^{\alpha}\right) \in \mathcal{K}$ and each pair of demand and substitution rates $(D, \alpha)$ in the second group of historical data $\Upsilon_{2}$.

Step 4: Determine the optimal $k^{*}, k^{\alpha *}$. For each $\left(k, k^{\alpha}\right) \in \mathcal{K}$, we compute the $q$ th percentile of $\left\{\widehat{\Pi}\left(\boldsymbol{Q}^{*}\left(k, k^{\alpha}\right), \boldsymbol{D}, \boldsymbol{\alpha}\right)\right\}_{(\boldsymbol{D}, \boldsymbol{\alpha}) \in \Upsilon_{2}}$, and denote it as $\widehat{\Pi}^{q \%}\left(k, k^{\alpha}\right)$. Given two nonnegative weights 
$w_{1}, w_{2} \in \mathbb{R}_{+}$, we choose the optimal budgets of uncertainty $k^{*}, k^{\alpha *}$ which achieve the smallest weighted value $w_{1} k+w_{2} k^{\alpha}$ such that $v^{*}\left(k, k^{\alpha}\right) \leq \widehat{\Pi}^{q \%}\left(k, k^{\alpha}\right)$.

\section{Equivalent Reformulation and Model Properties}

In this section, we study R-MNMS under cardinality-constrained uncertainty set and derive its equivalent reformulation. We also provide upper bounds of optimal order quantities and show that computing the worst-case total profit for given order quantities, in general, is NP-hard.

Throughout the rest of the paper, we will make the following assumption.

Assumption 1. Suppose that $k^{\alpha}=n^{2}-n$ in the substitution uncertainty set $\mathcal{U}_{\alpha}$.

Assumption 1 implies that the substitution uncertainty set $\mathcal{U}_{\alpha}$ is purely a box. We make this assumption for the following reasons: (i) first of all, it is often more difficult to estimate substitution rates $\tilde{\boldsymbol{\alpha}}$ than the demand. When the available data are limited, there might not be enough data to estimate the substitute rates. Kök and Fisher (2007) estimated the substitution rates using inventory-transactions data through the maximum likelihood function. Vaagen et al. (2011) showed that the survey data and similarity/dissimilarity analysis between products can be used to obtain substitution rates. Müller et al. (2020) adapted the methodology of Anupindi et al. (1998) and estimated the substitution rates by sales data, master data, and transaction data. The methods in the aforementioned literature are useful to estimate the substitution rates, which require more efforts than simply acquiring the demand data; (ii) second, under this assumption, we can derive some interesting analytical results; and (iii) third, our exact branch and cut algorithm in Section 5 can be applied to the general $k^{\alpha}$, and it follows directly from the derivation in Section 5.

\subsection{Equivalent Reformulation}

In this subsection, we provide an alternative formulation for Model (6).

First, we make the following observation.

Lemma 1. For any $\boldsymbol{Q}, \tilde{\boldsymbol{D}} \in \mathbb{R}_{+}^{n}$, the profit function $\widehat{\Pi}(\boldsymbol{Q}, \tilde{\boldsymbol{D}}, \cdot)$ is monotone nondecreasing in $\tilde{\boldsymbol{\alpha}}$; and for any $\boldsymbol{Q}, \tilde{\boldsymbol{\alpha}} \in \mathbb{R}_{+}^{n}$, the profit function $\widehat{\Pi}(\boldsymbol{Q}, \cdot, \tilde{\boldsymbol{\alpha}})$ is monotone nondecreasing in $\tilde{\boldsymbol{D}}$.

Proof. According to Model (6), the profit function $\widehat{\Pi}(\boldsymbol{Q}, \tilde{\boldsymbol{D}}, \cdot)$ is nondecreasing in $\tilde{D}_{i}^{s}(\boldsymbol{Q})$ and from (1), the effective demand $\tilde{D}_{i}^{s}(\boldsymbol{Q})$ is also nondecreasing in $\tilde{\alpha}_{j i}$ for each product $i, j \in[n]$. Therefore, the profit function $\widehat{\Pi}(\boldsymbol{Q}, \tilde{\boldsymbol{D}}, \cdot)$ is nondecreasing in $\tilde{\boldsymbol{\alpha}}$. Similarly, from $(1), \tilde{D}_{i}^{s}(\boldsymbol{Q})$ is also nondecreasing in $\tilde{D}_{i}$ for each product $i \in[n]$. Therefore, the profit function $\widehat{\Pi}(\boldsymbol{Q}, \cdot, \tilde{\boldsymbol{\alpha}})$ is nondecreasing in the demand $\tilde{\boldsymbol{D}}$. 
According to Lemma 1 and Assumption 1 . $\min _{\tilde{\alpha} \in \mathcal{U}_{\alpha}} \widehat{\Pi}(\boldsymbol{Q}, \cdot, \tilde{\boldsymbol{\alpha}})$ is achieved by $\tilde{\alpha}_{j i}=\alpha_{j i}-l_{j i}^{\alpha}:=$ $\underline{\alpha}_{j i}$ for all products $i \neq j$ and $i, j \in[n]$. In this case, Model (6) becomes

$$
v^{*}=\max _{\boldsymbol{Q} \in \mathbb{R}_{+}^{n}} \min _{\tilde{\boldsymbol{D}} \in \mathcal{U}_{0}}\left\{\Pi(\boldsymbol{Q}, \tilde{\boldsymbol{D}}):=\sum_{i \in[n]} \bar{P}_{i} Q_{i}-\sum_{i \in[n]} \bar{S}_{i}\left(Q_{i}-\tilde{D}_{i}^{s}(\boldsymbol{Q})\right)_{+}\right\},
$$

where we let $\Pi(\boldsymbol{Q}, \tilde{\boldsymbol{D}})=\widehat{\Pi}(\boldsymbol{Q}, \tilde{\boldsymbol{D}}, \underline{\boldsymbol{\alpha}})$.

Remark 1. If Assumption 1 does not hold (i.e., $k^{\alpha} \leq n^{2}-n$ ) and $k=n$, then according to Lemma 1, $\min _{\tilde{\boldsymbol{D}} \in \mathcal{U}_{0}} \widehat{\Pi}(\boldsymbol{Q}, \tilde{\boldsymbol{D}}, \cdot)$ is achieved by $\tilde{D}_{i}=D_{i}-l_{i}:=\underline{D}_{i}$. and $z_{i}=1, \forall i \in[n]$. The result in Lemma (7) now reads as

$$
v^{*}=\max _{\boldsymbol{Q} \in \mathbb{R}_{+}^{n}} \min _{\tilde{\boldsymbol{\alpha}} \in \mathcal{U}_{\alpha}}\left\{\Pi(\boldsymbol{Q}, \tilde{\boldsymbol{\alpha}}):=\sum_{i \in[n]} \bar{P}_{i} Q_{i}-\sum_{i \in[n]} \bar{S}_{i}\left(Q_{i}-\tilde{D}_{i}^{s}(\boldsymbol{Q})\right)_{+}\right\},
$$

where we let $\Pi(\boldsymbol{Q}, \tilde{\boldsymbol{\alpha}})=\widehat{\Pi}(\boldsymbol{Q}, \underline{\boldsymbol{D}}, \tilde{\boldsymbol{\alpha}})$.

Now we are ready to show our equivalent reformulation. The main idea of the derivation is to show that in the worst-case, the uncertainty set $\mathcal{U}_{0}$ can be restricted to the following mixed-integer set:

$$
\mathcal{U}=\left\{\tilde{\boldsymbol{D}}: \sum_{i \in[n]} z_{i} \leq k, \tilde{D}_{i}=D_{i}-l_{i} z_{i}, z_{i} \in\{0,1\}, \forall i \in[n]\right\} .
$$

Clearly, set $\mathcal{U} \subseteq \mathcal{U}_{0}$, since for any feasible point $(\tilde{\boldsymbol{D}}, \boldsymbol{z})$ satisfying constraints in $(8)$, let us define $\Delta_{i}=-l_{i} z_{i}$ for each $i \in[n]$, then $(\tilde{\boldsymbol{D}}, \boldsymbol{\Delta})$ satisfies the constraints in $(3)$. Indeed, we can show that

Proposition 1. R-MNMS (7) is equivalent to

$$
v^{*}=\max _{\boldsymbol{Q} \in \mathbb{R}_{+}^{n}} \min _{\tilde{\boldsymbol{D}} \in \mathcal{U}} \Pi(\boldsymbol{Q}, \tilde{\boldsymbol{D}}),
$$

where $\mathcal{U}$ is defined in (8).

Proof. Let $v_{1}$ denote the optimal value of Model (9), then we only need to show $v_{1}=v^{*}$.

(i) $v_{1} \geq v^{*}$. For any $\tilde{\boldsymbol{D}} \in \mathcal{U}_{0}$, there exists $\boldsymbol{\Delta}$ such that $\|\boldsymbol{\Delta}\|_{0} \leq k, \tilde{D}_{i}=D_{i}+\Delta_{i},-l_{i} \leq \Delta_{i} \leq u_{i}$. Let us define binary variable $z_{i}=\left\{\begin{array}{ll}0, & \text { if } \Delta_{i}=0 \\ 1, & \text { if } \Delta_{i} \neq 0\end{array}\right.$ for each $i \in[n]$. Since $\|\boldsymbol{\Delta}\|_{0} \leq k$, thus we must have $\sum_{i \in[n]} z_{i} \leq k$. Let us define $\tilde{D}_{i}^{*}=D_{i}-l_{i} z_{i}$ for each $i \in[n]$. Clearly, we have $\tilde{\boldsymbol{D}}^{*} \in \mathcal{U}$ and $\tilde{\boldsymbol{D}}^{*} \leq \tilde{\boldsymbol{D}}$. For any fixed $\boldsymbol{Q} \in \mathbb{R}_{+}^{n}$, by Lemma 1 , we know that the profit function $\Pi(\boldsymbol{Q}, \tilde{\boldsymbol{D}})$ is nondecreasing in the demand $\tilde{\boldsymbol{D}}$. Thus, $\Pi(\tilde{Q}, \tilde{\boldsymbol{D}}) \geq \Pi\left(\boldsymbol{Q}, \tilde{\boldsymbol{D}}^{*}\right)$, which implies $\min _{\tilde{\boldsymbol{D}} \in \mathcal{U}_{0}} \Pi(\boldsymbol{Q}, \tilde{\boldsymbol{D}}) \geq \min _{\tilde{\boldsymbol{D}} \in \mathcal{U}} \Pi(\boldsymbol{Q}, \tilde{\boldsymbol{D}})$ for any $\boldsymbol{Q} \in \mathbb{R}_{+}^{n}$. This proves $v_{1} \geq v^{*}$. 
(ii) $v_{1} \leq v^{*}$. Since $\mathcal{U}_{0} \supseteq \mathcal{U}$, thus for any $\boldsymbol{Q} \in \mathbb{R}_{+}^{n}, \min _{\tilde{\boldsymbol{D}} \in \mathcal{U}_{0}} \Pi(\boldsymbol{Q}, \tilde{\boldsymbol{D}}) \leq \min _{\tilde{\boldsymbol{D}} \in \mathcal{U}} \Pi(\boldsymbol{Q}, \tilde{\boldsymbol{D}})$, thus, $v_{1} \leq v^{*}$.

From Proposition 1, by substituting $\tilde{D}_{i}=D_{i}-l_{i} z_{i}$ in (6) and defining the following cardinality set

$$
X=\left\{\boldsymbol{z}: \sum_{i \in[n]} z_{i} \leq k, z_{i} \in\{0,1\}\right\}
$$

then we can have the following equivalent formulation of R-MNMS:

$$
v^{*}=\max _{\boldsymbol{Q} \in \mathbb{R}_{+}^{n}}\left\{f(\boldsymbol{Q}):=\sum_{i \in[n]} \bar{P}_{i} Q_{i}-R(\boldsymbol{Q})\right\}
$$

where

$$
R(\boldsymbol{Q}):=\max _{\boldsymbol{z} \in X} \sum_{i \in[n]} \bar{S}_{i}\left(Q_{i}-D_{i}+l_{i} z_{i}-\sum_{j \in[n]} \underline{\alpha}_{j i}\left(D_{j}-l_{j} z_{j}-Q_{j}\right)_{+}\right)_{+} .
$$

This new equivalent formulation (11) allows us to compute the worst-case profit function via an integer program rather than a nonconvex program, which can be further reduced to a MILP in Section 5 ,

One direct benefit of formulation 11 is that we can easily derive upper bounds of optimal order quantities. The result can be proved by contradiction.

Proposition 2. There exists an optimal solution $\boldsymbol{Q}^{*}$ to $R$-MNMS such that for each product $i \in[n]$, $Q_{i}^{*} \leq M_{i}$, where $M_{i}=D_{i}+\sum_{j \in[n]} \underline{\alpha}_{j i} D_{j}$.

Proof. See Appendix A.1.

This result is very useful to derive an equivalent MILP formulation of R-MNMS in Section 5 . Remark 2. If Assumption 1 does not hold (i.e., $k^{\alpha} \leq n^{2}-n$ ) and $k=n$, then similar to formulation (11), we can have the following equivalent formulation of $R$-MNMS

$$
v^{*}=\max _{\boldsymbol{Q} \in \mathbb{R}_{+}^{n}}\left\{f(\boldsymbol{Q}):=\sum_{i \in[n]} \bar{P}_{i} Q_{i}-R(\boldsymbol{Q})\right\}
$$

where

$$
R(\boldsymbol{Q}):=\max _{\boldsymbol{z} \in X^{\alpha}} \sum_{i \in[n]} \bar{S}_{i}\left(Q_{i}-\underline{D}_{i}-\sum_{j \in[n]}\left(\tilde{\alpha}_{j i}-l_{j i}^{\alpha} z_{j i}^{\alpha}\right)\left(\underline{D}_{j}-Q_{j}\right)_{+}\right)_{+},
$$

where $X^{\alpha}=\left\{z^{\alpha}: \sum_{j \in[n]} \sum_{i \in[n]} z_{j i}^{\alpha} \leq k^{\alpha}, z_{j i}^{\alpha} \in\{0,1\}\right\}$. Also, we will have $M_{i}=\underline{D}_{i}+\sum_{j \in[n]} \tilde{\alpha}_{j i} \underline{D}_{j}$. 


\subsection{Complexity of the Inner Maximization Problem (11b)}

It has been shown in Zhang et al. (2018) that even if $k=0, k^{\alpha}=0$, solving R-MNMS can be NP-hard. In this subsection, we will show that the inner maximization problem (11b) of R-MNMS is also NP-hard.

First, observe that

$$
\left(D_{j}-l_{j} z_{j}-Q_{j}\right)_{+}= \begin{cases}\left(D_{j}-l_{j}-Q_{j}\right)_{+}, & \text {if } z_{j}=1 \\ \left(D_{j}-Q_{j}\right)_{+}, & \text {if } z_{j}=0\end{cases}
$$

for each $j \in[n]$, thus this observation allows us to linearize nonlinear expressions $\left\{\left(D_{j}-l_{j} z_{j}\right.\right.$ $\left.\left.-Q_{j}\right)_{+}\right\}_{j \in[n]}$ and to rewrite $11 \mathrm{~b}$ as

$$
R(\boldsymbol{Q})=\max _{\boldsymbol{z} \in X}\left\{\sum_{i \in[n]} \bar{S}_{i}\left[Q_{i}-D_{i}+l_{i} z_{i}-\sum_{j \in[n]} \underline{\alpha}_{j i}\left(\left(D_{j}-l_{j}-Q_{j}\right)_{+} z_{j}+\left(D_{j}-Q_{j}\right)_{+}\left(1-z_{j}\right)\right)\right]_{+}\right\} .
$$

Next, we show that the inner maximization problem (13) is NP-hard via a reduction to the well known clique problem.

Theorem 1. The inner maximization problem (13) in general is NP-hard.

Proof. See Appendix A.2.

Theorem 1 shows that unlike many robust optimization problems, it might be difficult to derive a tractable form for the general inner maximization problem (13). Thus, instead, in Section 4 , we propose three special cases such that both inner maximization (13) and R-MNMS are tractable. For general R-MNMS, we propose an equivalent MILP reformulation and develop exact and approximate algorithms to solve it, which will be presented in Section 5 .

\section{Three Special Cases: Closed-form Optimal Solutions}

In this section, we will study three different special cases of R-MNMS (11) and derive their closed-form optimal solutions.

\subsection{Special Case I: $n=2, k=1$}

In this section, we study R-MNMS with only two products (i.e., $n=2$ ) and the budget of uncertainty is equal to 1 (i.e., $k=1$ in set $X$ defined in (10)). Note that if $k=0$ or 2 , it reduces to Special Case III, which will be discussed in Section 4.3. Under this setting, R-MNMS (11) becomes:

$$
v^{*}=\max _{\boldsymbol{Q} \in \mathbb{R}_{+}^{2}}\left\{\sum_{i \in[2]} \bar{P}_{i} Q_{i}-\max _{\boldsymbol{z} \in X} \sum_{i \in[2]} \bar{S}_{i}\left(Q_{i}-D_{i}+l_{i} z_{i}-\sum_{j \in[2]} \underline{\alpha}_{j i}\left(D_{j}-l_{j} z_{j}-Q_{j}\right)_{+}\right)_{+}\right\},
$$


and $X=\left\{\boldsymbol{z}: z_{1}+z_{2} \leq 1, z_{i} \in\{0,1\}, \forall i \in[2]\right\}$. To simplify our closed-form solutions, we further make the following assumption.

Assumption 2. Suppose that $D_{2} \underline{\alpha}_{21} \geq l_{1} \geq \underline{\alpha}_{21} l_{2}, D_{1} \underline{\alpha}_{12} \geq l_{2} \geq \underline{\alpha}_{12} l_{1}$.

Assumption 2 postulates that the demand deviation of one product cannot be smaller than the substitution part of the other product's demand deviation and cannot be larger than the substitution part of the other product's nominal demand. Please note that our analysis is general and can be also applied to the other parametric settings without satisfying Assumption 2. However, for the purpose of brevity, we will stick to this assumption in this subsection.

The next theorem presents our main findings of the optimal order quantities for this special case under Assumption 2. These key findings are: (i) to divide the feasible regions into 9 subregions by comparing $Q_{i}$ with $D_{i}-l_{i}$ and $D_{i}$ for each $i \in$ [2]; (ii) for each subregion, R-MNMS (14) becomes a concave maximization problem with a piecewise linear objective function. Thus one of its optimal solutions can be achieved by an extreme point; and (iii) for each subregion, there are few potential optimal solutions. Thus, we enumerate all the candidate solutions and find the one which achieves the highest total profit across all 9 subregions.

Theorem 2. Suppose $n=2, k=1$, and Assumption 2 holds, then the optimal order quantities $\boldsymbol{Q}^{*}=\left(Q_{1}^{*}, Q_{2}^{*}\right)$ are characterized by the following three cases:

Case 1: If $\bar{P}_{1} \leq \bar{P}_{2} \underline{\alpha}_{12}$ and $\bar{P}_{2} \geq \bar{P}_{1} \underline{\alpha}_{21}$, then $\left(Q_{1}^{*}, Q_{2}^{*}\right)=\left(0, D_{2}-l_{2}+\underline{\alpha}_{12} D_{1}\right)$.

Case 2: If $\bar{P}_{2} \leq \bar{P}_{1} \underline{\alpha}_{21}$ and $\bar{P}_{1} \geq \bar{P}_{2} \underline{\alpha}_{12}$, then $\left(Q_{1}^{*}, Q_{2}^{*}\right)=\left(D_{1}-l_{1}+\underline{\alpha}_{21} D_{2}, 0\right)$.

Case 3: If $\bar{P}_{1} \geq \bar{P}_{2} \underline{\alpha}_{12}$ and $\bar{P}_{2} \geq \bar{P}_{1} \underline{\alpha}_{21}$, then we have the following two sub-cases:

Sub-case 3.1: If $\bar{S}_{1} l_{1} \geq \bar{S}_{2} l_{2}$, then $\left(Q_{1}^{*}, Q_{2}^{*}\right)=\left(D_{1}-\frac{l_{1}-\underline{\alpha}_{21} l_{2}}{1-\underline{\alpha}_{12} \underline{\alpha}_{21}}, D_{2}-\frac{l_{2}-\underline{\alpha}_{12} l_{1}}{1-\underline{\alpha}_{12} \underline{\alpha}_{21}}\right)$ or $\left(Q_{1}^{*}, Q_{2}^{*}\right)=$ $\left(D_{1}, D_{2}-\frac{\bar{S}_{2} l_{2}-\bar{S}_{1} l_{1}}{\bar{S}_{2}-\bar{S}_{1} \underline{\alpha}_{21}}\right)$.

Sub-case 3.2: If $\bar{S}_{1} l_{1} \leq \bar{S}_{2} l_{2}$, then $\left(Q_{1}^{*}, Q_{2}^{*}\right)=\left(D_{1}-\frac{l_{1}-\underline{\alpha}_{21} l_{2}}{1-\underline{\alpha}_{12} \underline{\alpha}_{21}}, D_{2}-\frac{l_{2}-\underline{\alpha}_{12} l_{1}}{1-\underline{\alpha}_{12} \underline{\alpha}_{21}}\right)$ or $\left(Q_{1}^{*}, Q_{2}^{*}\right)=$ $\left(D_{1}-\frac{\bar{S}_{1} l_{1}-\bar{S}_{2} l_{2}}{\bar{S}_{1}-\bar{S}_{2} \underline{\alpha}_{12}}, D_{2}\right)$.

Proof. See Appendix A.3.

Theorem 2 provides a complete characterization of optimal order quantities of the two-product case, which highly depend on the comparison between the marginal profit of product $i$ and the profit generated by using product $j$ to substitute product $i$. In particular, we make the following remarks.

Remark 3. (i) In Case 1, suppose that the marginal profit of product 1 is lower than the profit generated by using product 2 to substitute product 1 , but the marginal profit of product 2 is higher than the profit generated by using product 1 to substitute product 2 , i.e., product 2 is 
much more profitable than product 1 . In this case, the retailer should only order product 2 to satisfy their customers' demand and satisfy part of the customers' demand for product 1 by substitution. In this case, the worst-case demand of product 2 is $D_{2}-l_{2}$ while the worst-case demand of product 1 is equal to the nominal demand $D_{1}$.

(ii) The interpretation of Case 2 is similar and thus is omitted for brevity.

(iii) In Case 3, if the marginal profit of one product is higher than the profit generated by using the other product to substitute this product (i.e., both products are similarly profitable), then the optimal order quantities depend on the relationship between $\bar{S}_{1} l_{1}$ and $\bar{S}_{2} l_{2}$. One special case is that when $s_{i}=c_{i}$ for each product $i \in[2]$, i.e., the salvage value of each product is equal to its unit production cost, the optimal order quantity of product 1 is $Q_{1}^{*}=D_{1}-\frac{l_{1}-\underline{\alpha}_{21} l_{2}}{1-\underline{\alpha}_{12} \underline{\alpha}_{21}}$ and the optimal order quantity of product 2 is $Q_{2}^{*}=D_{2}-\frac{l_{2}-\underline{\alpha}_{12} l_{1}}{1-\underline{\alpha}_{12} \underline{\alpha}_{21}}$, while the worst-case demand of products 1 and 2 can be $\left(D_{1}, D_{2}-l_{2}\right)$ or $\left(D_{1}-l_{1}, D_{2}\right)$, respectively. If there is a tie between two solutions in Sub-case 3.1 or Sub-case 3.2, then one can randomly pick one solution as both of them are optimal.

(iv) It is impossible that $\bar{P}_{1}<\bar{P}_{2} \underline{\alpha}_{12}, \bar{P}_{2}<\bar{P}_{1} \underline{\alpha}_{21}$, which implies $1<\underline{\alpha}_{12} \underline{\alpha}_{21}$, contradicting the assumption that all the substitution rates are between 0 and 1 .

\subsection{Special Case II: $\underline{\boldsymbol{\alpha}}=\mathbf{0}$}

In this subsection, we analyze robust multi-product newsvendor problem without substitution, i.e., $\underline{\boldsymbol{\alpha}}=\mathbf{0}$. In this setting, the effective demand becomes $\tilde{D}_{i}^{s}(\boldsymbol{Q})=\tilde{D}_{i}=D_{i}-l_{i} z_{i}$. Thus, R-MNMS (11) reduces to:

$$
v^{*}=\max _{\boldsymbol{Q} \in \mathbb{R}_{+}^{n}}\left\{f(\boldsymbol{Q}):=\sum_{i \in[n]} \bar{P}_{i} Q_{i}-\max _{\boldsymbol{z} \in X} \sum_{i \in[n]} \bar{S}_{i}\left(Q_{i}-D_{i}+l_{i} z_{i}\right)_{+}\right\},
$$

where set $X$ is defined in $(10)$. We first make the following observation.

Lemma 2. There exists an optimal solution $Q^{*}$ of Model (15) such that $D_{i}-l_{i} \leq Q_{i}^{*} \leq D_{i}$ for all $i \in[n]$.

Proof. For notational convenience, let us define $\boldsymbol{Q}_{-i}=\left[Q_{1}, \cdots, Q_{i-1}, Q_{i+1}, \cdots, Q_{n}\right]^{\top}$ to be the vector of the remaining elements of $\boldsymbol{Q}$. It is sufficient to show that for any fixed $\boldsymbol{Q}_{-i} \in \mathbb{R}_{+}^{n-1}$, the objective function of Model (15), $f\left(Q_{i}, \boldsymbol{Q}_{-i}\right)$, is monotone nondecreasing in $Q_{i}$ when $Q_{i} \in\left[0, D_{i}-l_{i}\right]$ and monotone nonincreasing in $Q_{i}$ when $Q_{i} \in\left[D_{i},+\infty\right)$. Indeed, we note that

$$
\begin{aligned}
& f\left(Q_{i}, \boldsymbol{Q}_{-i}\right) \\
= & \sum_{\tau \in[n] \backslash\{i\}} \bar{P}_{\tau} Q_{\tau}+\bar{P}_{i} Q_{i}-\max _{z \in X}\left(\sum_{\tau \in[n] \backslash\{i\}} \bar{S}_{\tau}\left(Q_{\tau}-D_{\tau}+l_{\tau} z_{\tau}\right)_{+}+\bar{S}_{i}\left(Q_{i}-D_{i}+l_{i} z_{i}\right)_{+}\right)
\end{aligned}
$$




$$
= \begin{cases}\sum_{\tau \in[n] \backslash\{i\}} \bar{P}_{\tau} Q_{\tau}-\max _{z \in X} \sum_{\tau \in[n] \backslash\{i\}} \bar{S}_{\tau}\left(Q_{\tau}-D_{\tau}+l_{\tau} z_{\tau}\right)_{+}+\bar{P}_{i} Q_{i}, & \text { if } Q_{i} \in\left[0, D_{i}-l_{i}\right], \\ \sum_{\tau \in[n] \backslash\{i\}} \bar{P}_{\tau} Q_{\tau}-\max _{z \in X}\left(\sum_{\tau \in[n] \backslash\{i\}} \bar{S}_{\tau}\left(Q_{\tau}-D_{\tau}+l_{\tau} z_{\tau}\right)_{+}-D_{i}+l_{i} z_{i}\right)+\left(\bar{P}_{i}-\bar{S}_{i}\right) Q_{i}, & \text { if } Q_{i} \in\left[D_{i},+\infty\right) .\end{cases}
$$

Clearly, from the above equation, we know that if $Q_{i} \in\left[0, D_{i}-l_{i}\right]$, the coefficient of $Q_{i}$ is $\bar{P}_{i}$, which is nonnegative, while if $Q_{i} \in\left[D_{i},+\infty\right)$, the coefficient of $Q_{i}$ is $\bar{P}_{i}-\bar{S}_{i}$, which is nonpositive. Thus, $f\left(Q_{i}, Q_{-i}\right)$ is nondecreasing on $Q_{i}$ when $Q_{i} \in\left[0, D_{i}-l_{i}\right]$ and nonincreasing on $Q_{i}$ when $Q_{i} \in\left[D_{i},+\infty\right)$. This completes the proof.

According to Lemma 2, without loss of generality, we can assume in Model (15), $\boldsymbol{Q} \in[\boldsymbol{D}-$ $\boldsymbol{l}, \boldsymbol{D}]$. Thus, for each $i \in[n],\left(Q_{i}-D_{i}+l_{i} z_{i}\right)_{+}=\left\{\begin{array}{ll}0, & \text { if } z_{i}=0 \\ Q_{i}-D_{i}+l_{i}, & \text { if } z_{i}=1\end{array}=\left(Q_{i}-D_{i}+l_{i}\right) z_{i}\right.$. Therefore, Model (15) is equivalent to

$$
v^{*}=\max _{\boldsymbol{Q} \in[\boldsymbol{D}-\boldsymbol{l}, \boldsymbol{D}]}\left(\sum_{i \in[n]} \bar{P}_{i} Q_{i}-\max _{\boldsymbol{z} \in X} \sum_{i \in[n]} \bar{S}_{i}\left(Q_{i}-D_{i}+l_{i}\right) z_{i}\right),
$$

where $X$ is defined in 10 .

Suppose that $\{(1),(2), \cdots,(n)\}$ is a permutation of $[n]$ such that $\bar{S}_{(1)} l_{(1)} \geq \bar{S}_{(2)} l_{(2)} \geq \cdots \geq$ $\bar{S}_{(n)} l_{(n)}$. We can obtain a closed-form optimal solution to Model (16) as follows.

Theorem 3. When $\underline{\boldsymbol{\alpha}}=0$, the optimal solutions $\boldsymbol{Q}^{*}$ of Model (16) are characterized as follows:

(i) If $\sum_{i \in[n]} \bar{P}_{i} \bar{S}_{i} \leq k$, then $Q_{i}^{*}=D_{i}-l_{i}$, and $v^{*}=\sum_{i \in[n]} \bar{P}_{i}\left(D_{i}-l_{i}\right)$.

(ii) If $\sum_{i \in[n]} \frac{\bar{P}_{i}}{\bar{S}_{i}}>k$,

$$
Q_{i}^{*}= \begin{cases}D_{i}-l_{i}+\frac{\bar{S}_{(t+1)} l_{(t+1)},}{\bar{S}_{i}}, & \text { if } i \in T \\ D_{i}, & \text { if } i \in[n] \backslash T\end{cases}
$$

and

$$
v^{*}=\sum_{i \in[n] \backslash T} \bar{P}_{i} l_{i}-\bar{S}_{(t+1)} l_{(t+1)} k+\sum_{i \in T} \frac{\bar{P}_{i}}{\bar{S}_{i}} \bar{S}_{(t+1)} l_{(t+1)}+\sum_{i \in[n]} \bar{P}_{i}\left(D_{i}-l_{i}\right),
$$

where set $T:=\{(1),(2), \cdots,(t)\}$ satisfying $\sum_{i \in T} \frac{\bar{P}_{i}}{\bar{S}_{i}} \leq k, \sum_{i \in T \cup\{(t+1)\}} \frac{\bar{P}_{i}}{\bar{S}_{i}}>k$.

Proof. See Appendix A.4.

Theorem 3 reveals the impact of the budget of uncertainty on optimal order quantities. Indeed, if $\sum_{i \in[n]} \frac{\bar{P}_{i}}{\bar{S}_{i}} \leq k$, i.e., the budget of uncertainty $k$ is no smaller than the sum of the critical ratios of all the products, then in this case, the optimal order quantity for each product is equal to the 
lower bound of the demand, i.e., $Q_{i}^{*}=D_{i}-l_{i}$ for each $i \in[n]$. Hence, this implies that when the products are not very profitable, or the accuracy of demand forecasting is relatively low, then the decision of the retailer should be conservative to hedge against unnecessary loss from demand forecasting. Suppose that $\sum_{i \in[n]} \bar{P}_{i} \bar{S}_{i}>k$, i.e., the budget of uncertainty is smaller than the sum of critical ratios of all the products, or equivalently, relatively a small amount of demand can be allowed to deviate from the nominal demand $\boldsymbol{D}$. Also, note that for each product $i \in[n]$, the value of $\bar{S}_{i} l_{i}$ can be interpreted as the risk of lost sales for product $i$ when its order quantity is $D_{i}$ with the worst-case demand $D_{i}-l_{i}$ (i.e., the sum of underage cost and overage cost multiplies the demand deviation). In this case, for each product $i \in T$ whose risk of lost sales is larger than a threshold $\bar{S}_{(t+1)} l_{(t+1)}$, its order quantities should be equal to $D_{i}-l_{i}+\frac{\bar{S}_{(t+1)} l_{(t+1)}}{\bar{S}_{i}}$; otherwise, it should be $D_{i}$. The threshold $\bar{S}_{(t+1)} l_{(t+1)}$ can be determined by searching for the product such that the sum of the critical ratios of the products whose risk is higher than product $(t+1)$ is no larger than the budget of uncertainty $k$, but including the critical ratio of this product into the sum will be above $k$. This result indicates that the products with a lower risk of lost sales should be ordered up to the nominal demand, while those with higher risk should be ordered less than the nominal demand.

\subsection{Special Case III: $k=n$}

When the budget of uncertainty is equal to $n$, i.e., $k=n$, the uncertainty set $\mathcal{U}$ becomes

$$
\mathcal{U}=\left\{\tilde{\boldsymbol{D}}: \tilde{D}_{i}=D_{i}-l_{i} z_{i}, \sum_{i \in[n]} z_{i} \leq n, z_{i} \in\{0,1\}, \forall i \in[n]\right\} .
$$

From Lemma 1, we know that the profit function $\Pi(\boldsymbol{Q}, \tilde{\boldsymbol{D}})$ is nonincreasing in $\tilde{\boldsymbol{D}}$, thus at the optimality, we must have $z_{i}=1$ for all $i \in[n]$ in the inner maximization problem (11b), i.e., the worst-case demand in this special case will always be equal to $\boldsymbol{D}-\boldsymbol{l}$. Then, Model (11) becomes

$$
v^{*}=\max _{Q \in \mathbb{R}_{+}^{n}} \sum_{i \in[n]} \bar{P}_{i} Q_{i}-\sum_{i \in[n]} \bar{S}_{i}\left[Q_{i}-D_{i}+l_{i}-\sum_{j \in[n]} \underline{\alpha}_{j i}\left(D_{j}-l_{j}-Q_{j}\right)_{+}\right]_{+} .
$$

Note that Model (17) is a multi-product newsvendor model with substitution when the demand is deterministic and is equal to $\boldsymbol{D}-\boldsymbol{l}$. According to the recent work in Zhang et al. (2018), the optimal order quantities of Model (17) can be completely characterized as follows (For more details, please refer to Zhang et al. (2018)).

Theorem 4. (Theorem 1, Zhang et al. (2018)) When $k=n$, the optimal order quantities $\boldsymbol{Q}^{*}$ and the optimal total profit $v^{*}$ are characterized as follows: 
(i)

$$
Q_{j}^{*}= \begin{cases}D_{j}^{s}\left(\boldsymbol{Q}^{*}\right)=D_{j}-l_{j}+\sum_{i \in \Gamma^{*}} \underline{\alpha}_{i j}\left(D_{i}-l_{i}\right), & \text { if } \bar{P}_{j}-\sum_{i \in[n] \backslash \Gamma^{*}} \underline{\alpha}_{j i} \bar{P}_{i} \geq 0 \\ 0, & \text { otherwise }\end{cases}
$$

for each $j \in[n]$, where $[n] \backslash \operatorname{supp}\left(\boldsymbol{Q}^{*}\right)=\Gamma^{*}$, i.e., $\Gamma^{*}=\left\{i \in[n]: Q_{i}^{*}=0\right\}$; and

(ii)

$$
v^{*}=\max _{\Gamma \subseteq[n]}\left\{f(\Gamma):=\sum_{j \in \Gamma \in[n] \backslash \Gamma} \sum_{j i} \bar{\alpha}_{i}\left(D_{j}-l_{j}\right)+\sum_{i \in[n] \backslash \Gamma} \bar{P}_{i}\left(D_{i}-l_{i}\right)\right\}:=f\left(\Gamma^{*}\right),
$$

In Theorem 4, if the budget of uncertainty is equal to the number of products, then for each product $j \in[n]$, its optimal order quantity $\boldsymbol{Q}_{j}^{*}$ is equal to its effective demand if its marginal profit $\bar{P}_{j}$ is larger than or equal to the sum of the profits generated by using other products to substitute it, and 0 , otherwise. This suggests that the retailer does not need to order a product if its marginal profit is relatively low and should order up to its effective demand, otherwise. Also, in $(19)$, the first term is the sum of the total profit for selling product $i \in[n] \backslash \Gamma$ to meet the demand of its substitutable products $j \in \Gamma$ and the second term is the profit of selling product $i \in[n] \backslash \Gamma$ to meet its own demand. Finally, please note that although we completely characterize the optimal order quantities for all the products, obtaining these values is in general NP-hard (Zhang et al., 2018).

Another interesting observation from Theorem 4 is that the optimal order quantity for each product can be equal to their worst-case demand, i.e., $Q_{j}^{*}=D_{j}-l_{j}$ for each product $j \in[n]$, under the following assumptions.

Corollary 1. Suppose (1) $\bar{P}_{i}=\bar{P}_{j}, \forall i, j \in[n]$ and (2) for each product $j \in[n], \sum_{i \in[n]} \underline{\alpha}_{j i}<1$. Then $Q_{j}^{*}=D_{j}-l_{j}$ for all $j \in[n]$.

Proof. Note that from Theorem 4, the optimal subset $\Gamma^{*}=\emptyset$. Therefore, $Q_{j}^{*}=D_{j}^{s}\left(\boldsymbol{Q}^{*}\right)=D_{j}-l_{j}$ for all $j \in[n]$.

Corollary 1 shows that if all the products share the same underage cost and cannot be completely substituted by the others, then the optimal order quantities are equal to the worst-case demand, i.e., $Q_{j}^{*}=D_{j}-l_{j}$ for each product $j \in[n]$.

Finally, we remark that if $k=0$, then the results in Theorem 4 will also hold simply by replacing $l_{i}=0$ for each $i \in[n]$.

\section{Solution Approaches}

Note that the inner maximization Model $(11 \mathrm{~b})$ is a nonconvex and nonsmooth optimization problem. In this section, we will introduce equivalent MILP formulations for R-MNMS (11) and 
its inner maximization Model (11b) by linearizing the nonconvex terms in the profit function. These equivalent formulations allow us to develop an effective branch and cut algorithm and an alternative conservative approximation to solve R-MNMS.

\subsection{An Equivalent MILP Formulation of the Inner Maximization Problem}

In this subsection, we will present an MILP formulation, which is equivalent to the inner maximization problem $11 \mathrm{~b}$. To begin with, in 11b), let us define two new variables

$$
u_{j}=\left(D_{j}-l_{j}-Q_{j}\right)_{+}, \quad \psi_{j}=\left(D_{j}-Q_{j}\right)_{+}
$$

for each $j \in[n]$. Clearly, we have $\psi_{j} \geq u_{j}$ for each $j \in[n]$. For simplicity, we still use the function $R(\boldsymbol{Q}, \boldsymbol{u}, \boldsymbol{\psi})$ to denote the optimal value of inner maximization problem $11 \mathrm{~b}$ for any given $\boldsymbol{Q}, \boldsymbol{u}, \boldsymbol{\psi}$, i.e., the inner maximization problem becomes

$$
\begin{aligned}
R(\boldsymbol{Q}, \boldsymbol{u}, \boldsymbol{\psi})=\max _{\boldsymbol{z}} \sum_{i \in[n]} \bar{S}_{i}\left[Q_{i}-D_{i}+l_{i} z_{i}-\sum_{j \in[n]} \underline{\alpha}_{j i}\left(u_{j} z_{j}+\psi_{j}\left(1-z_{j}\right)\right)\right]_{+}, \\
\text {s.t. } \sum_{i \in[n]} z_{i} \leq k, \\
\quad z_{i} \in\{0,1\}, \forall i \in[n] .
\end{aligned}
$$

Note that Model (20) is a convex integer maximization problem. Thus, we will further linearize the objective function into a linear form. To do so, for each $i \in[n]$, let us define a binary variable $x_{i}=1$, if $Q_{i}-D_{i}+l_{i} z_{i}-\sum_{j} \underline{\alpha}_{j i}\left(u_{j} z_{j}+\psi_{j}\left(1-z_{j}\right)\right) \geq 0$, and 0, otherwise. Thus, Model 20 is equivalent to

$$
\begin{aligned}
R(\boldsymbol{Q}, \boldsymbol{u}, \boldsymbol{\psi})=\max _{\boldsymbol{x}, \boldsymbol{z}} \sum_{i \in[n]} \bar{S}_{i}\left[Q_{i}-D_{i}+l_{i} z_{i}-\sum_{j \in[n]} \underline{\alpha}_{j i}\left(u_{j} z_{j}+\psi_{j}\left(1-z_{j}\right)\right)\right] x_{i}, \\
\text { s.t. } \sum_{i \in[n]} z_{i} \leq k \\
\quad x_{i}, z_{i} \in\{0,1\}, \forall i \in[n] .
\end{aligned}
$$

The above Model (21) now becomes a binary bilinear program, which can be further linearized by introducing new variables representing the bilinear terms. The final reformulation result is shown below.

\footnotetext{
${ }^{1}$ For the general $k^{\alpha}$, we can derive the similar MILP formulation, which can be found in Appendix B
} 
Proposition 3. The inner maximization problem (20) is equivalent to

$$
\begin{aligned}
& R(\boldsymbol{Q}, \boldsymbol{u}, \boldsymbol{\psi})=\max _{\boldsymbol{x}, \boldsymbol{y}, \boldsymbol{z}} \sum_{i \in[n]} \bar{S}_{i}\left[\left(Q_{i}-D_{i}\right) x_{i}+l_{i} y_{i i}-\sum_{j \in[n]} \underline{\alpha}_{j i}\left(u_{j} y_{j i}+\psi_{j}\left(x_{i}-y_{j i}\right)\right)\right] \\
& \text { s.t. } \sum_{i \in[n]} z_{i} \leq k . \\
& \quad y_{j i} \leq x_{i}, \forall i, j \in[n], \\
& y_{j i} \leq z_{j}, \forall i, j \in[n], \\
& z_{i}, x_{i} \in\{0,1\}, y_{j i} \geq 0, \forall i, j \in[n] .
\end{aligned}
$$

Proof. See Appendix A.5.

\subsection{Reformulation of R-MNMS and branch and cut algorithm}

Next we are going to investigate an MILP reformulation for R-MNMS (11), which is amenable for a branch and cut algorithm. First, from Proposition 2, without loss of generality, we can assume that the order quantities $\boldsymbol{Q}$ can be upper bounded by $\boldsymbol{M}$. Thus, for each product $i \in[n]$, its order quantity $Q_{i}$ must belong to one of the following three intervals: $\left[0, D_{i}-l_{i}\right],\left[D_{i}-l_{i}, D_{i}\right],\left[D_{i}, M_{i}\right]$ (we break the boundary points arbitrarily). For notational convenience, let us denote $D_{i}^{(0)}=0$, $D_{i}^{(1)}=D_{i}-l_{i}, D_{i}^{(2)}=D_{i}$, and $D_{i}^{(3)}=M_{i}$. Next, we introduce one binary variable for each interval to indicate whether $Q_{i}$ is in this interval or not, i.e., we let $\chi_{i}^{(e)}=1$ if $Q_{i} \in\left[D_{i}^{(e-1)}, D_{i}^{(e)}\right]$ for each $e \in[3]$; and 0, otherwise. And we let

$$
\sum_{e \in[3]} \chi_{i}^{(e)}=1
$$

to enforce that $Q_{i}$ indeed belongs to only one interval. Correspondingly, for each product $i \in[n]$ and $e \in[3]$, we further introduce another variable $w_{i}^{(e)}$ to be equal to $Q_{i}$ if $Q_{i} \in\left[D_{i}^{(e-1)}, D_{i}^{(e)}\right]$, and 0 , otherwise. That is,

$$
\begin{aligned}
& D_{i}^{(e-1)} \chi_{i}^{(e)} \leq w_{i}^{(e)} \leq D_{i}^{(e)} \chi_{i}^{(e)}, \forall i \in[n], e \in[3], \\
& \sum_{e \in[3]} w_{i}^{(e)}=Q_{i}, \forall i \in[n] .
\end{aligned}
$$

Next, we can express $u_{i}$ and $\psi_{i}$ (recall that $u_{i}=\left(D_{i}-l_{i}-Q_{i}\right)_{+}$and $\left.\psi_{i}=\left(D_{i}-Q_{i}\right)_{+}\right)$as linear functions of variables $\left\{\chi_{i}^{(e)}\right\}_{e \in[2]}$ and $\left\{w_{i}^{(e)}\right\}_{e \in[2]}$ for each product $i \in[n]$, i.e.,

$$
\begin{gathered}
u_{i}=\left(D_{i}-l_{i}\right) \chi_{i}^{(1)}-w_{i}^{(1)}, \forall i \in[n], \\
\psi_{i}=D_{i} \sum_{e \in[2]} \chi_{i}^{(e)}-\sum_{e \in[2]} w_{i}^{(e)}, \forall i \in[n],
\end{gathered}
$$


Clearly, in (23d), if $Q_{i}>D_{i}-l_{i}$, then $u_{i}$ is equal to 0 since both $\chi_{i}^{(1)}=0, w_{i}^{(1)}=0$ and otherwise, it is equal to $D_{i}-l_{i}-Q_{i}$. And in (23e), if $Q_{i}>D_{i}$, then $\psi_{i}$ is equal to 0 since $\chi_{i}^{(1)}=\chi_{i}^{(2)}=$ $0, w_{i}^{(1)}=w_{i}^{(2)}=0$, and otherwise, it is equal to $D_{i}-Q_{i}$. For the inner maximization problem 22, let us also define function $g(\boldsymbol{Q}, \boldsymbol{u}, \boldsymbol{\psi}, \boldsymbol{x}, \boldsymbol{y}, \boldsymbol{z})$ to be its objective function, i.e.,

$$
g(\boldsymbol{Q}, \boldsymbol{u}, \boldsymbol{\psi}, \boldsymbol{x}, \boldsymbol{y}, \boldsymbol{z})=\sum_{i \in[n]} \bar{S}_{i}\left[\left(Q_{i}-D_{i}\right) x_{i}+l_{i} y_{i i}-\sum_{j \in[n]} \underline{\alpha}_{j i}\left(u_{j} y_{j i}+\psi_{j}\left(x_{i}-y_{j i}\right)\right)\right]
$$

and set $\Xi$ to be its feasible region, i.e.,

$$
\Xi=\{(\boldsymbol{x}, \boldsymbol{y}, \boldsymbol{z}): 22 \mathrm{~b}-22 \mathrm{e}\}
$$

In view of the above development, we have the following equivalent MILP formulation of RMNMS (11):

$$
\begin{aligned}
v^{*}=\max _{\boldsymbol{Q}, \boldsymbol{u}, \boldsymbol{\psi}, \boldsymbol{\chi}, \boldsymbol{w}, \eta} & \sum_{i \in[n]} \bar{P}_{i} Q_{i}-\eta \\
\text { s.t. } & \eta \geq g(\boldsymbol{Q}, \boldsymbol{u}, \boldsymbol{\psi}, \boldsymbol{x}, \boldsymbol{y}, \boldsymbol{z}), \forall(\boldsymbol{x}, \boldsymbol{y}, \boldsymbol{z}) \in \Xi \\
& w_{i}^{(e)}, u_{i}, \psi_{i} \geq 0, \chi_{i}^{(e)} \in\{0,1\}, \forall i \in[n], e \in[3] . \\
& \text { 23a }-23 \mathrm{e} .
\end{aligned}
$$

Note that in (24b), there can be exponentially many constraints. Therefore, we propose a branch and cut algorithm to solve Model (24). To begin with, suppose we are given a subset $\widehat{\Xi} \subseteq \Xi$, which can be empty, then the master problem is formulated as below:

$$
\left.\left.\max _{\boldsymbol{Q}, \boldsymbol{u}, \boldsymbol{\psi}, \boldsymbol{\chi}, \boldsymbol{w}, \eta}\left\{\sum_{i \in[n]} \bar{P}_{i} Q_{i}-\eta: \eta \geq g(\boldsymbol{Q}, \boldsymbol{u}, \boldsymbol{\psi}, \boldsymbol{x}, \boldsymbol{y}, \boldsymbol{z}), \forall(\boldsymbol{x}, \boldsymbol{y}, \boldsymbol{z}) \in \widehat{\Xi}, 23 \mathrm{a}-23 \mathrm{e}\right), 24 \mathrm{c}\right)\right\} .
$$

Clearly, Model (25) is a relaxation of Model $(24)$, since $\widehat{\Xi} \subseteq \Xi$. Given an optimal solution $(\widehat{\boldsymbol{Q}}, \widehat{\boldsymbol{u}}, \widehat{\boldsymbol{\psi}}, \widehat{\boldsymbol{\chi}}, \widehat{\boldsymbol{w}}, \widehat{\eta})$ to the master problem 25 to check whether this solution is optimal to original Model (24) or not, it is sufficient to check whether it satisfies constraints (24b), i.e., solve the inner maximization problem $(22)$ by letting $(\boldsymbol{Q}, \boldsymbol{u}, \boldsymbol{\psi})=(\widehat{\boldsymbol{Q}}, \widehat{\boldsymbol{u}}, \widehat{\boldsymbol{\psi}})$ as below:

$$
R(\widehat{\boldsymbol{Q}}, \widehat{\boldsymbol{u}}, \widehat{\boldsymbol{\psi}})=\max _{(\boldsymbol{x}, \boldsymbol{y}, \boldsymbol{z}) \in \Xi}\{g(\widehat{\boldsymbol{Q}}, \widehat{\boldsymbol{u}}, \widehat{\boldsymbol{\psi}}, \boldsymbol{x}, \boldsymbol{y}, \boldsymbol{z})\}
$$

and check if $\widehat{\eta} \geq R(\widehat{\boldsymbol{Q}}, \widehat{\boldsymbol{u}}, \widehat{\boldsymbol{\psi}})$ or not. If $\widehat{\eta} \geq R(\widehat{\boldsymbol{Q}}, \widehat{\boldsymbol{u}}, \widehat{\boldsymbol{\psi}})$, then $(\widehat{\boldsymbol{Q}}, \widehat{\boldsymbol{u}}, \widehat{\boldsymbol{\psi}}, \widehat{\chi}, \widehat{\boldsymbol{w}}, \widehat{\eta})$ is optimal to Model 
(24). Otherwise, let $(\widehat{\boldsymbol{x}}, \widehat{\boldsymbol{y}}, \widehat{\boldsymbol{z}})$ be an optimal solution to Model (26). Then add a new constraint

$$
\eta \geq g(\boldsymbol{Q}, \boldsymbol{u}, \boldsymbol{\psi}, \widehat{\boldsymbol{x}}, \widehat{\boldsymbol{y}}, \widehat{\boldsymbol{z}})
$$

into the master problem (25) and continue. Note that this solution procedure can be integrated with branch and bound, which is known as "branch and cut" (Padberg and Rinaldi, 1991; Sen and Sherali, 2006, Bienstock and ÖZbay, 2008.

Below, we summarize the proposed branch and cut algorithm to solve Model (24), i.e., at each branch and bound node, we proceed the following cut generating procedure until achieving optimality.

Step 0: Initialize set $\widehat{\Xi}=\emptyset$.

Step 1: Solve the proposed master problem 25 with an optimal solution $(\widehat{\boldsymbol{Q}}, \widehat{\boldsymbol{u}}, \widehat{\boldsymbol{\psi}}, \widehat{\chi}, \widehat{\boldsymbol{w}}, \widehat{\eta})$.

Step 2: Solve Model (26), denote its optimal solution by $(\widehat{\boldsymbol{x}}, \widehat{\boldsymbol{y}}, \widehat{\boldsymbol{z}})$ and optimal value $R(\widehat{\boldsymbol{Q}}, \widehat{\boldsymbol{u}}, \widehat{\boldsymbol{\psi}})$.

Step 3: There are two cases:

Csse 1: If $\widehat{\eta} \geq R(\widehat{\boldsymbol{Q}}, \widehat{\boldsymbol{u}}, \widehat{\boldsymbol{\psi}})$, set $\boldsymbol{Q}^{*} \leftarrow \widehat{\boldsymbol{Q}}, \boldsymbol{u}^{*} \leftarrow \widehat{\boldsymbol{u}}, \boldsymbol{\psi}^{*} \leftarrow \widehat{\boldsymbol{\psi}}, \boldsymbol{\chi}^{*} \leftarrow \widehat{\boldsymbol{\chi}}, \boldsymbol{w}^{*} \leftarrow \widehat{\boldsymbol{w}}, \eta^{*} \leftarrow \widehat{\eta}$, stop and output the optimal solution $\left(\boldsymbol{Q}^{*}, \boldsymbol{u}^{*}, \boldsymbol{\psi}^{*}, \boldsymbol{\chi}^{*}, \boldsymbol{w}^{*}, \eta^{*}\right)$.

Csse 2: If $\widehat{\eta}<R(\widehat{\boldsymbol{Q}}, \widehat{\boldsymbol{u}}, \widehat{\boldsymbol{\psi}})$, then augment set $\widehat{\Xi}=\widehat{\Xi} \cup(\widehat{\boldsymbol{x}}, \widehat{\boldsymbol{y}}, \widehat{\boldsymbol{z}})$, and go to Step 1 .

Note that this branch and cut algorithm will terminate in a finite number of steps since there are only a finite number of points in set $\Xi$, as well as finite number of binary variables in the master problem. However, to generate a new constraint at Step 2 might be very time-consuming since it involves solving an MILP (26), i.e., the inner maximization problem 222). In the remaining part of this section, we will replace this MILP (26) by its continuous relaxation and derive a conservative approximation for R-MNMS.

\subsection{Conservative Approximation}

In practice, the branch and cut algorithm might not be efficient in solving very large-scale problem instances. In this section, we propose a simple but very effective conservative approximation to solve R-MNMS 24, i.e., the optimal solution from conservative approximation is a feasible solution to R-MNMS (24). We also provide some sufficient conditions under which this conservative approximation yields an exact optimal solution to R-MNMS 24).

To derive the conservative approximation, we simply relax variables $(\boldsymbol{x}, \boldsymbol{y}, \boldsymbol{z})$ in set $\boldsymbol{\Xi}$ to be continuous in R-MNMS (24), then we can obtain the following lower bound, i.e., a conservative approximation to Model (24):

$$
\left.v^{C A}=\max _{\boldsymbol{Q}, \boldsymbol{u}, \boldsymbol{\psi}, \boldsymbol{\chi}, \boldsymbol{w}, \eta}\left\{\sum_{i \in[n]} \bar{P}_{i} Q_{i}-\eta: \eta \geq g(\boldsymbol{Q}, \boldsymbol{u}, \boldsymbol{\psi}, \boldsymbol{x}, \boldsymbol{y}, \boldsymbol{z}), \forall(\boldsymbol{x}, \boldsymbol{y}, \boldsymbol{z}) \in \Xi_{C}, 23 \mathrm{a}-23 \mathrm{e}, 24 \mathrm{c}\right]\right\},
$$

where $\Xi_{C}$ denotes the continuous relaxation of set $X$. 
Note that the constraints $\eta \geq g(\boldsymbol{Q}, \boldsymbol{u}, \boldsymbol{\psi}, \boldsymbol{x}, \boldsymbol{y}, \boldsymbol{z}), \forall(\boldsymbol{x}, \boldsymbol{y}, \boldsymbol{z}) \in \Xi_{C}$ is equivalent to

$$
\eta \geq \max _{(\boldsymbol{x}, \boldsymbol{y}, \boldsymbol{z}) \in \Xi_{C}}\{g(\boldsymbol{Q}, \boldsymbol{u}, \boldsymbol{\psi}, \boldsymbol{x}, \boldsymbol{y}, \boldsymbol{z})\},
$$

where the right-hand side is a linear program with nonempty and bounded feasible region for any given $(\boldsymbol{Q}, \boldsymbol{u}, \boldsymbol{\psi})$. Therefore, according to the strong duality of linear program, we can replace the max operator by its dual, i.e., an equivalent min operator, and further change the min operator with the existence one. Let $\varpi, \sigma, \rho, \zeta, \xi$ be the dual variables associated with constraints (22b), 22c , 22d,, $\boldsymbol{z} \leq \boldsymbol{e}$ and $\boldsymbol{x} \leq \boldsymbol{e}$, respectively. Then the conservative approximation (27) is equivalent to the following MILP:

$$
\begin{aligned}
& v^{C A}=\max _{\boldsymbol{Q}, \boldsymbol{u}, \boldsymbol{v}, \eta, \varpi, \boldsymbol{\sigma}, \boldsymbol{\rho}, \boldsymbol{\zeta}, \boldsymbol{\xi}} \sum_{i \in[n]} \bar{P}_{i} Q_{i}-\eta, \\
& \text { s.t. } \eta \geq k \varpi+\sum_{i \in[n]}\left(\zeta_{i}+\xi_{i}\right), \\
& \varpi+\zeta_{j}-\sum_{i \in[n]} \rho_{j i} \geq 0, \forall j \in[n], \\
& \sigma_{j i}+\rho_{j i} \geq-\bar{S}_{i} \underline{\alpha}_{j i}\left(u_{j}-\psi_{j}\right), \forall i, j \in[n], i \neq j, \\
& \sigma_{j j}+\rho_{j j} \geq \bar{S}_{j} l_{j}, \forall j \in[n], \\
& \xi_{i}-\sum_{j \in[n]} \sigma_{j i} \geq \bar{S}_{i}\left(Q_{i}-D_{i}\right)-\bar{S}_{i} \sum_{j \in[n]} \underline{\alpha}_{j i} \psi_{j}, \forall i \in[n], \\
& \zeta_{i}, \xi_{i}, \sigma_{i j}, \rho_{i j} \geq 0, \forall i, j \in[n], \\
& \text { (23a) - 23e }, 24 \mathrm{c}) \text {. }
\end{aligned}
$$

The following result summarizes the above development of the conservative approximation and also shows that under some sufficient conditions, this approximation can be exact, i.e., $v^{C A}=v^{*}$.

Theorem 5. Let $v^{C A}$ denote the optimal value of Model (28). Then

(i) $v^{C A} \leq v^{*}$; and

(ii) $v^{C A}=v^{*}$, if one of the following conditions holds: (1) $\underline{\boldsymbol{\alpha}}=\mathbf{0}$, or (2) $n=k$.

Proof. See Appendix A.6.

From Theorem 5, we see that the conservative approximation (28) provides a feasible solution to R-MNMS (24). In addition, Theorem 5 tells that the conservative approximation can provide a good-quality, even an optimal solution to R-MNMS (24). We will illustrate these facts in Section 6 . Please note that we provide closed-form solutions for the special cases when $\underline{\boldsymbol{\alpha}}=\mathbf{0}$ and $n=k$ in Section 4.2 and Section 4.3 , respectively. 


\section{Computational Study}

In this section, we test the performances of the branch and cut algorithm and conservative approximation to solve R-MNMS (24). Also, we study the impact of demand correlation on the total profit and test the reliability of R-MNMS 24) compared with its risk-neutral counterpart.

\subsection{Effectiveness of Algorithms}

We considered instances with $n=10$ and $n=20$ products. For each $n \in\{10,20\}$, we generated 10 random instances, where for each product $i \in[n]$, the nominal demand $D_{i}$ is between 50 and 100 , the unit price $p_{i}$ ranged from 85 to 95 , unit $\operatorname{cost} c_{i}$ varied from 40 to 50 , and the salvage value $s_{i}$ was between 22 and 30. All the products were assumed to be similar, we assume $k^{\alpha}=n^{2}-n$, and thus the substitution rates were generated uniformly between 0 and 1 , satisfying $\sum_{i \in[n]} \underline{\alpha}_{j i}=0.8$ and $\underline{\alpha}_{j j}=0$ for each $j \in[n]$. The lower bound of the demand was set to be proportional to the nominal demand, i.e., $\boldsymbol{l}=\theta \boldsymbol{D}$, where $\theta \in(0,1)$ is called "deviation ratio". We tested these instances with the budget of uncertainty $k \in\{2,5,8,10\}$ and the deviation ratio $\theta \in\{0.1,0.2,0.3,0.4,0.5\}$ for $n=10$, while for $n=20$, we tested the instances with $\theta \in\{0.2,0.4\}$ and $k \in\{5,10\}$. Both approaches were coded in Python 2.7 with calls to Gurobi 7.5 on a personal computer with 2.3 $\mathrm{GHz}$ Intel Core $\mathrm{i} 5$ processor and 8G of memory. The CPU time limit of Gurobi was set to be 3600 seconds.

Table 12 in Appendix C and Table 2 display the computational results of branch and cut algorithm and conservative approximation method with $n=10$ and $n=20$, respectively. Due to the length, we calculate the average computation result of Table 12 for each parametric setting in Table 1. For the branch and cut algorithm, Opt.val denotes the optimal value if available, LB and UB denote best lower and upper bounds, Gap denotes the optimality gap, computed as (UB-LB)/UB; for conservative approximation, C.val denotes its output objective value, and A-Gap represents its optimality gap, computed as (UB-C.val)/UB (note that UB is equal to the Opt.val if available).

From Table 1 or Table 12 in Appendix C, we see that when $n=10$, both approaches find goodquality feasible solutions. As explained in Section 5.3, the solutions obtained from conservative approximation method are equal to the solutions calculated from branch and cut algorithm when $k=n=10$, i.e., A-Gap=0. In Table 2, we note that when the number of products increases to 20, the conservative approximation method can find good-quality feasible solutions within the time limit. Oftentimes, the conservative approximation solution can be even better than that obtained by the branch and cut algorithm. Also, from Table 2, we see that if the budget of uncertainty $k$ increases and $k \leq \frac{n}{2}$, i.e., the number of possible realizations of products' demand grows, then the computational time tends to be longer. Also, since a larger $k$ implies that a larger number of products whose worst-case demand can be equal to their lower bounds, we can anticipate that the total profit becomes smaller. Since $\theta$ denotes how much the worst-case demand can deviate from 
the nominal demand, the increase of $\theta$ implies that the variance of random demand grows, which means a chance of being understock or overstock becomes larger and leads to a smaller total profit.

Table 1: Average computational results of branch and cut algorithm, and conservative approximation algorithm with $n=10$.

\begin{tabular}{|c|c|c|c|c|c|c|}
\hline \multirow{2}{*}{$k$} & \multirow{2}{*}{$\theta$} & \multicolumn{2}{|c|}{ Branch and Cut } & \multicolumn{3}{|c|}{ Conservative Approximation } \\
\hline & & Average Time & Average Opt.val & Average Time & Average C.val & Average A-Gap(\%) \\
\hline 2 & \multirow{4}{*}{0.1} & 5.14 & 35117.2 & 4.66 & 35111.4 & 0.02 \\
\hline 5 & & 6.71 & 33647.7 & 8.21 & 33587.5 & 0.18 \\
\hline 8 & & 13.82 & 32914 & 22.26 & 32710.6 & 0.62 \\
\hline 10 & & 1.04 & 32516.4 & 4.45 & 32708.5 & 0 \\
\hline 2 & \multirow{4}{*}{0.2} & 4.73 & 33684.1 & 4.9 & 33880.5 & 0.04 \\
\hline 5 & & 6.43 & 30952.6 & 9.72 & 30833.6 & 0.38 \\
\hline 8 & & 14.35 & 29485.1 & 16.37 & 29078.4 & 1.38 \\
\hline 10 & & 0.95 & 29074.2 & 4.18 & 29074.2 & 0 \\
\hline 2 & \multirow{4}{*}{0.3} & 4.48 & 32665.6 & 4.92 & 32649.1 & 0.05 \\
\hline 5 & & 6.85 & 28257.4 & 9.28 & 28079.2 & 0.63 \\
\hline 8 & & 17.7 & 26056.3 & 13.78 & 25296.7 & 2.34 \\
\hline 10 & & 1.11 & 25440 & 3.27 & 25440 & 0 \\
\hline 2 & \multirow{4}{*}{0.4} & 5.13 & 31439.8 & 4.9 & 31214.9 & 0.07 \\
\hline 5 & & 6.61 & 25562.3 & 8.27 & 25324.6 & 0.94 \\
\hline 8 & & 15.47 & 22627.4 & 11.68 & 21814.1 & 3.59 \\
\hline 10 & & 1.02 & 21805.7 & 3.18 & 21677.6 & 0 \\
\hline 2 & \multirow{4}{*}{0.5} & 5.51 & 30213.8 & 5.16 & 30187.4 & 0.09 \\
\hline 5 & & 7.7 & 22867.1 & 7.95 & 22570.9 & 1.3 \\
\hline 8 & & 17.46 & 19198.6 & 10.37 & 18181.9 & 5.29 \\
\hline 10 & & 1.58 & 18171.4 & 1.78 & 18171.4 & 0 \\
\hline
\end{tabular}

\subsection{Robustness of Model (24)}

In this subsection, we illustrate how to find the optimal budget of uncertainty and also test robustness of Model (24). Suppose that there are 10 products. The values of $\boldsymbol{p}, \boldsymbol{c}, \boldsymbol{s}, \underline{\boldsymbol{\alpha}}$, and $k^{\alpha}$ are the same as those in Section 6.1. We also assumed that there are 200 historical demand data points available and we split them into two groups, $\Upsilon_{1}, \Upsilon_{2}$, with equal size. These historical demand data points were generated by sampling from independent uniformly random variables between 20 and 80. We choose the candidate set $\mathcal{K}$ of budget of uncertainty $k$ as $\{0,1, \cdots, 10\}$. According to Section 2.2 with percentile $q=10$, we found the optimal budget of uncertainty $k^{*}=6$, which is the smallest $k \in \mathcal{K}$ such that $v^{*}\left(k, k^{\alpha}\right) \leq \widehat{\Pi}^{10 \%}\left(k, k^{\alpha}\right)$ as shown in Figure 1. In Table 13 of Appendix D, we further illustrate how to follow the similar procedure to jointly choose the best pair of $\left(k, k^{\alpha}\right)$. 
Table 2: Computational results of branch and cut algorithm and conservative approximation method with $n=20$.

\begin{tabular}{|c|c|c|c|c|c|c|c|c|c|}
\hline \multirow{2}{*}{$k$} & \multirow{2}{*}{$\theta$} & \multirow{2}{*}{ Instances } & \multicolumn{4}{|c|}{ Branch and Cut } & \multicolumn{3}{|c|}{ Conservative Approximation } \\
\hline & & & Time & LB & UB & Gap (\%) & Time & C.val & A-Gap (\%) \\
\hline \multirow{10}{*}{5} & \multirow{10}{*}{0.2} & 1 & 3600 & 62696.6 & 64708.6 & 3.21 & 426 & 63598.0 & 1.72 \\
\hline & & 2 & 3600 & 57808.9 & 59814.1 & 3.47 & 376 & 58676.9 & 1.90 \\
\hline & & 3 & 3600 & 67405.0 & 69220.8 & 2.69 & 386 & 68271.3 & 1.37 \\
\hline & & 4 & 3600 & 63595.0 & 65665.0 & 3.26 & 474 & 64289.4 & 2.10 \\
\hline & & 5 & 3600 & 68212.6 & 70193.6 & 2.90 & 483 & 68970.1 & 1.74 \\
\hline & & 6 & 3600 & 63162.1 & 65226.0 & 3.27 & 389 & 64158.1 & 1.64 \\
\hline & & 7 & 3600 & 57161.3 & 58977.5 & 3.18 & 443 & 57873.8 & 1.87 \\
\hline & & 8 & 3600 & 59865.9 & 61533.3 & 2.79 & 399 & 60482.0 & 1.71 \\
\hline & & 9 & 3600 & 63039.5 & 64734.2 & 2.69 & 297 & 64015.2 & 1.11 \\
\hline & & 10 & 3600 & 63736.1 & 65823.4 & 3.28 & 445 & 64440.0 & 2.10 \\
\hline \multicolumn{3}{|c|}{ Average } & 3600 & 62668.3 & 64589.6 & 2.98 & 412 & 63477.5 & 1.73 \\
\hline \multirow{10}{*}{5} & \multirow{10}{*}{0.4} & 1 & 3600 & 56885.9 & 59198.5 & 4.07 & 209 & 57605.3 & 2.69 \\
\hline & & 2 & 3600 & 52545.4 & 55277.5 & 5.20 & 164 & 53302.7 & 3.57 \\
\hline & & 3 & 3600 & 61266.0 & 63481.5 & 3.62 & 210 & 62016.9 & 2.31 \\
\hline & & 4 & 3600 & 57894.0 & 60005.2 & 3.65 & 171 & 58491.9 & 2.52 \\
\hline & & 5 & 3600 & 62189.9 & 64349.5 & 3.47 & 214 & 62787.7 & 2.43 \\
\hline & & 6 & 3600 & 57600.8 & 59577.6 & 3.43 & 162 & 58411.6 & 1.96 \\
\hline & & 7 & 3600 & 51958.4 & 53876.0 & 3.69 & 188 & 52501.2 & 2.55 \\
\hline & & 8 & 3600 & 54290.0 & 56740.3 & 4.51 & 166 & 54681.1 & 3.63 \\
\hline & & 9 & 3600 & 57439.3 & 60157.6 & 4.73 & 162 & 58052.8 & 3.50 \\
\hline & & 10 & 3600 & 57968.5 & 60021.7 & 3.54 & 193 & 58443.9 & 2.63 \\
\hline \multicolumn{3}{|c|}{ Average } & 3600 & 57003.8 & 59268.5 & 4.54 & 184 & 57629.5 & 3.96 \\
\hline \multirow{10}{*}{10} & \multirow{10}{*}{0.2} & 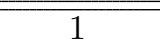 & 3600 & 58550.7 & 61391.8 & $\overline{74.85}$ & 2677 & 58760.5 & 4.29 \\
\hline & & 2 & 3600 & 53961.4 & 55228.0 & 2.35 & 676 & 54308.9 & 1.66 \\
\hline & & 3 & 3600 & 62883.8 & 66951.2 & 6.47 & 674 & 62988.8 & 5.92 \\
\hline & & 4 & 3600 & 59129.6 & 62307.3 & 5.37 & 744 & 59423.0 & 4.63 \\
\hline & & 5 & 3600 & 63215.9 & 65995.8 & 5.37 & 775 & 63774.9 & 3.37 \\
\hline & & 6 & 3600 & 58661.4 & 61816.1 & 5.38 & 588 & 59310.5 & 4.05 \\
\hline & & 7 & 3600 & 53268.3 & 56034.5 & 4.40 & 679 & 53631.6 & 4.29 \\
\hline & & 8 & 3600 & 55993.3 & 58219.7 & 3.98 & 584 & 56143.3 & 3.57 \\
\hline & & 9 & 3600 & 58682.7 & 61631.3 & 5.03 & 519 & 59298.9 & 3.79 \\
\hline & & 10 & 3600 & 59474.8 & 62217.1 & 4.61 & 667 & 59677.8 & 4.08 \\
\hline \multicolumn{3}{|c|}{ Average } & 3600 & 58382.2 & 61179.3 & 3.84 & 658 & 58731.8 & 2.78 \\
\hline \multirow{11}{*}{10} & \multirow{10}{*}{0.4} & 1 & 3600 & 48742.5 & 50596.0 & 3.80 & 192 & 48090.8 & 4.95 \\
\hline & & 2 & 3600 & 44928.1 & 46423.0 & 3.33 & 132 & 44626.2 & 3.87 \\
\hline & & 3 & 3600 & 52462.0 & 54713.1 & 4.29 & 162 & 51612.7 & 5.67 \\
\hline & & 4 & 3600 & 49276.9 & 51014.4 & 3.53 & 149 & 48758.0 & 4.42 \\
\hline & & 5 & 3600 & 52576.1 & 55947.0 & 6.41 & 137 & 52511.7 & 6.14 \\
\hline & & 6 & 3600 & 48995.8 & 50832.1 & 3.75 & 113 & 48792.0 & 4.01 \\
\hline & & 7 & 3600 & 44435.2 & 45413.2 & 2.20 & 133 & 44066.6 & 2.97 \\
\hline & & 8 & 3600 & 46552.2 & 47417.1 & 1.86 & 141 & 45977.6 & 3.04 \\
\hline & & 9 & 3600 & 49148.4 & 50407.4 & 2.56 & 117 & 48861.3 & 3.07 \\
\hline & & 10 & 3600 & 49663.0 & 50784.3 & 2.26 & 183 & 48956.5 & 3.60 \\
\hline & \multicolumn{2}{|c|}{ Average } & 3600 & 48678.0 & 50354.8 & 3.27 & 146 & 48225.3 & 4.20 \\
\hline
\end{tabular}




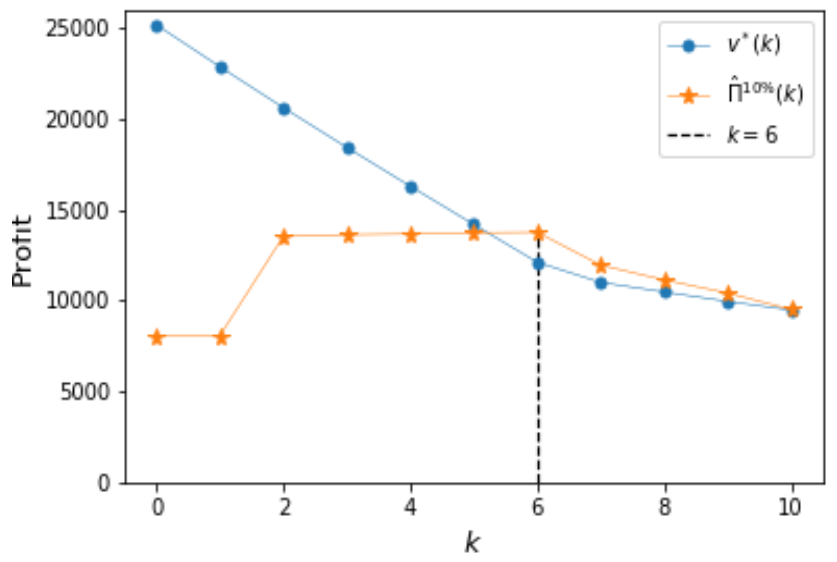

Figure 1: The 10th percentile of profits for Model (2) by plugging in the optimal order quantities of robust model 24 for different $k$.

We also tested the reliability of the solution from robust Model (24) by comparing with the risk neutral one studied in Zhang et al. (2018), which has the following form:

$$
v^{r n}=\max _{\boldsymbol{Q} \in \mathbb{R}_{+}^{n}}\left\{\sum_{i \in[n]} \bar{P}_{i} Q_{i}-\mathbb{E}_{\mathbb{P}}\left[\sum_{i \in[n]} \bar{S}_{i}\left(Q_{i}-\tilde{D}_{i}^{s}(\boldsymbol{Q})\right)_{+}\right]\right\},
$$

where $\mathbb{P}$ denotes a particular probability distribution.

We first used the demand data in $\Upsilon_{2}$ to obtain the optimal order quantities of robust Model (24) with $k^{*}=6$. Also, we obtained the optimal order quantities from the risk-neutral Model (29) by solving the sampling average approximation (SAA) with the demand realizations from set $\Upsilon_{2}$. To compare the quality of both solutions, we assumed that the underlying true probability distribution of each product's demand is independent Gaussian $\mathcal{N}\left(\mu, \sigma^{2}\right)$ truncated at the interval $[20,80]$. We selected different parametric pairs $\left(\mu, \sigma^{2}\right)$ of Gaussian random vectors, and for each pair $\left(\mu, \sigma^{2}\right)$, we generated $10^{5}$ i.i.d. samples to evaluate the solutions from robust Model (24) and risk-neutral Model (29) and also to compute their statistical confidence intervals. The computational results are presented in Table 3 .

From Table 3, we see that if the data are very limited and unable to predict the underlying true probability distribution or if the underlying true probability distribution is not the same as the one we stick to, then the solution from robust Model (24) is more reliable than that from risk-neutral Model (29). On the other hand, if the underlying true distribution is close to the one we predict using the historical data (e.g., in the cases of Gaussian $\left(40,40^{2}\right)$ and Gaussian $\left(40,50^{2}\right)$ ), then riskneutral Model $(29)$ can be more accurate. In practice, if there are limited data or the demand is changing rapidly, then we recommend the robust Model (24), and if there are plenty of historical 
data and products' demand is quite stable, then risk-neutral Model 290 is more desirable.

Table 3: Mean and 95\% Confidence Interval (CI) objective value of robust Model 24) and risk neutral Model 29. under different Gaussian distributions.

\begin{tabular}{c|c|c}
\hline Distribution & Mean and 95\% CI of Robust Model & Mean and 95\% CI of Risk Neutral Model \\
\hline Gaussian $\left(20,10^{2}\right)$ & $6416.22 \pm 6.29$ & $5066.66 \pm 6.33$ \\
Gaussian $\left(20,40^{2}\right)$ & $11559.16 \pm 15.64$ & $10951.76 \pm 17.74$ \\
Gaussian $\left(20,50^{2}\right)$ & $12316.00 \pm 16.38$ & $11929.89 \pm 18.96$ \\
Gaussian $\left(30,10^{2}\right)$ & $10875.82 \pm 9.24$ & $9554.59 \pm 9.30$ \\
Gaussian $\left(30,50^{2}\right)$ & $13643.80 \pm 16.16$ & $13387.78 \pm 18.70$ \\
Gaussian $\left(30,80^{2}\right)$ & $14013.59 \pm 16.63$ & $13959.36 \pm 19.53$ \\
Gaussian $\left(40,10^{2}\right)$ & $16618.50 \pm 9.61$ & $15553.12 \pm 10.27$ \\
Gaussian $\left(40,40^{2}\right)$ & $15780.94 \pm 15.98$ & $15964.15 \pm 18.90$ \\
Gaussian $\left(40,50^{2}\right)$ & $15734.04 \pm 16.32$ & $16037.57 \pm 19.47$ \\
\hline
\end{tabular}

\subsection{Impact of Demand Correlation}

In this subsection, we analyze the impact of the correlation coefficient of the demand on the total profit for different levels of budget of uncertainty $k$. Suppose that there are 10 products. The values of $\boldsymbol{p}, \boldsymbol{c}, \boldsymbol{s}, \underline{\boldsymbol{\alpha}}$, and $k^{\alpha}$ are the same as those in Section 6.1. Given i.i.d. uniform random variables $\left\{\tilde{U}_{i}\right\}_{i \in\{0\} \cup[n]}$ distributed between -1 and 1 , we assume that the random demand of $i$ th product is $\tilde{D}_{i}=50+30\left(\hat{\rho} \tilde{U}_{0}+(1-\widehat{\rho}) \tilde{U}_{i}\right)$ for each $i \in[n]$. Theoretically, the Pearson correlation coefficient between any two distinct products $i$ and $j$ is given by $\widehat{\rho}^{2} /\left(\widehat{\rho}^{2}+(1-\widehat{\rho})^{2}\right)$ (please find the derivation in Appendix E), increasing with the increase in $\widehat{\rho}$ for $\widehat{\rho} \in[0,1]$. We constructed the demand uncertainty set $\mathcal{U}$ as follows: (i) We first generated 100 data points from the probability distribution of $\tilde{U}_{i}$ for each $i \in\{0\} \cup[n]$, denoted by $\left\{U_{0, l}\right\}_{l \in[100]},\left\{U_{1, l}\right\}_{l \in[100]}, \cdots,\left\{U_{10, l}\right\}_{l \in[100]}$; (ii) Next, we calculated 100 demand points $\left\{D_{i, l}\right\}_{l \in[100]}$ using the formula $D_{i, l}=50+30\left(\widehat{\rho} U_{0, l}+(1-\widehat{\rho}) U_{i, l}\right)$ for all $i \in[n]$ and $l \in[100]$; and (iii) Finally, we used these 100 demand points of $\left\{D_{i, l}\right\}_{i \in[n], l \in[100]}$ to construct the uncertainty set $\mathcal{U}$ following from the steps in Section 2.2. The optimal profits of the robust Model (24) for different budgets of uncertainty $k$ are illustrated in Figure 2, It is seen that when $k$ is small, the profit decreases when $\widehat{\rho}$ increases (i.e., the demand correlation coefficient increases). This trend diminishes as $k$ is close to $n$, which is mainly because the impact of the correlation on the profit is dominated by that of $k$. Besides, as $k$ increases, the worst-case demand of most of products is equal to its lower bound according to robust model (24), which can be over-conservative and significantly reduces the total profit.

\section{Conclusion}

This work studies the robust multi-product newsvendor problem with substitution (R-MNMS), where the demand and substitution are under cardinality-constrained uncertainty sets. We first prove that evaluating the worst-case total profit for given order quantities, in general, is NP-hard. Next, we identify three solvable special cases of R-MNMS and derive their closed-form optimal 


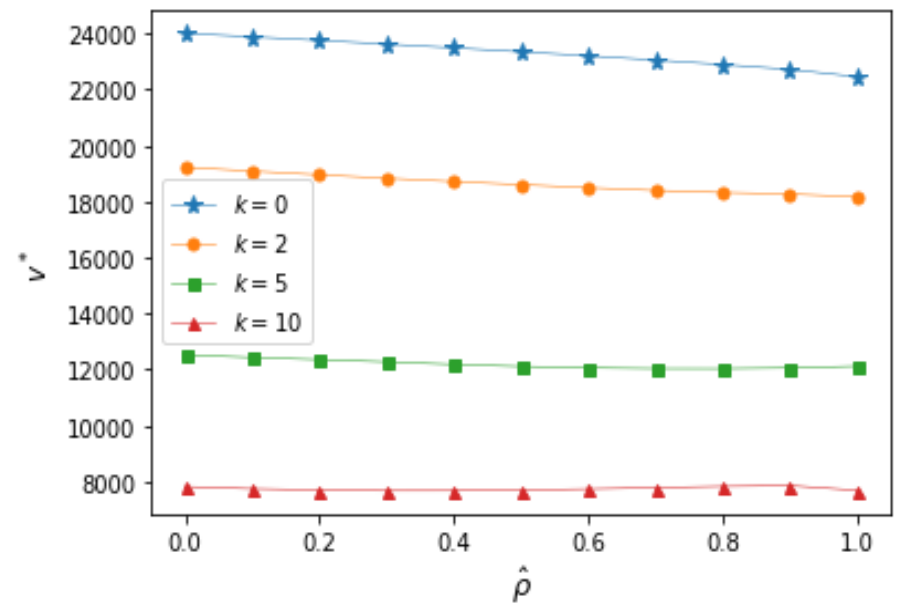

Figure 2: Impact of correlation on the profit of robust model 24 at different budgets of uncertainty $k$.

solutions. For a general R-MNMS, we propose a mixed-integer linear program formulation that can be solved by a branch and cut algorithm. We also develop a conservative approximation method to solve R-MNMS and show that under certain conditions, its optimal solution can also be optimal to R-MNMS. Finally, we conduct numerical studies to illustrate the effectiveness and solution quality of the proposed algorithms. Please note that the cardinality uncertainty set is essential for the results in this paper, which might not hold if one changes to other uncertainty sets. One possible future direction is to extend the robust model to other interesting data-driven uncertainty sets, for example, moment-based uncertainty sets (Scarf, 1957; Xie and Ahmed, 2018b; $\mathrm{Li}$ and $\mathrm{Fu}, 2017)$. Another direction is to incorporate pricing decisions into R-MNMS, i.e., to study joint inventory and pricing optimization in R-MNMS. Finally, to explore the correlation between the stochastic substitution rates and stochastic demand, i.e., to study R-MNMS under a joint uncertainty in demand and the substitution rates is also interesting.

\section{Acknowledgments}

The authors thank the referees and editors for the helpful comments.

\section{References}

Addis, B., Carello, G., Grosso, A., Lanzarone, E., Mattia, S., Tànfani, E., 2015. Handling uncertainty in health care management using the cardinality-constrained approach: Advantages and remarks. Operations Research for Health Care 4, 1-4.

Anupindi, R., Dada, M., Gupta, S., 1998. Estimation of consumer demand with stock-out based substitution: An application to vending machine products. Marketing Science 17, 406-423.

Baker, R., Urban, T.L., 1988. Single-period inventory dependent demand models. Omega 16, 605-607. 
Balakrishnan, A., Pangburn, M.S., Stavrulaki, E., 2004. stack them high, letem fly: Lot-sizing policies when inventories stimulate demand. Management Science 50, 630-644.

Balakrishnan, A., Pangburn, M.S., Stavrulaki, E., 2008. Integrating the promotional and service roles of retail inventories. Manufacturing \& Service Operations Management 10, 218-235.

Bassok, Y., Anupindi, R., Akella, R., 1999. Single-period multiproduct inventory models with substitution. Operations Research 47, 632-642.

Bertsimas, D., Sim, M., 2004. The price of robustness. Operations research 52, 35-53.

Bertsimas, D., Thiele, A., 2006. A robust optimization approach to inventory theory. Operations research 54, $150-168$.

Bienstock, D., ÖZbay, N., 2008. Computing robust basestock levels. Discrete Optimization 5, 389-414.

Carello, G., Lanzarone, E., 2014. A cardinality-constrained robust model for the assignment problem in home care services. European Journal of Operational Research 236, 748-762.

Choi, T.M., 2012. Handbook of Newsvendor problems: Models, extensions and applications. volume 176. Springer.

Chopra, S., Meindl, P., 2007. Supply chain management. strategy, planning \& operation. Das summa summarum des management, 265-275.

Dantzig, G.B., 1957. Discrete-variable extremum problems. Operations research 5, 266-288.

Eliashberg, J., Steinberg, R., 1993. Marketing-production joint decision-making. Handbooks in operations research and management science 5, 827-880.

Erlebacher, S.J., 2000. Optimal and heuristic solutions for the multi-item newsvendor problem with a single capacity constraint. Production and Operations Management 9, 303-318.

Gerchak, Y., Wang, Y., 1994. Periodic-review inventory models with inventory-level-dependent demand. Naval Research Logistics (NRL) 41, 99-116.

HazıR, Ö., Dolgui, A., 2013. Assembly line balancing under uncertainty: Robust optimization models and exact solution method. Computers \& Industrial Engineering 65, 261-267.

Huang, D., Zhou, H., Zhao, Q.H., 2011. A competitive multiple-product newsboy problem with partial product substitution. Omega 39, 302-312.

Jiang, H., Netessine, S., Savin, S., 2011. Robust newsvendor competition under asymmetric information. Operations research 59, 254-261.

Kök, A.G., Fisher, M.L., 2007. Demand estimation and assortment optimization under substitution: Methodology and application. Operations Research 55, 1001-1021.

Lanzarone, E., Matta, A., 2012. The nurse-to-patient assignment problem in home care services, in: Advanced Decision Making Methods Applied to Health Care. Springer, pp. 121-139.

Levi, R., Perakis, G., Romero, G., 2014. A continuous knapsack problem with separable convex utilities: Approximation algorithms and applications. Operations Research Letters 42, 367-373.

Li, Z., Fu, Q.G., 2017. Robust inventory management with stock-out substitution. International Journal of Production Economics 193, 813-826.

Loedy, N., Lesmono, D., Limansyah, T., 2018. An inventory-dependent demand model with deterioration, all-units discount, and return, in: IOP Conf. Series: Journal of Physics, p. 012010.

Lu, C.C., Ying, K.C., Lin, S.W., 2014. Robust single machine scheduling for minimizing total flow time in the presence of uncertain processing times. Computers \& Industrial Engineering 74, 102-110.

Lugaresi, G., 2016. The cardinality-constrained approach applied to manufacturing problems .

Lugaresi, G., Lanzarone, E., Frigerio, N., Matta, A., 2017. A cardinality-constrained approach for robust machine loading problems. Procedia Manufacturing 11, 1718-1725.

Maity, K., Maiti, M., 2005. Inventory of deteriorating complementary and substitute items with stock dependent demand. American Journal of Mathematical and Management Sciences 25, 83-96.

McCormick, G.P., 1976. Computability of global solutions to factorable nonconvex programs: Part i?convex under- 
estimating problems. Mathematical programming 10, 147-175.

Moon, Y., Yao, T., 2011. A robust mean absolute deviation model for portfolio optimization. Computers \& Operations Research 38, 1251-1258.

Moreira, M.C.O., Cordeau, J.F., Costa, A.M., Laporte, G., 2015. Robust assembly line balancing with heterogeneous workers. Computers \& Industrial Engineering 88, 254-263.

Müller, S., Huber, J., Fleischmann, M., Stuckenschmidt, H., 2020. Data-driven inventory management under customer substitution. Available at SSRN 3624026 .

Nahmias, S., Olsen, T.L., 2015. Production and operations analysis. Waveland Press.

Netessine, S., Rudi, N., 2003. Centralized and competitive inventory models with demand substitution. Operations research 51, 329-335.

Padberg, M., Rinaldi, G., 1991. A branch-and-cut algorithm for the resolution of large-scale symmetric traveling salesman problems. SIAM review 33, 60-100.

Rajaram, K., Tang, C.S., 2001. The impact of product substitution on retail merchandising. European Journal of Operational Research 135, 582-601.

Rao, U.S., Swaminathan, J.M., Zhang, J., 2004. Multi-product inventory planning with downward substitution, stochastic demand and setup costs. IIE Transactions 36, 59-71.

Scarf, H.E., 1957. A min-max solution of an inventory problem. Technical Report. RAND CORP SANTA MONICA CALIF.

Schrijver, A., 1998. Theory of linear and integer programming. John Wiley \& Sons.

Schweitzer, M.E., Cachon, G.P., 2000. Decision bias in the newsvendor problem with a known demand distribution: Experimental evidence. Management Science 46, 404-420.

Sen, S., Sherali, H.D., 2006. Decomposition with branch-and-cut approaches for two-stage stochastic mixed-integer programming. Mathematical Programming 106, 203-223.

Shin, H., Park, S., Lee, E., Benton, W., 2015. A classification of the literature on the planning of substitutable products. European Journal of Operational Research 246, 686-699.

Shumsky, R.A., Zhang, F., 2009. Dynamic capacity management with substitution. Operations research 57, 671-684.

Sion, M., 1958. On general minimax theorems. Pacific Journal of mathematics 8, 171-176.

Solyal,, O., Cordeau, J.F., Laporte, G., 2012. Robust inventory routing under demand uncertainty. Transportation Science 46, 327-340.

Stavrulaki, E., 2011. Inventory decisions for substitutable products with stock-dependent demand. International Journal of Production Economics 129, 65-78.

Vaagen, H., Wallace, S.W., Kaut, M., 2011. Modelling consumer-directed substitution. International Journal of Production Economics 134, 388-397.

Xie, W., Ahmed, S., 2018a. Distributionally robust chance constrained optimal power flow with renewables: A conic reformulation. IEEE Transactions on Power Systems 33, 1860-1867.

Xie, W., Ahmed, S., 2018b. On deterministic reformulations of distributionally robust joint chance constrained optimization problems. SIAM Journal on Optimization 28, 1151-1182.

Yu, Y., Chen, X., Zhang, F., 2015. Dynamic capacity management with general upgrading. Operations Research 63, 1372-1389.

Zhang, J., Xie, W., Sarin, S., 2018. Multi-product newsvendor problem with customer-driven demand substitution: A stochastic integer program perspective. 


\section{Appendix A. Proofs}

\section{A.1 Proof of Proposition 2}

Proposition 2. There exists an optimal solution $\boldsymbol{Q}^{*}$ to $R$-MNMS such that for each product $i \in[n]$, $Q_{i}^{*} \leq M_{i}$, where $M_{i}=D_{i}+\sum_{j \in[n]} \underline{\alpha}_{j i} D_{j}$.

Proof. We prove the result by contradiction. Suppose for any optimal solution $\boldsymbol{Q}^{*}$, there exists a product $i \in[n]$ such that $Q_{i}^{*}>M_{i}$. Let set $\mathbb{B}:=\left\{i \in[n]: Q_{i}^{*}>M_{i}\right\}$. Hence, $\mathbb{B} \neq \emptyset$. Let us define a new solution $\widehat{\boldsymbol{Q}}$ such that $\widehat{Q}_{i}=\left\{\begin{array}{ll}M_{i}, & \text { if } i \in \mathbb{B} \\ Q_{i}^{*}, & \text { otherwise }\end{array}\right.$ for each product $i \in[n]$. Clearly, $\widehat{Q}_{i} \leq M_{i}$ for each $i \in[n]$. Then the objective value $f(\widehat{\boldsymbol{Q}})$ is equal to

$$
\begin{aligned}
f(\widehat{\boldsymbol{Q}})= & \sum_{i \in \mathbb{B}} \bar{P}_{i} M_{i}+\sum_{i \in[n] \backslash \mathbb{B}} \bar{P}_{i} Q_{i}^{*} \\
& -\max _{\boldsymbol{z} \in X}\left\{\sum_{i \in \mathbb{B}} \bar{S}_{i}\left(M_{i}-D_{i}+l_{i} z_{i}-\sum_{j \in \mathbb{B}} \underline{\alpha}_{j i}\left(D_{j}-l_{j} z_{j}-M_{j}\right)_{+}-\sum_{j \in[n] \backslash \mathbb{B}} \underline{\alpha}_{j i}\left(D_{j}-l_{j} z_{j}-Q_{j}^{*}\right)_{+}\right)_{+}\right. \\
& \left.+\sum_{i \in[n] \backslash \mathbb{B}} \bar{S}_{i}\left(Q_{i}^{*}-D_{i}+l_{i} z_{i}-\sum_{j \in \mathbb{B}} \underline{\alpha}_{j i}\left(D_{j}-l_{j} z_{j}-M_{j}\right)_{+}-\sum_{j \in[n] \backslash \mathbb{B}} \underline{\alpha}_{j i}\left(D_{j}-l_{j} z_{j}-Q_{j}^{*}\right)_{+}\right)_{+}\right\} \\
= & \sum_{i \in \mathbb{B}} \bar{P}_{i} M_{i}+\sum_{i \in[n] \backslash \mathbb{B}} \bar{P}_{i} Q_{i}^{*}+\sum_{i \in \mathbb{B}} \bar{S}_{i}\left(Q_{i}^{*}-M_{i}\right) \\
& -\max _{z \in X}\left\{\sum_{i \in \mathbb{B}} \bar{S}_{i}\left(Q_{i}^{*}-D_{i}+l_{i} z_{i}-\sum_{j \in[n] \backslash \mathbb{B}} \underline{\alpha}_{j i}\left(D_{j}-l_{j} z_{j}-Q_{j}^{*}\right)_{+}\right)\right. \\
& \left.+\sum_{i \in[n] \backslash \mathbb{B}} \bar{S}_{i}\left(Q_{i}^{*}-D_{i}+l_{i} z_{i}-\sum_{j \in[n] \backslash \mathbb{B}} \underline{\alpha}_{j i}\left(D_{j}-l_{j} z_{j}-Q_{j}^{*}\right)_{+}\right)_{+}\right\} \\
= & \sum_{i \in[n]} \bar{P}_{i} Q_{i}^{*}-\max _{\boldsymbol{z} \in X}\left\{\sum_{i \in[n]} \bar{S}_{i}\left(Q_{i}^{*}-D_{i}+l_{i} z_{i}-\sum_{j \in[n]} \underline{\alpha}_{j i}\left(D_{j}-l_{j} z_{j}-Q_{j}^{*}\right)_{+}\right)_{+}\right\} \\
& +\sum_{i \in \mathbb{B}}\left(\bar{S}_{i}-\bar{P}_{i}\right)\left(Q_{i}^{*}-M_{i}\right) \\
= & v^{*}+\sum_{i \in \mathbb{B}}\left(\bar{S}_{i}-\bar{P}_{i}\right)\left(Q_{i}^{*}-M_{i}\right) \\
\geq v^{*}, &
\end{aligned}
$$

where the second equality is because of $M_{j}=D_{j}+\sum_{i \in[n]} \underline{\alpha}_{j i} D_{i}$ for all $j \in \mathbb{B}$, the third equality is because for each $j \in \mathbb{B}$, we have $Q_{j}^{*}>M_{j}=D_{j}+\sum_{i \in[n]} \underline{\alpha}_{i j} D_{i}$, the forth equality is due to the optimality of $Q^{*}$, and the last inequality holds because $\bar{S}_{i} \geq \bar{P}_{i}$ and $Q_{i}^{*}>M_{i}$ for each $i \in[n]$. This implies $\widehat{\boldsymbol{Q}}$ is also an optimal solution, a contradiction. 


\section{A.2 Proof of Theorem 1}

Theorem 1. The inner maximization problem 13] in general is NP-hard.

Proof. We prove this result from a reduction of clique problem to be a special case of Model (13).

(Clique Problem) Given an undirected graph $G(V, E)$, does it have a size- $\tau$ clique?

Let us consider a special instance of the inner maximization problem $(13)$ : suppose that there are $n=|V|+|E|$ products, and for each product $i \in E$, we let $\bar{S}_{i}=1, Q_{i}=D_{i}, l_{i}=1$, while for each product $j \in V$, we let $\bar{S}_{j}=1, Q_{j}=D_{j}-l_{j}, l_{j}=1$. Additionally, the substitution rate matrix $\underline{\boldsymbol{\alpha}}$ is defined as

$$
\underline{\alpha}_{j i}= \begin{cases}1, & \text { if edge } i \in E \text { contains node } j \in V \\ 0, & \text { otherwise }\end{cases}
$$

for all $i, j \in V \cup E$. Let the budget of uncertainty $k=\frac{\tau(\tau+1)}{2}$. Under this setting, the inner maximization problem 13 reduces to

$$
\begin{aligned}
R(\boldsymbol{Q})=\max _{\boldsymbol{z}} & \sum_{i \in E}\left(z_{i}^{(E)}-\sum_{j \in V} \underline{\alpha}_{j i}\left(1-z_{j}^{(V)}\right)\right)_{+}+\sum_{j \in V} z_{j}^{(V)}, \\
\text { s.t. } & \sum_{j \in V} z_{j}^{(V)}+\sum_{i \in E} z_{i}^{(E)} \leq \frac{\tau(\tau+1)}{2}, \\
& z_{j}^{(V)}, z_{i}^{(E)} \in\{0,1\} .
\end{aligned}
$$

It is sufficient to show that the Clique Problem is equivalent to Model (30), i.e., we only need to show the following claim.

Claim 1. There is a clique with $\tau$ nodes in the undirected graph $G(V, E)$ if and only if $R(\boldsymbol{Q})=$ $\frac{\tau(\tau+1)}{2}$.

Proof. Before we prove the result, let us denote $\boldsymbol{z}^{*}$ as an optimal solution of Model (30), and also define the following two sets: $V^{*}=\left\{j \in V:\left(z_{j}^{(V)}\right)^{*}=1\right\}, E^{*}=\left\{i \in E:\left(z_{i}^{(E)}\right)^{*}=1\right\}$, i.e., $\widehat{G}\left(V^{*}, E^{*}\right)$ is a substructure of $G(V, E)$. Note that $\widehat{G}\left(V^{*}, E^{*}\right)$ might not be a graph since we might not choose enough nodes to cover all the edges, i.e., there might exist an edge in set $E^{*}$ but not both of its two nodes are selected in set $V^{*}$. Thus, $R(\boldsymbol{Q})$ is equal to

$$
R(\boldsymbol{Q})=\sum_{i \in E^{*}}\left(1-\sum_{j \in V} \underline{\alpha}_{j i}\left(1-\left(z_{j}^{(V)}\right)^{*}\right)\right)_{+}+\sum_{j \in V^{*}}\left(z_{j}^{(V)}\right)^{*}=\sum_{i \in E^{*}}\left(1-\sum_{j \in V \backslash V^{*}} \underline{\alpha}_{j i}\right)_{+}+\left|V^{*}\right| .
$$


From (31), we have the following inequality:

$$
R(\boldsymbol{Q})=\sum_{i \in E^{*}}\left(1-\sum_{j \in V \backslash V^{*}} \underline{\alpha}_{j i}\right)_{+}+\left|V^{*}\right| \leq \sum_{i \in E}\left(1-\sum_{j \in V \backslash V^{*}} \underline{\alpha}_{j i}\right)_{+}+\left|V^{*}\right| \leq\left(\begin{array}{c}
\left|V^{*}\right| \\
2
\end{array}\right)+\left|V^{*}\right|,
$$

where the first inequality is due to $E^{*} \subseteq E$, and the second inequality is because of $\left(1-\sum_{j \in V \backslash V^{*}} \underline{\alpha}_{j i}\right)_{+}=$ 0 if at least one of the two nodes from edge $i$ is not covered by set $V^{*}$.

Now we are ready to prove the main results.

"only if". Suppose that there exists a size- $\tau$ clique $\left(V_{\tau}, E_{\tau}\right)$ in the graph $G(V, E)$. Let us denote a binary vector $\widehat{z}$ as

$$
\widehat{z}_{j}^{(V)}=\left\{\begin{array}{lc}
1, & j \in V_{\tau} \\
0, & \text { otherwise }
\end{array}, \forall i \in V, \quad \widehat{z}_{i}^{(E)}=\left\{\begin{array}{lc}
1, & i \in E_{\tau} \\
0, & \text { otherwise }
\end{array}, \forall j \in E .\right.\right.
$$

Clearly, $\widehat{\boldsymbol{z}}$ is a feasible solution to Model (30), with an objective value equal to $\frac{\tau(\tau+1)}{2}$. Thus, $R(\boldsymbol{Q}) \geq \frac{\tau(\tau+1)}{2}$.

Now suppose that $R(\boldsymbol{Q})>\frac{\tau(\tau+1)}{2}$. According to the objective function (30a), we must have

$$
\left|E^{*}\right|+\left|V^{*}\right|=\sum_{i \in E}\left(z_{i}^{(E)}\right)^{*}+\sum_{j \in V}\left(z_{j}^{(V)}\right)^{*} \geq R(\boldsymbol{Q})>\frac{\tau(\tau+1)}{2} .
$$

Also, the constraint $30 \mathrm{~b}$ implies that

$$
\left|E^{*}\right|+\left|V^{*}\right| \leq \frac{\tau(\tau+1)}{2},
$$

a contradiction.

"if". Suppose that $R(\boldsymbol{Q})=\frac{\tau(\tau+1)}{2}$. According to (31), we must have

$$
\frac{\tau(\tau+1)}{2}=R(\boldsymbol{Q})=\sum_{i \in E^{*}}\left(1-\sum_{j \in V \backslash V^{*}} \underline{\alpha}_{j i}\right)_{+}+\left|V^{*}\right| \leq\left|E^{*}\right|+\left|V^{*}\right|
$$

and by (32), we also have

$$
\frac{\tau(\tau+1)}{2}=R(\boldsymbol{Q}) \leq\left(\begin{array}{c}
\left|V^{*}\right| \\
2
\end{array}\right)+\left|V^{*}\right|
$$

On the other hand, the constraint (30b) implies that $\left|V^{*}\right|+\left|E^{*}\right| \leq \frac{\tau(\tau+1)}{2}$. Thus, we must have $\left|V^{*}\right|+\left|E^{*}\right|=\frac{\tau(\tau+1)}{2}$. Suppose that the substructure $\widehat{G}\left(V^{*}, E^{*}\right)$ is not a clique, 
then there exists $i_{0}=\left(u_{0}, v_{0}\right) \in E^{*}$ such that at least one of its nodes is not chosen, i.e., $\left(1-\sum_{j \in V \backslash V^{*}} \underline{\alpha}_{j i_{0}}\right)_{+}=0$. Thus, by (31), we have

$$
\frac{\tau(\tau+1)}{2}=R(\boldsymbol{Q})=\sum_{i \in E^{*} \backslash\left\{i_{0}\right\}}\left(1-\sum_{j \in V \backslash V^{*}} \underline{\alpha}_{j i}\right)_{+}+\left|V^{*}\right| \leq\left|E^{*}\right|-1+\left|V^{*}\right|<\frac{\tau(\tau+1)}{2}
$$

a contradiction.

\section{A.3 Proof of Theorem 2}

Theorem 2. Suppose $n=2, k=1$, and Assumption 2 holds, then the optimal order quantities $\boldsymbol{Q}^{*}=\left(Q_{1}^{*}, Q_{2}^{*}\right)$ are characterized by the following three cases:

Case 1: If $\bar{P}_{1} \leq \bar{P}_{2} \underline{\alpha}_{12}$ and $\bar{P}_{2} \geq \bar{P}_{1} \underline{\alpha}_{21}$, then $\left(Q_{1}^{*}, Q_{2}^{*}\right)=\left(0, D_{2}-l_{2}+\underline{\alpha}_{12} D_{1}\right)$.

Case 2: If $\bar{P}_{2} \leq \bar{P}_{1} \underline{\alpha}_{21}$ and $\bar{P}_{1} \geq \bar{P}_{2} \underline{\alpha}_{12}$, then $\left(Q_{1}^{*}, Q_{2}^{*}\right)=\left(D_{1}-l_{1}+\underline{\alpha}_{21} D_{2}, 0\right)$.

Case 3: If $\bar{P}_{1} \geq \bar{P}_{2} \underline{\alpha}_{12}$ and $\bar{P}_{2} \geq \bar{P}_{1} \underline{\alpha}_{21}$, then we have the following two sub-cases:

Sub-case 3.1: If $\bar{S}_{1} l_{1} \geq \bar{S}_{2} l_{2}$, then $\left(Q_{1}^{*}, Q_{2}^{*}\right)=\left(D_{1}-\frac{l_{1}-\underline{\alpha}_{21} l_{2}}{1-\underline{\alpha}_{12} \underline{\alpha}_{21}}, D_{2}-\frac{l_{2}-\underline{\alpha}_{12} l_{1}}{1-\underline{\alpha}_{12} \underline{\alpha}_{21}}\right)$ or $\left(Q_{1}^{*}, Q_{2}^{*}\right)=$ $\left(D_{1}, D_{2}-\frac{\bar{S}_{2} l_{2}-\bar{S}_{1} l_{1}}{\bar{S}_{2}-\bar{S}_{1} \underline{\alpha}_{21}}\right)$.

Sub-case 3.2: If $\bar{S}_{1} l_{1} \leq \bar{S}_{2} l_{2}$, then $\left(Q_{1}^{*}, Q_{2}^{*}\right)=\left(D_{1}-\frac{l_{1}-\underline{\alpha}_{21} l_{2}}{1-\underline{\alpha}_{12} \underline{\alpha}_{21}}, D_{2}-\frac{l_{2}-\underline{\alpha}_{12} l_{1}}{1-\underline{\alpha}_{12} \underline{\alpha}_{21}}\right)$ or $\left(Q_{1}^{*}, Q_{2}^{*}\right)=$ $\left(D_{1}-\frac{\bar{S}_{1} l_{1}-\bar{S}_{2} l_{2}}{\bar{S}_{1}-\bar{S}_{2} \underline{\alpha}_{12}}, D_{2}\right)$.

Proof. According to Model (14), we have

$$
v^{*}=\max _{Q_{1}, Q_{2} \geq 0}\left\{f(\boldsymbol{Q})=\bar{P}_{1} Q_{1}+\bar{P}_{2} Q_{2}-R(\boldsymbol{Q})\right\},
$$

and

$$
\begin{aligned}
R(\boldsymbol{Q})= & \max _{\boldsymbol{z} \in X} \sum_{i \in[2]} \bar{S}_{i}\left[Q_{i}-D_{i}+l_{i} z_{i}-\sum_{j \in[2]} \underline{\alpha}_{j i}\left(\left(D_{j}-l_{j}-Q_{j}\right)_{+} z_{j}+\left(D_{j}-Q_{j}\right)_{+}\left(1-z_{j}\right)\right)\right]_{+} \\
= & \max \left\{\bar{S}_{1}\left(Q_{1}-D_{1}+l_{1}-\underline{\alpha}_{21}\left(D_{2}-Q_{2}\right)_{+}\right)_{+}+\bar{S}_{2}\left(Q_{2}-D_{2}-\underline{\alpha}_{12}\left(D_{1}-l_{1}-Q_{1}\right)_{+}\right)_{+},\right. \\
& \bar{S}_{1}\left(Q_{1}-D_{1}-\underline{\alpha}_{21}\left(D_{2}-l_{2}-Q_{2}\right)_{+}\right)_{+}+\bar{S}_{2}\left(Q_{2}-D_{2}+l_{2}-\underline{\alpha}_{12}\left(D_{1}-Q_{1}\right)_{+}\right)_{+}, \\
& \left.\bar{S}_{1}\left(Q_{1}-D_{1}-\underline{\alpha}_{21}\left(D_{2}-l_{2}-Q_{2}\right)_{+}\right)_{+}+\bar{S}_{2}\left(Q_{2}-D_{2}-\underline{\alpha}_{12}\left(D_{1}-Q_{1}\right)_{+}\right)_{+}\right\} \\
= & \max \left\{\bar{S}_{1}\left(Q_{1}-D_{1}+l_{1}-\underline{\alpha}_{21}\left(D_{2}-Q_{2}\right)_{+}\right)_{+}+\bar{S}_{2}\left(Q_{2}-D_{2}-\underline{\alpha}_{12}\left(D_{1}-l_{1}-Q_{1}\right)_{+}\right)_{+},\right.
\end{aligned}
$$




$$
\left.\bar{S}_{1}\left(Q_{1}-D_{1}-\underline{\alpha}_{21}\left(D_{2}-l_{2}-Q_{2}\right)_{+}\right)_{+}+\bar{S}_{2}\left(Q_{2}-D_{2}+l_{2}-\underline{\alpha}_{12}\left(D_{1}-Q_{1}\right)_{+}\right)_{+}\right\},
$$

where the second equality is due to $X=\left\{\boldsymbol{z}: \sum_{i \in[2]} z_{i} \leq 1, z_{i} \in\{0,1\}, \forall i \in[2]\right\}=\{(0,1),(1,0),(0,0)\}$ and the third equality is because $\bar{S}_{1}\left(Q_{1}-D_{1}-\underline{\alpha}_{21}\left(D_{2}-l_{2}-Q_{2}\right)_{+}\right)_{+}+\bar{S}_{2}\left(Q_{2}-D_{2}-\underline{\alpha}_{12}\left(D_{1}-Q_{1}\right)_{+}\right)_{+}$ is dominated by the other two.

Note that for each $i \in[2]$, the optimal order quantity $Q_{i}^{*}$ must belong to one of the three intervals $\left[0, D_{i}-l_{i}\right],\left[D_{i}-l_{i}, D_{i}\right]$, or $\left[D_{i},+\infty\right)$. Thus, we can divide the feasible region into 9 subregions (see Figure 3 for an illustration), where under each subregion, function $R(\boldsymbol{Q})$ becomes piecewise convex, thus Model (33) is solvable. Therefore, we can optimize Model (33) over each subregion, and the solution with the largest objective value corresponds to an optimal solution to the original problem (33). Therefore, we need to discuss the 9 cases, corresponding to 9 subregions.

Before we derive the main results, we observe a characterization of an optimal solution of maximizing a piecewise concave function over a box.

Observation 1. Given an integer number $\tau$, consider the following piecewise concave optimization program:

$$
\max _{\boldsymbol{x}}\left\{\min _{i \in[\tau]}\left(\boldsymbol{c}^{i}\right)^{\top} \boldsymbol{x}: \mathbf{0} \leq \boldsymbol{x} \leq \boldsymbol{U}\right\} .
$$

Then an optimal solution of the above optimization problem can be one of the following points:

(i) the extreme points of the box $[\mathbf{0}, \boldsymbol{U}]$; and

(ii) the intersection point of any affine system $\left(\boldsymbol{c}^{i}\right)^{\top} \boldsymbol{x}=\left(\boldsymbol{c}^{j}\right)^{\top} \boldsymbol{x}$ for all $i, j \in \mathbb{B} \subseteq[\tau]$ with $2 \leq|\mathbb{B}| \leq n$ and the boundary of the box $[\mathbf{0}, \boldsymbol{U}]$.

(iii) the unique solution of the affine system $\left(\boldsymbol{c}^{i}\right)^{\top} \boldsymbol{x}=\left(\boldsymbol{c}^{j}\right)^{\top} \boldsymbol{x}$ for all $i, j \in \mathbb{B}$ with $|\mathbb{B}|=n+1$, which is in the box $[\mathbf{0}, \boldsymbol{U}]$.

Proof. Note that the piecewise concave optimization program can be written as the following linear program:

$$
\max _{\boldsymbol{x}}\left\{w: w \leq\left(\boldsymbol{c}^{i}\right)^{\top} \boldsymbol{x}, \forall i \in[\tau], \mathbf{0} \leq \boldsymbol{x} \leq \boldsymbol{U}\right\} .
$$

The conclusion follows by the fact that one optimal solution of the above linear program must be an extreme point, and condition (i), (ii), and (iii) exactly characterize all the extreme points.

Now we are ready to discuss the following 9 cases.

Case 1. Suppose $\left(Q_{1}, Q_{2}\right) \in \Omega_{1}$, i.e., $0 \leq Q_{1} \leq D_{1}-l_{1}, 0 \leq Q_{2} \leq D_{2}-l_{2}$.

In this case, $R(\boldsymbol{Q})=0$ and Model $(33)$ becomes:

$$
\max _{\boldsymbol{Q}}\left\{f(\boldsymbol{Q})=\bar{P}_{1} Q_{1}+\bar{P}_{2} Q_{2}: 0 \leq Q_{1} \leq D_{1}-l_{1}, 0 \leq Q_{2} \leq D_{2}-l_{2}\right\}
$$




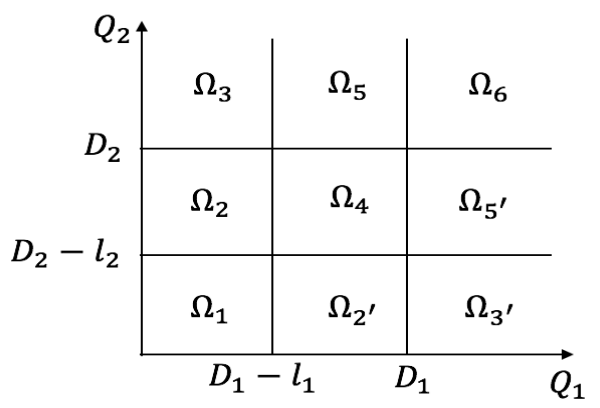

Figure 3: Decomposition of feasible solution regions into 9 sub-regions, where $\Omega_{1}=$ $\left\{\left(Q_{1}, Q_{2}\right): 0 \leq Q_{1} \leq D_{1}-l_{1}, 0 \leq Q_{2} \leq D_{2}-l_{2}\right\}, \quad \Omega_{2} \quad=\quad\left\{\left(Q_{1}, Q_{2}\right): 0 \leq Q_{1} \leq D_{1}-l_{1}, D_{2}-l_{2} \leq Q_{2} \leq D_{2}\right\}$, $\Omega_{3}=\left\{\left(Q_{1}, Q_{2}\right): 0 \leq Q_{1} \leq D_{1}-l_{1}, D_{2} \leq Q_{2}\right\}, \quad \Omega_{4}=\left\{\left(Q_{1}, Q_{2}\right): D_{1}-l_{1} \leq Q_{1} \leq D_{1}, D_{2}-l_{2} \leq Q_{2} \leq D_{2}\right\}$, $\Omega_{5}=\left\{\left(Q_{1}, Q_{2}\right): D_{1}-l_{1} \leq Q_{1} \leq D_{1}, D_{2} \leq Q_{2}\right\}, \quad \Omega_{6} \quad=\quad\left\{\left(Q_{1}, Q_{2}\right): D_{1} \leq Q_{1}, D_{2} \leq Q_{2}\right\}, \quad \Omega_{2^{\prime}}=$ $\left\{\left(Q_{1}, Q_{2}\right): D_{1}-l_{1} \leq Q_{1} \leq D_{1}, 0 \leq Q_{2} \leq D_{2}-l_{2}\right\}, \quad \Omega_{3^{\prime}} \quad=\quad\left\{\left(Q_{1}, Q_{2}\right): D_{1} \leq Q_{1}, 0 \leq Q_{2} \leq D_{2}-l_{2}\right\}, \quad \Omega_{5^{\prime}} \quad=$ $\left\{\left(Q_{1}, Q_{2}\right): D_{1} \leq Q_{1}, D_{2}-l_{2} \leq Q_{2} \leq D_{2}\right\}$.

Clearly, the optimal solution of the above linear program is $\boldsymbol{t}_{1}^{1}=\left(D_{1}-l_{1}, D_{2}-l_{2}\right)$, and its optimal total profit is

$$
f\left(\boldsymbol{t}_{1}^{1}\right)=\bar{P}_{1}\left(D_{1}-l_{1}\right)+\bar{P}_{2}\left(D_{2}-l_{2}\right) .
$$

Case 2. Suppose $\left(Q_{1}, Q_{2}\right) \in \Omega_{2}$, i.e., $0 \leq Q_{1} \leq D_{1}-l_{1}, D_{2}-l_{2} \leq Q_{2} \leq D_{2}$.

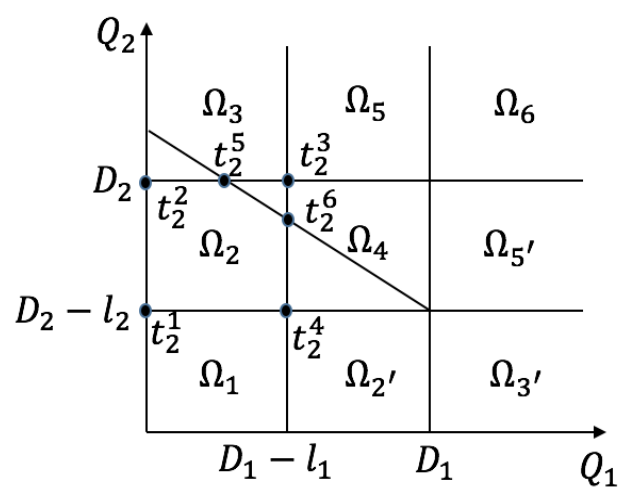

Figure 4: Illustration of possible solutions of Case 2.

In this case, we have $R(\boldsymbol{Q})=\bar{S}_{2}\left(Q_{2}-D_{2}+l_{2}-\underline{\alpha}_{12}\left(D_{1}-Q_{1}\right)\right)_{+}$and Model (33) becomes:

$$
\max _{\boldsymbol{Q}}\left\{f(\boldsymbol{Q})=\bar{P}_{1} Q_{1}+\bar{P}_{2} Q_{2}-\bar{S}_{2}\left(Q_{2}-D_{2}+l_{2}-\underline{\alpha}_{12}\left(D_{1}-Q_{1}\right)\right)_{+}: 0 \leq Q_{1} \leq D_{1}-l_{1}, D_{2}-l_{2} \leq Q_{2} \leq D_{2}\right\} .
$$

According to Observation 1, the optimal solution of above optimization problem can be one of the following points: (1) extreme points of $\Omega_{2}$ i.e., $\boldsymbol{t}_{2}^{1}, \boldsymbol{t}_{2}^{2}, \boldsymbol{t}_{2}^{3}, \boldsymbol{t}_{2}^{4}$; and (2) the intersection points of linear equation $Q_{2}-D_{2}+l_{2}-\underline{\alpha}_{12}\left(D_{1}-Q_{1}\right)=0$ with feasible regions, i.e., $\boldsymbol{t}_{2}^{5}, \boldsymbol{t}_{2}^{6}$ (see Figure 4 for an illustration). These potential optimal solutions and their corresponding total profits are listed 
in Table 4.

Table 4: The possible solutions and their total profits in Case 2

\begin{tabular}{|c|c|}
\hline Possible solutions & Total profit \\
\hline $\boldsymbol{t}_{2}^{1}=\left(0, D_{2}-l_{2}\right)$ & $f\left(\boldsymbol{t}_{2}^{1}\right)=\bar{P}_{2}\left(D_{2}-l_{2}\right)$ \\
\hline $\boldsymbol{t}_{2}^{2}=\left(0, D_{2}\right)$ & $f\left(\boldsymbol{t}_{2}^{2}\right)=\bar{P}_{2} D_{2}$ \\
\hline $\boldsymbol{t}_{2}^{3}=\left(D_{1}-l_{1}, D_{2}\right)$ & $f\left(\boldsymbol{t}_{2}^{3}\right)=\bar{P}_{1}\left(D_{1}-l_{1}\right)+\bar{P}_{2} D_{2}-\bar{S}_{2}\left(l_{2}-\underline{\alpha}_{12} l_{1}\right)$ \\
\hline $\boldsymbol{t}_{1}^{1}=\boldsymbol{t}_{2}^{4}=\left(D_{1}-l_{1}, D_{2}-l_{2}\right)$ & $f\left(\boldsymbol{t}_{2}^{4}\right)=f\left(\boldsymbol{t}_{1}^{1}\right)=\bar{P}_{1}\left(D_{1}-l_{1}\right)+\bar{P}_{2}\left(D_{2}-l_{2}\right)$ \\
\hline $\boldsymbol{t}_{2}^{5}=\left(D_{1}-l_{2} / \underline{\alpha}_{12}, D_{2}\right)$ & $f\left(\boldsymbol{t}_{2}^{5}\right)=\bar{P}_{1}\left(D_{1}-l_{2} / \underline{\alpha}_{12}\right)+\bar{P}_{2} D_{2}$ \\
\hline $\boldsymbol{t}_{2}^{6}=\left(D_{1}-l_{1}, D_{2}-l_{2}+\underline{\alpha}_{12} l_{1}\right)$ & $f\left(\boldsymbol{t}_{2}^{6}\right)=\bar{P}_{1}\left(D_{1}-l_{1}\right)+\bar{P}_{2}\left(D_{2}-l_{2}+\underline{\alpha}_{12} l_{1}\right)$ \\
\hline
\end{tabular}

It remains to compare these solutions. Clearly, we have

- $f\left(\boldsymbol{t}_{2}^{2}\right) \geq f\left(\boldsymbol{t}_{2}^{1}\right)$, since $\bar{P}_{2}, l_{2} \geq 0$,

- $f\left(\boldsymbol{t}_{2}^{5}\right)-f\left(\boldsymbol{t}_{2}^{2}\right)=\bar{P}_{1}\left(D_{1}-l_{2} / \underline{\alpha}_{12}\right) \geq 0$ due to Assumption 2 that $\underline{\alpha}_{12} D_{1}-l_{2} \geq 0$,

- $f\left(\boldsymbol{t}_{2}^{6}\right)-f\left(\boldsymbol{t}_{2}^{3}\right)=\bar{P}_{2}\left(-l_{2}+\underline{\alpha}_{12} l_{1}\right)+\bar{S}_{2}\left(l_{2}-\alpha_{12} l_{1}\right)=\left(-\bar{P}_{2}+\bar{S}_{2}\right)\left(l_{2}-\underline{\alpha}_{12} l_{1}\right) \geq 0$ due to $\bar{S}_{2} \geq \bar{P}_{2}$ and Assumption 2 that $l_{2}-\underline{\alpha}_{12} l_{1} \geq 0$,

- $f\left(\boldsymbol{t}_{2}^{6}\right)-f\left(\boldsymbol{t}_{2}^{4}\right)=\bar{P}_{2} \underline{\alpha}_{12} l_{1} \geq 0$, and

- $f\left(\boldsymbol{t}_{2}^{6}\right)-f\left(\boldsymbol{t}_{2}^{5}\right)=-\bar{P}_{1} l_{1}+\bar{P}_{2}\left(-l_{2}+\underline{\alpha}_{12} l_{1}\right)+\bar{P}_{1} \frac{l_{2}}{\underline{\alpha}_{12}}=\frac{1}{\underline{\alpha}_{12}}\left(\bar{P}_{1}-\bar{P}_{2} \underline{\alpha}_{12}\right)\left(l_{2}-\underline{\alpha}_{12} l_{1}\right)\left\{\begin{array}{ll}\geq 0, & \text { if } \bar{P}_{1} \geq \bar{P}_{2} \underline{\alpha}_{12} \\ \leq 0, & \text { if } \bar{P}_{1} \leq \bar{P}_{2} \underline{\alpha}_{12}\end{array}\right.$, due to Assumption 2 that $l_{2}-\underline{\alpha}_{12} l_{1} \geq 0$.

From the above comparison, we can draw the following conclusion on the best solution in the subregions $\Omega_{1}$ and $\Omega_{2}$ :

(i) If $\bar{P}_{1}-\bar{P}_{2} \underline{\alpha}_{12} \leq 0$, then the point $\boldsymbol{t}_{2}^{5}$ dominates the other points in the subregions $\Omega_{1}$ and $\Omega_{2}$, since $f\left(\boldsymbol{t}_{2}^{5}\right) \geq f\left(\boldsymbol{t}_{2}^{2}\right) \geq f\left(\boldsymbol{t}_{2}^{1}\right), f\left(\boldsymbol{t}_{2}^{5}\right) \geq f\left(\boldsymbol{t}_{2}^{6}\right) \geq f\left(\boldsymbol{t}_{2}^{4}\right)$, and $f\left(\boldsymbol{t}_{2}^{5}\right) \geq f\left(\boldsymbol{t}_{2}^{6}\right) \geq f\left(\boldsymbol{t}_{2}^{3}\right)$.

(ii) If $\bar{P}_{1}-\bar{P}_{2} \underline{\alpha}_{12} \geq 0$, then the point $\boldsymbol{t}_{2}^{6}$ dominates the other points in the subregions $\Omega_{1}$ and $\Omega_{2}$, since $f\left(\boldsymbol{t}_{2}^{6}\right) \geq f\left(\boldsymbol{t}_{2}^{5}\right) \geq f\left(\boldsymbol{t}_{2}^{2}\right) \geq f\left(\boldsymbol{t}_{2}^{1}\right), f\left(\boldsymbol{t}_{2}^{6}\right) \geq f\left(\boldsymbol{t}_{2}^{4}\right)$, and $f\left(\boldsymbol{t}_{2}^{6}\right) \geq f\left(\boldsymbol{t}_{2}^{3}\right)$.

Case 3. Suppose $\left(Q_{1}, Q_{2}\right) \in \Omega_{3}$, i.e., $0 \leq Q_{1} \leq D_{1}-l_{1}, D_{2} \leq Q_{2}$.

In this case, $R(\boldsymbol{Q})=\bar{S}_{2}\left(Q_{2}-D_{2}+l_{2}-\underline{\alpha}_{12}\left(D_{1}-Q_{1}\right)\right)_{+}$and Model 33 becomes:

$\max _{\boldsymbol{Q}}\left\{f(\boldsymbol{Q})=\bar{P}_{1} Q_{1}+\bar{P}_{2} Q_{2}-\bar{S}_{2}\left(Q_{2}-D_{2}+l_{2}-\underline{\alpha}_{12}\left(D_{1}-Q_{1}\right)\right)_{+}: 0 \leq Q_{1} \leq D_{1}-l_{1}, D_{2} \leq Q_{2}\right\}$ 
According to Observation 1, the optimal solution can be one of the following points: (1) the extreme points in $\Omega_{3}$, i.e., $\boldsymbol{t}_{3}^{1}, \boldsymbol{t}_{3}^{2}$; and (2) the intersection points of linear $Q_{2}-D_{2}+l_{2}-\underline{\alpha}_{12}\left(D_{1}-Q_{1}\right)=$ 0 and the boundary of $\Omega_{3}$, i.e., $\boldsymbol{t}_{3}^{3}, \boldsymbol{t}_{3}^{4}$ (see Figure 5 for an illustration). These solutions and their corresponding total profits are listed in Table 5 .

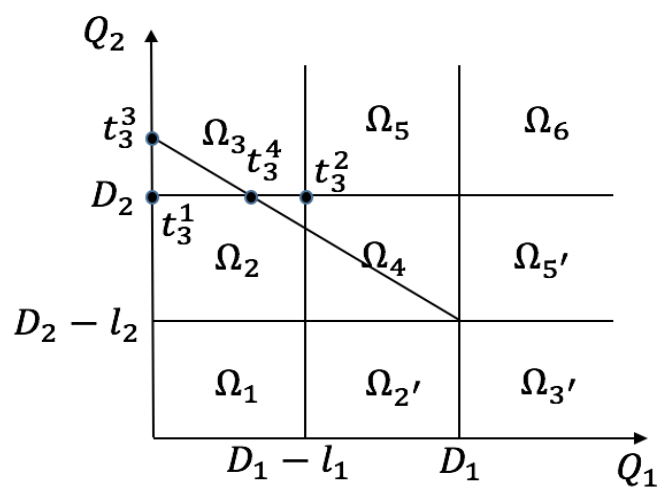

Figure 5: Possible solutions in Case 3

Table 5: Possible solutions and their total profits in Case 3

\begin{tabular}{|c|c|}
\hline Possible solutions & Total profit \\
\hline $\boldsymbol{t}_{3}^{1}=\boldsymbol{t}_{2}^{2}=\left(0, D_{2}\right)$ & $f\left(\boldsymbol{t}_{3}^{1}\right)=f\left(\boldsymbol{t}_{2}^{2}\right)=\bar{P}_{2} D_{2}$ \\
\hline $\boldsymbol{t}_{3}^{2}=\boldsymbol{t}_{2}^{3}=\left(D_{1}-l_{1}, D_{2}\right)$ & $f\left(\boldsymbol{t}_{3}^{2}\right)=f\left(\boldsymbol{t}_{2}^{3}\right)=\bar{P}_{1}\left(D_{1}-l_{1}\right)+\bar{P}_{2} D_{2}-\bar{S}_{2}\left(l_{2}-\underline{\alpha}_{12} l_{1}\right)$ \\
\hline $\boldsymbol{t}_{3}^{3}=\left(0, D_{2}-l_{2}+\underline{\alpha}_{12} D_{1}\right)$ & $f\left(\boldsymbol{t}_{3}^{3}\right)=\bar{P}_{2}\left(D_{2}-l_{2}+\underline{\alpha}_{12} D_{1}\right)$ \\
\hline $\boldsymbol{t}_{3}^{4}=\boldsymbol{t}_{2}^{5}=\left(D_{1}-l_{2} / \underline{\alpha}_{12}, D_{2}\right)$ & $f\left(\boldsymbol{t}_{3}^{4}\right)=f\left(\boldsymbol{t}_{2}^{5}\right)=\bar{P}_{1}\left(D_{1}-l_{2} / \underline{\alpha}_{12}\right)+\bar{P}_{2} D_{2}$ \\
\hline
\end{tabular}

In view of the results in Case 1 and Case 2, it remains to compare $f\left(\boldsymbol{t}_{2}^{5}\right), f\left(\boldsymbol{t}_{3}^{3}\right)$ and also $f\left(\boldsymbol{t}_{2}^{6}\right), f\left(\boldsymbol{t}_{3}^{3}\right)$. Clearly, we have

- $f\left(\boldsymbol{t}_{2}^{5}\right)-f\left(\boldsymbol{t}_{3}^{3}\right)=\bar{P}_{1}\left(D_{1}-l_{2} / \underline{\alpha}_{12}\right)-\bar{P}_{2}\left(-l_{2}+\underline{\alpha}_{12} D_{1}\right)=\frac{1}{\underline{\alpha}_{12}}\left(\bar{P}_{1}-\bar{P}_{2} \underline{\alpha}_{12}\right)\left(\underline{\alpha}_{12} D_{1}-l_{2}\right)\left\{\begin{array}{ll}\geq 0, & \text { if } \bar{P}_{1} \geq \bar{P}_{2} \underline{\alpha}_{12} \\ \leq 0, & \text { if } \bar{P}_{1} \leq \bar{P}_{2} \underline{\alpha}_{12}\end{array}\right.$, due to Assumption 2 that $\underline{\alpha}_{12} D_{1} \geq l_{2}$,

- $f\left(\boldsymbol{t}_{2}^{6}\right)-f\left(\boldsymbol{t}_{3}^{3}\right)=\bar{P}_{1}\left(D_{1}-l_{1}\right)+\bar{P}_{2} \underline{\alpha}_{12}\left(l_{1}-D_{1}\right)=\left(\bar{P}_{1}-\bar{P}_{2} \underline{\alpha}_{12}\right)\left(D_{1}-l_{1}\right) \begin{cases}\geq 0, & \text { if } \bar{P}_{1} \geq \bar{P}_{2} \underline{\alpha}_{12} \\ \leq 0, & \text { if } \bar{P}_{1} \leq \bar{P}_{2} \underline{\alpha}_{12}\end{cases}$ since $D_{l} \geq l_{1}$,

From the above comparison as well as the results of Case 1 and Case 2, we can draw the following conclusion on the best solution in subregions $\Omega_{1}, \Omega_{2}$ and $\Omega_{3}$ : 
(i) If $\bar{P}_{1} \geq \bar{P}_{2} \underline{\alpha}_{12}$, then $\boldsymbol{t}_{2}^{6}$ dominates all the other points in $\Omega_{1}, \Omega_{2}$, and $\Omega_{3}$ since $f\left(\boldsymbol{t}_{2}^{6}\right) \geq f\left(\boldsymbol{t}_{2}^{5}\right) \geq$ $f\left(\boldsymbol{t}_{3}^{3}\right)$.

(ii) If $\bar{P}_{1} \leq \bar{P}_{2} \underline{\alpha}_{12}$, then $\boldsymbol{t}_{3}^{3}$ dominates all the other points in $\Omega_{1}, \Omega_{2}$, and $\Omega_{3}$ since $f\left(\boldsymbol{t}_{2}^{6}\right) \leq f\left(\boldsymbol{t}_{2}^{5}\right) \leq$ $f\left(\boldsymbol{t}_{3}^{3}\right)$.

Case 4. Suppose $\left(Q_{1}, Q_{2}\right) \in \Omega_{4}$, i.e., $D_{1}-l_{1} \leq Q_{1} \leq D_{1}, D_{2}-l_{2} \leq Q_{2} \leq D_{2}$.

In this case, $R(\boldsymbol{Q})=\max \left\{\bar{S}_{1}\left(Q_{1}-D_{1}+l_{1}-\underline{\alpha}_{21}\left(D_{2}-Q_{2}\right)\right)_{+}, \bar{S}_{2}\left(Q_{2}-D_{2}+l_{2}-\underline{\alpha}_{12}\left(D_{1}-Q_{1}\right)\right)_{+}\right\}$ and Model (33) becomes:

$$
\max _{\boldsymbol{Q}}\left\{f(\boldsymbol{Q})=\bar{P}_{1} Q_{1}+\bar{P}_{2} Q_{2}-R(\boldsymbol{Q}): D_{1}-l_{1} \leq Q_{1} \leq D_{1}, D_{2}-l_{2} \leq Q_{2} \leq D_{2}\right\} .
$$

According to Observation 1, the optimal solution can be one of the following points: (1) the extreme points of $\Omega_{4}$, i.e., $\boldsymbol{t}_{4}^{1}, \boldsymbol{t}_{4}^{2}, \boldsymbol{t}_{4}^{3}, \boldsymbol{t}_{4}^{4}$; (2) the intersection points of the line $Q_{1}-D_{1}+l_{1}-$ $\underline{\alpha}_{11}\left(D_{2}-Q_{2}\right)=0$ with the boundary of $\Omega_{4}$, i.e., $\boldsymbol{t}_{4}^{2}, \boldsymbol{t}_{4}^{5}$; (3) the intersection points of the line $Q_{2}-D_{2}+l_{2}-\underline{\alpha}_{12}\left(D_{1}-Q_{1}\right)=0$ with the boundary of $\Omega_{4}$, i.e., $\boldsymbol{t}_{4}^{4}, \boldsymbol{t}_{4}^{6}$; and (4) the intersection point of two lines $\bar{S}_{1}\left(Q_{1}-D_{1}+l_{1}-\underline{\alpha}_{21}\left(D_{2}-Q_{2}\right)\right)_{+}=0$ and $\bar{S}_{2}\left(Q_{2}-D_{2}+l_{2}-\underline{\alpha}_{12}\left(D_{1}-Q_{1}\right)\right)_{+}=0$, i.e., $\boldsymbol{t}_{4}^{7}$ (see Figure 6 for an illustration). These solutions and their total profits are listed in Table 6 .

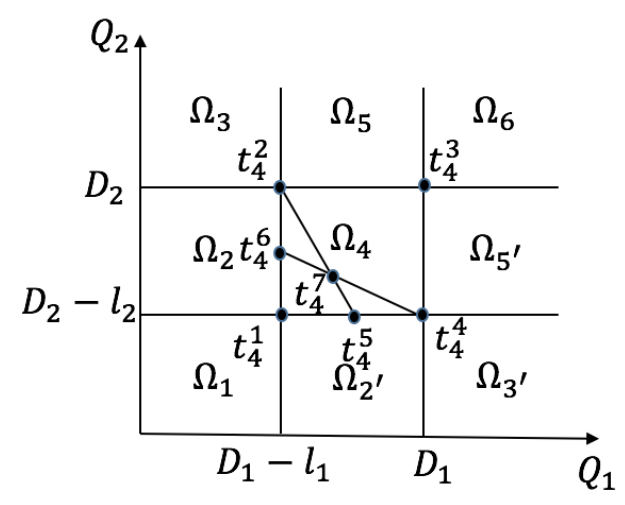

Figure 6: Possible solutions in Case 4 
Table 6: Possible solutions and their total profits in Case 4

\begin{tabular}{|c|c|}
\hline Possible solutions & Total profit \\
\hline $\boldsymbol{t}_{4}^{1}=\boldsymbol{t}_{2}^{4}=\boldsymbol{t}_{1}^{1}=\left(D_{1}-l_{1}, D_{2}-l_{2}\right)$ & $f\left(\boldsymbol{t}_{4}^{1}\right)=f\left(\boldsymbol{t}_{2}^{4}\right)=f\left(\boldsymbol{t}_{1}^{1}\right)=\bar{P}_{1}\left(D_{1}-l_{1}\right)+\bar{P}_{2}\left(D_{2}-l_{2}\right)$ \\
\hline $\boldsymbol{t}_{4}^{2}=\boldsymbol{t}_{3}^{2}=\boldsymbol{t}_{2}^{3}=\left(D_{1}-l_{1}, D_{2}\right)$ & $f\left(\boldsymbol{t}_{4}^{2}\right)=f\left(\boldsymbol{t}_{3}^{2}\right)=f\left(\boldsymbol{t}_{2}^{3}\right)=\bar{P}_{1}\left(D_{1}-l_{1}\right)+\bar{P}_{2} D_{2}-\bar{S}_{2}\left(l_{2}-\underline{\alpha}_{12} l_{1}\right)$ \\
\hline $\boldsymbol{t}_{4}^{3}=\left(D_{1}, D_{2}\right)$ & $f\left(\boldsymbol{t}_{4}^{3}\right)=\bar{P}_{1} D_{1}+\bar{P}_{2} D_{2}-\max \left\{\bar{S}_{1} l_{1}, \bar{S}_{2} l_{2}\right\}$ \\
\hline $\boldsymbol{t}_{4}^{4}=\left(D_{1}, D_{2}-l_{2}\right)$ & $f\left(\boldsymbol{t}_{4}^{4}\right)=\bar{P}_{1} D_{1}+\bar{P}_{2}\left(D_{2}-l_{2}\right)-\bar{S}_{1}\left(l_{1}-\underline{\alpha}_{21} l_{2}\right)$ \\
\hline $\boldsymbol{t}_{4}^{5}=\left(D_{1}-l_{1}+\underline{\alpha}_{21} l_{2}, D_{2}-l_{2}\right)$ & $f\left(\boldsymbol{t}_{4}^{5}\right)=\bar{P}_{1}\left(D_{1}-l_{1}+\underline{\alpha}_{21} l_{2}\right)+\bar{P}_{2}\left(D_{2}-l_{2}\right)$ \\
\hline $\boldsymbol{t}_{4}^{6}=\boldsymbol{t}_{2}^{6}=\left(D_{1}-l_{1}, D_{2}-l_{2}+\underline{\alpha}_{12} l_{1}\right)$ & $f\left(\boldsymbol{t}_{4}^{6}\right)=f\left(\boldsymbol{t}_{2}^{6}\right)=\bar{P}_{1}\left(D_{1}-l_{1}\right)+\bar{P}_{2}\left(D_{2}-l_{2}+\underline{\alpha}_{12} l_{1}\right)$ \\
\hline $\boldsymbol{t}_{4}^{7}=\left(D_{1}-\frac{l_{1}-\underline{\alpha}_{21} l_{2}}{1-\underline{\alpha}_{12} \underline{\alpha}_{21}}, D_{2}-\frac{l_{2}-\underline{\alpha}_{12} l_{1}}{1-\underline{\alpha}_{12} \underline{\alpha}_{21}}\right)$ & $f\left(\boldsymbol{t}_{4}^{7}\right)=\bar{P}_{1}\left(D_{1}-\frac{l_{1}-\underline{\alpha}_{21} l_{2}}{1-\underline{\alpha}_{12} \underline{\alpha}_{21}}\right)+\bar{P}_{2}\left(D_{2}-\frac{l_{2}-\underline{\alpha}_{12} l_{1}}{1-\underline{\alpha}_{12} \underline{\alpha}_{21}}\right)$ \\
\hline
\end{tabular}

In view of the results in Case 1- Case 3, we know that point $\boldsymbol{t}_{2}^{6}$ or $\boldsymbol{t}_{3}^{3}$ dominates all the other points of $\Omega_{1}, \Omega_{2}$, and $\Omega_{3}$, so we only need to compare $f\left(\boldsymbol{t}_{2}^{6}\right), f\left(\boldsymbol{t}_{3}^{3}\right), f\left(\boldsymbol{t}_{4}^{3}\right), f\left(\boldsymbol{t}_{4}^{4}\right), f\left(\boldsymbol{t}_{4}^{5}\right), f\left(\boldsymbol{t}_{4}^{7}\right)$.

- $f\left(\boldsymbol{t}_{4}^{4}\right)-f\left(\boldsymbol{t}_{4}^{5}\right)=\left(\bar{P}_{1}-\bar{S}_{1}\right)\left(l_{1}-\underline{\alpha}_{21} l_{2}\right) \leq 0$ due to Assumption 2 that $l_{1}-\underline{\alpha}_{21} l_{2} \geq 0$ and the fact that $\bar{P}_{1} \leq \bar{S}_{1}$,

- If $\bar{P}_{1} \leq \bar{P}_{2} \underline{\alpha}_{12}$, then

$$
\begin{aligned}
f\left(\boldsymbol{t}_{4}^{3}\right)-f\left(\boldsymbol{t}_{3}^{3}\right) & =\bar{P}_{1} D_{1}-\max \left\{\bar{S}_{1} l_{1}, \bar{S}_{2} l_{2}\right\}+\bar{P}_{2} l_{2}-\bar{P}_{2} \underline{\alpha}_{12} D_{1} \\
& =\left(\bar{P}_{1}-\bar{P}_{2} \underline{\alpha}_{12}\right) D_{1}+\left(\bar{P}_{2} l_{2}-\max \left\{\bar{S}_{1} l_{1}, \bar{S}_{2} l_{2}\right\}\right) \leq 0,
\end{aligned}
$$

where the inequality is because of $D_{1} \geq 0$ and $\bar{P}_{2} l_{2} \leq \bar{S}_{2} l_{2} \leq \max \left\{\bar{S}_{1} l_{1}, \bar{S}_{2} l_{2}\right\}$. Otherwise, $f\left(\boldsymbol{t}_{4}^{3}\right)$ and $f\left(\boldsymbol{t}_{3}^{3}\right)$ are incomparable.

- Compare $f\left(\boldsymbol{t}_{2}^{6}\right)$ with $f\left(\boldsymbol{t}_{4}^{7}\right)$

$$
\begin{aligned}
f\left(\boldsymbol{t}_{2}^{6}\right)-f\left(\boldsymbol{t}_{4}^{7}\right) & =\bar{P}_{2}\left(-l_{2}+\underline{\alpha}_{12} l_{1}\right)+\bar{P}_{1}\left(-l_{1}\right)+\bar{P}_{1} \frac{l_{1}-\underline{\alpha}_{21} l_{2}}{1-\underline{\alpha}_{12} \underline{\alpha}_{21}}+\bar{P}_{2} \frac{l_{2}-\underline{\alpha}_{12} l_{1}}{1-\underline{\alpha}_{12} \underline{\alpha}_{21}} \\
& =-\underline{\alpha}_{21} \frac{\left(\bar{P}_{1}-\bar{P}_{2} \underline{\alpha}_{12}\right)\left(l_{2}-l_{1} \underline{\alpha}_{12}\right)}{1-\underline{\alpha}_{21} \underline{\alpha}_{21}}\left\{\begin{array}{l}
\leq 0, \quad \text { if } \bar{P}_{1} \geq \bar{P}_{2} \underline{\alpha}_{12} \\
\geq 0, \quad \text { if } \bar{P}_{1} \leq \bar{P}_{2} \underline{\alpha}_{12}
\end{array}\right.
\end{aligned}
$$

where the inequalities are due to Assumption 2 that $l_{2} \geq \underline{\alpha}_{12} l_{1}$ and the fact that $0 \leq \underline{\alpha}_{21} \leq$ $1,0 \leq \underline{\alpha}_{12} \leq 1$.

- If $\bar{P}_{1} \leq \bar{P}_{2} \underline{\alpha}_{12}$, then

$$
f\left(\boldsymbol{t}_{4}^{5}\right)-f\left(\boldsymbol{t}_{3}^{3}\right)=\bar{P}_{1}\left(D_{1}-l_{1}+\underline{\alpha}_{21} l_{2}\right)-\bar{P}_{2} \underline{\alpha}_{12} D_{1}=\left(\bar{P}_{1}-\bar{P}_{2} \underline{\alpha}_{12}\right) D_{1}-\bar{P}_{1}\left(l_{1}-\underline{\alpha}_{21} l_{2}\right) \leq 0,
$$

where the inequality is due to Assumption 2 that $l_{1} \geq \underline{\alpha}_{21} l_{2}$ and the fact that $\bar{P}_{1} \geq 0, D_{1} \geq 0$. 
- Compare $f\left(\boldsymbol{t}_{2}^{6}\right)$ with $f\left(\boldsymbol{t}_{3}^{3}\right)$

$$
\begin{aligned}
& f\left(\boldsymbol{t}_{2}^{6}\right)-f\left(\boldsymbol{t}_{3}^{3}\right)=\bar{P}_{1}\left(D_{1}-l_{1}\right)+\bar{P}_{2} \underline{\alpha}_{12} l_{1}-\bar{P}_{2} \underline{\alpha}_{12} D_{1}=\left(\bar{P}_{1}-\bar{P}_{2} \underline{\alpha}_{12}\right)\left(D_{1}-l_{1}\right) \\
& \left\{\begin{array}{ll}
\geq 0, & \text { if } \bar{P}_{1} \geq \bar{P}_{2} \underline{\alpha}_{12} \\
\leq 0, & \text { if } \bar{P}_{1} \leq \bar{P}_{2} \underline{\alpha}_{12}
\end{array},\right.
\end{aligned}
$$

where the inequalities are due to the fact that $D_{1} \geq l_{1}$.

- Compare $f\left(\boldsymbol{t}_{4}^{5}\right)$ with $f\left(\boldsymbol{t}_{4}^{7}\right)$

$$
\begin{aligned}
f\left(\boldsymbol{t}_{4}^{5}\right)-f\left(\boldsymbol{t}_{4}^{7}\right) & =\bar{P}_{1}\left(-l_{1}+\underline{\alpha}_{21} l_{2}\right)+\bar{P}_{2}\left(-l_{2}\right)+\bar{P}_{1} \frac{l_{1}-\underline{\alpha}_{21} l_{2}}{1-\underline{\alpha}_{12} \underline{\alpha}_{21}}+\bar{P}_{2} \frac{l_{2}-\underline{\alpha}_{12} l_{1}}{1-\underline{\alpha}_{12} \underline{\alpha}_{21}} \\
& =-\underline{\alpha}_{12} \frac{\left(\bar{P}_{2}-\bar{P}_{1} \underline{\alpha}_{21}\right)\left(l_{1}-l_{2} \underline{\alpha}_{21}\right)}{1-\underline{\alpha}_{12} \underline{\alpha}_{21}}\left\{\begin{array}{l}
\leq 0, \quad \text { if } \bar{P}_{2} \geq \bar{P}_{1} \underline{\alpha}_{21} \\
\geq 0, \quad \text { if } \bar{P}_{2} \leq \bar{P}_{1} \underline{\alpha}_{21}
\end{array}\right.
\end{aligned}
$$

where the inequalities are due to Assumption 2 that $l_{1} \geq \underline{\alpha}_{21} l_{2}$ and the fact that $0 \leq \underline{\alpha}_{21} \leq$ $1,0 \leq \underline{\alpha}_{12} \leq 1$.

- Compare $f\left(\boldsymbol{t}_{2}^{5}\right)$ with $f\left(\boldsymbol{t}_{4}^{7}\right)$

$$
\begin{aligned}
f\left(\boldsymbol{t}_{2}^{5}\right)-f\left(\boldsymbol{t}_{4}^{7}\right) & =-\bar{P}_{1} \frac{l_{2}}{\underline{\alpha}_{12}}+\bar{P}_{1} \frac{l_{1}-\underline{\alpha}_{21} l_{2}}{1-\underline{\alpha}_{12} \underline{\alpha}_{21}}+\bar{P}_{2} \frac{l_{2}-\underline{\alpha}_{12} l_{1}}{1-\underline{\alpha}_{12} \underline{\alpha}_{21}}=-\frac{\left(\bar{P}_{1}-\bar{P}_{2} \underline{\alpha}_{12}\right)\left(l_{2}-l_{1} \underline{\alpha}_{12}\right)}{\underline{\alpha}_{12}\left(1-\underline{\alpha}_{21} \underline{\alpha}_{21}\right)} \\
& \begin{cases}\leq 0, & \text { if } \bar{P}_{1} \geq \bar{P}_{2} \underline{\alpha}_{12} \\
\geq 0, & \text { if } \bar{P}_{1} \leq \bar{P}_{2} \underline{\alpha}_{12}\end{cases}
\end{aligned}
$$

where the inequalities are due to Assumption 2 that $l_{2} \geq \underline{\alpha}_{12} l_{1}$ and the fact that $0 \leq \underline{\alpha}_{21} \leq$ $1,0 \leq \underline{\alpha}_{12} \leq 1$.

From the above comparison as well as the results of Case 1-Case 3, we can draw the following conclusion on the best solution in subregions $\Omega_{1}, \Omega_{2}, \Omega_{3}$, and $\Omega_{4}$ :

(i) If $\bar{P}_{1} \leq \bar{P}_{2} \underline{\alpha}_{12}$, then we must have $\bar{P}_{2} \geq \bar{P}_{1} \underline{\alpha}_{21}$ since $\underline{\alpha}_{12}, \underline{\alpha}_{21} \in[0,1]$, and $\boldsymbol{t}_{3}^{3}$ dominates all the other points in subregions $\Omega_{1}, \Omega_{2}, \Omega_{3}$, and $\Omega_{4}$, since $f\left(\boldsymbol{t}_{3}^{3}\right) \geq f\left(\boldsymbol{t}_{2}^{6}\right) \geq f\left(\boldsymbol{t}_{4}^{7}\right), f\left(\boldsymbol{t}_{4}^{5}\right) \leq f\left(\boldsymbol{t}_{3}^{3}\right)$, and $f\left(\boldsymbol{t}_{4}^{3}\right) \leq f\left(\boldsymbol{t}_{3}^{3}\right)$.

(ii) If $\bar{P}_{1} \geq \bar{P}_{2} \underline{\alpha}_{12}$ and $\bar{P}_{2} \leq \bar{P}_{1} \underline{\alpha}_{21}$, then $\boldsymbol{t}_{4}^{3}$ or $\boldsymbol{t}_{4}^{5}$ dominates all the other points in subregions $\Omega_{1}, \Omega_{2}, \Omega_{3}$, and $\Omega_{4}$, since $f\left(\boldsymbol{t}_{4}^{5}\right) \geq f\left(\boldsymbol{t}_{4}^{7}\right) \geq f\left(\boldsymbol{t}_{2}^{5}\right)$, and $f\left(\boldsymbol{t}_{4}^{7}\right) \geq f\left(\boldsymbol{t}_{2}^{6}\right) \geq f\left(\boldsymbol{t}_{3}^{3}\right)$.

(iii) If $\bar{P}_{1} \geq \bar{P}_{2} \underline{\alpha}_{12}$ and $\bar{P}_{2} \geq \bar{P}_{1} \underline{\alpha}_{21}$, then $\boldsymbol{t}_{4}^{3}$ or $\boldsymbol{t}_{4}^{7}$ dominates all the other points in subregions $\Omega_{1}, \Omega_{2}, \Omega_{3}$, and $\Omega_{4}$, since $f\left(\boldsymbol{t}_{4}^{7}\right) \geq f\left(\boldsymbol{t}_{3}^{3}\right), f\left(\boldsymbol{t}_{4}^{7}\right) \geq f\left(\boldsymbol{t}_{2}^{6}\right) \geq f\left(\boldsymbol{t}_{3}^{3}\right)$, and $f\left(\boldsymbol{t}_{4}^{7}\right) \geq f\left(\boldsymbol{t}_{4}^{5}\right)$. 
Case 5. Suppose $\left(Q_{1}, Q_{2}\right) \in \Omega_{5}$, i.e., $D_{1}-l_{1} \leq Q_{1} \leq D_{1}, D_{2} \leq Q_{2}$.

In this case, $R(\boldsymbol{Q})=\max \left\{\bar{S}_{1}\left(Q_{1}-D_{1}+l_{1}\right)+\bar{S}_{2}\left(Q_{2}-D_{2}\right), \bar{S}_{2}\left(Q_{2}-D_{2}+l_{2}-\underline{\alpha}_{12}\left(D_{1}-Q_{1}\right)\right)\right\}$ and Model (33) becomes:

$$
\max _{\boldsymbol{Q}}\left\{f(\boldsymbol{Q})=\bar{P}_{1} Q_{1}+\bar{P}_{2} Q_{2}-R(\boldsymbol{Q}): D_{1}-l_{1} \leq Q_{1} \leq D_{1}, D_{2} \leq Q_{2}\right\}
$$

According to Observation 11, the optimal solution can be one of the following points: (1) the extreme points of $\Omega_{5}$, i.e, $\boldsymbol{t}_{5}^{1}, \boldsymbol{t}_{5}^{2}$; (2) the intersection point of line $\bar{S}_{1}\left(Q_{1}-D_{1}+l_{1}\right)+\bar{S}_{2}\left(Q_{2}-D_{2}\right)=$ $\bar{S}_{2}\left(Q_{2}-D_{2}+l_{2}-\underline{\alpha}_{12}\left(D_{1}-Q_{1}\right)\right)$ and the boundary of $\Omega_{5}$, i.e., $\boldsymbol{t}_{5}^{3}$ (See Figure 7 for an illustration). Note that $\boldsymbol{t}_{5}^{3} \in \Omega_{5}$ if $\bar{S}_{1} l_{1} \geq \bar{S}_{2} l_{2}$, otherwise, $\boldsymbol{t}_{5}^{3} \notin \Omega_{5}$. These possible solutions are listed in Table 7 .

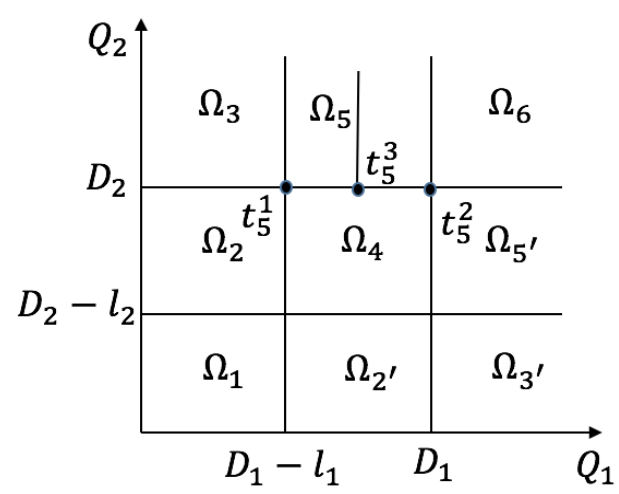

Figure 7: Possible solutions in Case 5

Table 7: Possible solutions and their total profits in Case 5

\begin{tabular}{|c|c|}
\hline Possible solutions & Total profit \\
\hline $\boldsymbol{t}_{5}^{1}=\boldsymbol{t}_{4}^{2}=\boldsymbol{t}_{3}^{2}=\boldsymbol{t}_{2}^{3}=\left(D_{1}-l_{1}, D_{2}\right)$ & $f\left(\boldsymbol{t}_{5}^{1}\right)=f\left(\boldsymbol{t}_{4}^{2}\right)=f\left(\boldsymbol{t}_{3}^{2}\right)=f\left(\boldsymbol{t}_{2}^{3}\right)=\bar{P}_{1}\left(D_{1}-l_{1}\right)+\bar{P}_{2} D_{2}-\bar{S}_{2}\left(l_{2}-\underline{\alpha}_{12} l_{1}\right)$ \\
\hline $\boldsymbol{t}_{5}^{2}=\boldsymbol{t}_{4}^{3}=\left(D_{1}, D_{2}\right)$ & $f\left(\boldsymbol{t}_{5}^{2}\right)=f\left(\boldsymbol{t}_{4}^{3}\right)=\bar{P}_{1} D_{1}+\bar{P}_{2} D_{2}-\max \left\{\bar{S}_{1} l_{1}, \bar{S}_{2} l_{2}\right\}$ \\
\hline $\boldsymbol{t}_{5}^{3}=\left(D_{1}-\frac{\bar{S}_{1} l_{1}-\bar{S}_{2} l_{2}}{\bar{S}_{1}-\bar{S}_{2} \underline{\underline{\alpha}}_{12}}, D_{2}\right)$ & $f\left(\boldsymbol{t}_{5}^{3}\right)=\bar{P}_{1} D_{1}+\bar{P}_{2} D_{2}-\bar{P}_{1} \frac{\bar{S}_{1} l_{1}-\bar{S}_{2} l_{2}}{\bar{S}_{1}-\bar{S}_{2} \underline{\alpha}_{12}}-\bar{S}_{1} \bar{S}_{2} \frac{l_{2}-l_{1} \underline{\alpha}_{12}}{\bar{S}_{1}-\bar{S}_{2} \underline{\alpha}_{12}}$ \\
\hline
\end{tabular}

In view of the results in Case 1- Case 4, the only new point is $\boldsymbol{t}_{5}^{3}$, which is in subregion 5 if $\bar{S}_{1} l_{1} \geq \bar{S}_{2} l_{2}$. Thus, suppose that $\bar{S}_{1} l_{1} \geq \bar{S}_{2} l_{2}$, we will compare $f\left(\boldsymbol{t}_{5}^{3}\right)$ with $f\left(\boldsymbol{t}_{3}^{3}\right), f\left(\boldsymbol{t}_{4}^{3}\right)$.

- Compare $f\left(\boldsymbol{t}_{4}^{3}\right)$ with $f\left(\boldsymbol{t}_{5}^{3}\right)$ :

$$
\begin{aligned}
f\left(\boldsymbol{t}_{4}^{3}\right)-f\left(\boldsymbol{t}_{5}^{3}\right) & =\bar{P}_{1} D_{1}+\bar{P}_{2} D_{2}-\bar{S}_{1} l_{1}-\bar{P}_{1} D_{1}-\bar{P}_{2} D_{2}+\bar{P}_{1} \frac{\bar{S}_{1} l_{1}-\bar{S}_{2} l_{2}}{\bar{S}_{1}-\bar{S}_{2} \underline{\alpha}_{12}}+\bar{S}_{1} \bar{S}_{2} \frac{l_{2}-l_{1} \underline{\alpha}_{12}}{\bar{S}_{1}-\bar{S}_{2} \underline{\alpha}_{12}} \\
& =-\frac{\left(\bar{S}_{1}-\bar{P}_{1}\right)\left(\bar{S}_{1} l_{1}-\bar{S}_{2} l_{2}\right)}{\bar{S}_{1}-\bar{S}_{2} \underline{\alpha}_{12}} \leq 0,
\end{aligned}
$$


where the inequality is due to $\bar{S}_{1} \geq \bar{P}_{1}$ and $\bar{S}_{1} l_{1} \geq \bar{S}_{2} l_{2}$.

- Compare $f\left(\boldsymbol{t}_{3}^{3}\right)$ with $f\left(\boldsymbol{t}_{5}^{3}\right)$

$$
\begin{aligned}
f\left(\boldsymbol{t}_{3}^{3}\right)-f\left(\boldsymbol{t}_{5}^{3}\right) & =\bar{P}_{2}\left(D_{2}-l_{2}+\underline{\alpha}_{12} D_{1}\right)-\bar{P}_{1} D_{1}-\bar{P}_{2} D_{2}+\bar{P}_{1} \frac{\bar{S}_{1} l_{1}-\bar{S}_{2} l_{2}}{\bar{S}_{1}-\bar{S}_{2} \underline{\alpha}_{12}}+\bar{S}_{1} \bar{S}_{2} \frac{l_{2}-l_{1} \underline{\alpha}_{12}}{\bar{S}_{1}-\bar{S}_{2} \underline{\alpha}_{12}} \\
& \geq \bar{P}_{2}\left(-l_{2}+\underline{\alpha}_{12} D_{1}\right)-\bar{P}_{1} D_{1}+\bar{P}_{1} \frac{\bar{S}_{1} l_{1}-\bar{S}_{2} l_{2}}{\bar{S}_{1}-\bar{S}_{2} \underline{\alpha}_{12}}+\bar{S}_{1} \bar{P}_{2} \frac{l_{2}-l_{1} \underline{\alpha}_{12}}{\bar{S}_{1}-\bar{S}_{2} \underline{\alpha}_{12}} \\
& =\left(\bar{P}_{2} \underline{\alpha}_{12}-\bar{P}_{1}\right)\left(D_{1}-\frac{\bar{S}_{1} l_{1}-\bar{S}_{2} l_{2}}{\bar{S}_{1}-\bar{S}_{2} \underline{\alpha}_{12}}\right) .
\end{aligned}
$$

Since $D_{1}-\frac{\bar{S}_{1} l_{1}-\bar{S}_{2} l_{2}}{\bar{S}_{1}-\bar{S}_{2} \underline{\alpha}_{12}} \geq D_{1}-l_{1} \geq 0$ and $\bar{S}_{1} l_{1} \geq \bar{S}_{2} l_{2}$, thus, $f\left(\boldsymbol{t}_{3}^{3}\right) \geq f\left(\boldsymbol{t}_{5}^{3}\right)$, if $\bar{P}_{1} \leq \bar{P}_{2} \underline{\alpha}_{12}$.

From the above comparison results as well as the results of Case 1-Case 4, we can draw the following conclusion on the best solution in subregions $\Omega_{1}, \Omega_{2}, \Omega_{3}, \Omega_{4}$, and $\Omega_{5}$ :

(i) If $\bar{P}_{1} \leq \bar{P}_{2} \underline{\alpha}_{12}$ and $\bar{P}_{2} \geq \bar{P}_{1} \underline{\alpha}_{21}$, then $\boldsymbol{t}_{3}^{3}$ dominates all the other points in subregions $\Omega_{1}, \Omega_{2}$, $\Omega_{3}, \Omega_{4}$, and $\Omega_{5}$, since $f\left(\boldsymbol{t}_{3}^{3}\right) \geq f\left(\boldsymbol{t}_{5}^{3}\right)$.

(ii) If $\bar{P}_{1} \geq \bar{P}_{2} \underline{\alpha}_{12}$ and $\bar{P}_{2} \leq \bar{P}_{1} \underline{\alpha}_{21}$, then

(a) if $\bar{S}_{1} l_{1} \leq \bar{S}_{2} l_{2}$, then $\boldsymbol{t}_{4}^{3}$ or $\boldsymbol{t}_{4}^{5}$ dominates all the other points in subregions $\Omega_{1}, \Omega_{2}, \Omega_{3}, \Omega_{4}$, and $\Omega_{5}$, and

(b) if $\bar{S}_{1} l_{1} \geq \bar{S}_{2} l_{2}$, then $\boldsymbol{t}_{5}^{3}$ or $\boldsymbol{t}_{4}^{5}$ dominates all the other points in subregions $\Omega_{1}, \Omega_{2}, \Omega_{3}, \Omega_{4}$, and $\Omega_{5}$.

(iii) If $\bar{P}_{1} \geq \bar{P}_{2} \underline{\alpha}_{12}$ and $\bar{P}_{2} \geq \bar{P}_{1} \underline{\alpha}_{21}$, then

(a) if $\bar{S}_{1} l_{1} \leq \bar{S}_{2} l_{2}$, then $\boldsymbol{t}_{4}^{3}$ or $\boldsymbol{t}_{4}^{7}$ dominates all the other points in subregions $\Omega_{1}, \Omega_{2}, \Omega_{3}, \Omega_{4}$, and $\Omega_{5}$, and

(b) if $\bar{S}_{1} l_{1} \geq \bar{S}_{2} l_{2}$, then $\boldsymbol{t}_{5}^{3}$ or $\boldsymbol{t}_{4}^{7}$ dominates all the other points in subregions $\Omega_{1}, \Omega_{2}, \Omega_{3}, \Omega_{4}$, and $\Omega_{5}$.

Case 6. Suppose $\left(Q_{1}, Q_{2}\right) \in \Omega_{6}$, i.e., $D_{1} \leq Q_{1}, D_{2} \leq Q_{2}$.

In this case, $R(\boldsymbol{Q})=\max \left\{\bar{S}_{1}\left(Q_{1}-D_{1}+l_{1}\right)+\bar{S}_{2}\left(Q_{2}-D_{2}\right), \bar{S}_{1}\left(Q_{1}-D_{1}\right)+\bar{S}_{2}\left(Q_{2}-D_{2}+l_{2}\right)\right\}$ and Model (33) becomes:

$$
\max _{\boldsymbol{Q}}\left\{f(\boldsymbol{Q})=\bar{P}_{1} Q_{1}+\bar{P}_{2} Q_{2}-R(\boldsymbol{Q}): D_{1} \leq Q_{1}, D_{2} \leq Q_{2}\right\}
$$

According to Observation 1, the optimal solution can only be $\boldsymbol{t}_{6}^{1}$, which is listed in Table 8 . 


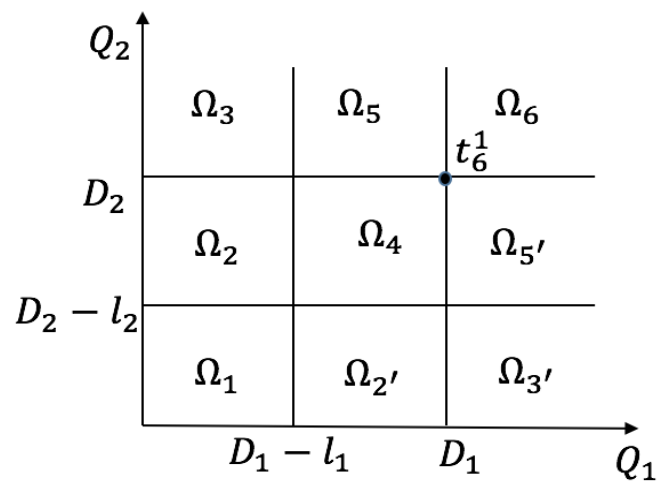

Figure 8: Possible solutions in Case 6

Table 8: Possible solutions and their total profits in Case 6

\begin{tabular}{|c|c|}
\hline Possible solutions & Total profit \\
\hline$t_{6}^{1}=t_{5}^{2}=t_{4}^{3}=\left(D_{1}, D_{2}\right)$ & $f\left(\boldsymbol{t}_{6}^{1}\right)=f\left(\boldsymbol{t}_{5}^{2}\right)=f\left(\boldsymbol{t}_{4}^{3}\right)=\bar{P}_{1} D_{1}+\bar{P}_{2} D_{2}-\max \left\{\bar{S}_{1} l_{1}, \bar{S}_{2} l_{2}\right\}$ \\
\hline
\end{tabular}

Note that there is no new optimal solution generated in the case; thus, the conclusion in Case 5 still follows.

Next, for the Cases $2^{\prime}, 3^{\prime}, 5^{\prime}$, since they are symmetric to Cases $2,3,5$, thus we will directly write down the possible solutions.

Case $2^{\prime}$. Suppose $\left(Q_{1}, Q_{2}\right) \in \Omega_{2^{\prime}}$, i.e., $D_{1} \leq Q_{1}, D_{2} \leq Q_{2}$.

Case $2^{\prime}$ is symmetric to Case 2 , and its possible solutions are listed in Table 9 .

Table 9: Possible solutions and their total profits in Case 2'

\begin{tabular}{|c|c|}
\hline Possible solutions & Total profit \\
\hline$t_{2^{\prime}}^{1}=\left(D_{1}-l_{1}, 0\right)$ & $f\left(\boldsymbol{t}_{2^{\prime}}^{1}\right)=\bar{P}_{1}\left(D_{1}-l_{1}\right)$ \\
\hline$t_{2^{\prime}}^{2}=\left(D_{1}, 0\right)$ & $f\left(\boldsymbol{t}_{2^{\prime}}^{2}\right)=\bar{P}_{1} D_{1}$ \\
\hline$t_{2^{\prime}}^{3}=\left(D_{1}, D_{2}-l_{2}\right)$ & $f\left(\boldsymbol{t}_{2^{\prime}}^{3}\right)=\bar{P}_{2}\left(D_{2}-l_{2}\right)+\bar{P}_{1} D_{1}-\bar{S}_{1}\left(l_{1}-\underline{\alpha}_{21} l_{2}\right)$ \\
\hline$t_{2^{\prime}}^{4}=t_{4}^{1}=t_{2}^{4}=t_{1}^{1}=\left(D_{1}-l_{1}, D_{2}-l_{2}\right)$ & $f\left(\boldsymbol{t}_{2^{\prime}}^{4}\right)=f\left(\boldsymbol{t}_{4}^{1}\right)=f\left(\boldsymbol{t}_{2}^{4}\right)=f\left(\boldsymbol{t}_{1}^{1}\right)=\bar{P}_{2}\left(D_{2}-l_{2}\right)+\bar{P}_{1}\left(D_{1}-l_{1}\right)$ \\
\hline$t_{2^{\prime}}^{5}=\left(D_{1}, D_{2}-l_{1} / \underline{\alpha}_{21}\right)$ & $f\left(\boldsymbol{t}_{2^{\prime}}^{5}\right)=\bar{P}_{2}\left(D_{2}-l_{1} / \underline{\alpha}_{21}\right)+\bar{P}_{1} D_{1}$ \\
\hline$t_{2^{\prime}}^{6}=\left(D_{1}-l_{1}+\underline{\alpha}_{21} l_{2}, D_{2}-l_{2}\right)$ & $f\left(\boldsymbol{t}_{2^{\prime}}^{6}\right)=\bar{P}_{2}\left(D_{2}-l_{2}\right)+\bar{P}_{1}\left(D_{1}-l_{1}+\underline{\alpha}_{21} l_{2}\right)$ \\
\hline
\end{tabular}

Case $3^{\prime}$. Suppose $\left(Q_{1}, Q_{2}\right) \in \Omega_{3^{\prime}}$, i.e., $D_{1} \leq Q_{1}, 0 \leq Q_{2} \leq D_{2}-l_{2}$.

Case $3^{\prime}$ is symmetric to Case 3 and its possible solutions are listed in Table 10 . 
Table 10: Possible solutions and their total profits in Case 3'

\begin{tabular}{|c|c|}
\hline Possible solutions & Total profit \\
\hline$t_{3^{\prime}}^{1}=t_{2^{\prime}}^{2}=\left(D_{1}, 0\right)$ & $f\left(\boldsymbol{t}_{3^{\prime}}^{1}\right)=f\left(\boldsymbol{t}_{2^{\prime}}^{2}\right)=\bar{P}_{1} D_{1}$ \\
\hline$t_{3^{\prime}}^{2}=t_{2^{\prime}}^{3}=\left(D_{1}, D_{2}-l_{2}\right)$ & $f\left(\boldsymbol{t}_{3^{\prime}}^{2}\right)=f\left(\boldsymbol{t}_{2^{\prime}}^{3}\right)=\bar{P}_{2}\left(D_{2}-l_{2}\right)+\bar{P}_{1} D_{1}-\bar{S}_{1}\left(l_{1}-\underline{\alpha}_{21} l_{2}\right)$ \\
\hline$t_{3^{\prime}}^{3}=\left(D_{1}-l_{1}+\underline{\alpha}_{21} D_{2}, 0\right)$ & $f\left(\boldsymbol{t}_{3^{\prime}}^{3}\right)=\bar{P}_{1}\left(D_{1}-l_{1}+\underline{\alpha}_{21} D_{2}\right)$ \\
\hline$t_{3^{\prime}}^{4}=t_{2^{\prime}}^{5}=\left(D_{1}, D_{2}-l_{1} / \underline{\alpha}_{21}\right)$ & $f\left(\boldsymbol{t}_{3^{\prime}}^{4}\right)=f\left(\boldsymbol{t}_{2^{\prime}}^{5}\right)=\bar{P}_{2}\left(D_{2}-l_{1} / \underline{\alpha}_{21}\right)+\bar{P}_{1} D_{1}$ \\
\hline
\end{tabular}

Case $5^{\prime}$. Suppose $\left(Q_{1}, Q_{2}\right) \in \Omega_{5^{\prime}}$, i.e., $D_{1} \leq Q_{1}, D_{2}-l_{2} \leq Q_{2} \leq D_{2}$.

Case $5^{\prime}$ is symmetric to Case 5 and its possible solutions are listed in Table 11 .

Table 11: Possible solutions and their total profits for Case 5'

\begin{tabular}{|c|c|}
\hline Possible solutions & Total profit \\
\hline$t_{5^{\prime}}^{1}=t_{4}^{2}=t_{3^{\prime}}^{2}=t_{2^{\prime}}^{3}=\left(D_{1}, D_{2}-l_{2}\right)$ & $f\left(\boldsymbol{t}_{5^{\prime}}^{1}\right)=f\left(\boldsymbol{t}_{4}^{2}\right)=f\left(\boldsymbol{t}_{3^{\prime}}^{2}\right)=f\left(\boldsymbol{t}_{2^{\prime}}^{3}\right)=\bar{P}_{2}\left(D_{2}-l_{2}\right)+\bar{P}_{1} D_{1}-\bar{S}_{1}\left(l_{1}-\underline{\alpha}_{21} l_{2}\right)$ \\
\hline$t_{5^{\prime}}^{2}=t_{5}^{2}=t_{4}^{1}=\left(D_{1}, D_{2}\right)$ & $f\left(\boldsymbol{t}_{5^{\prime}}^{2}\right)=f\left(\boldsymbol{t}_{5}^{2}\right)=f\left(\boldsymbol{t}_{4}^{1}\right)=\bar{P}_{2} D_{2}+\bar{P}_{1} D_{1}-\max \left\{\bar{S}_{2} l_{2}, \bar{S}_{1} l_{1}\right\}$ \\
\hline$t_{5^{\prime}}^{3}=\left(D_{1}, D_{2}-\frac{\bar{S}_{2} l_{2}-\bar{S}_{1} l_{1}}{\bar{S}_{2}-\bar{S}_{1} \underline{\alpha}_{21}}\right)$ & $f\left(\boldsymbol{t}_{5^{\prime}}^{3}\right)=\bar{P}_{1} D_{1}+\bar{P}_{2} D_{2}-\bar{P}_{2}{\overline{S_{2}}}_{l_{2}-\bar{S}_{1} \bar{S}_{1} \underline{\alpha}_{1}}-\bar{S}_{1} \bar{S}_{2} \frac{l_{1}-l_{2} \underline{\alpha}_{21}}{\bar{S}_{2}-\bar{S}_{1} \underline{\alpha}_{21}}$ \\
\hline
\end{tabular}

Based on the results in Case 1-Case 6, thus symmetricly, we can also draw the following conclusions in the subregions $\Omega_{1}, \Omega_{2^{\prime}}, \Omega_{3^{\prime}}, \Omega_{4}, \Omega_{5^{\prime}}$ and $\Omega_{6}$ :

(i) If $\bar{P}_{1} \leq \bar{P}_{2} \underline{\alpha}_{12}$ and $\bar{P}_{2} \geq \bar{P}_{1} \underline{\alpha}_{21}$, then

(a) if $\bar{S}_{1} l_{1} \geq \bar{S}_{2} l_{2}$, then $\boldsymbol{t}_{4}^{3}$ or $\boldsymbol{t}_{4}^{5}$ dominates all the other points in subregions $\Omega_{1}, \Omega_{2^{\prime}}, \Omega_{3^{\prime}}, \Omega_{4}$, and $\Omega_{5^{\prime}}$.

(b) if $\bar{S}_{1} l_{1} \leq \bar{S}_{2} l_{2}$, then $\boldsymbol{t}_{5^{\prime}}^{3}$ or $\boldsymbol{t}_{4}^{5}$ dominates all the other points in subregions $\Omega_{1}, \Omega_{2^{\prime}}, \Omega_{3^{\prime}}$, $\Omega_{4}$, and $\Omega_{5^{\prime}}$, since $f\left(\boldsymbol{t}_{4}^{3}\right) \leq f\left(\boldsymbol{t}_{5^{\prime}}^{3}\right)$.

(ii) If $\bar{P}_{1} \geq \bar{P}_{2} \underline{\alpha}_{12}$ and $\bar{P}_{2} \leq \bar{P}_{1} \underline{\alpha}_{21}$, then $\boldsymbol{t}_{3^{\prime}}^{3}$ dominates all the other points in subregions $\Omega_{1}$, $\Omega_{2^{\prime}}, \Omega_{3^{\prime}}, \Omega_{4}$, and $\Omega_{5^{\prime}}$.

(iii) If $\bar{P}_{1} \geq \bar{P}_{2} \underline{\alpha}_{12}$ and $\bar{P}_{2} \geq \bar{P}_{1} \underline{\alpha}_{21}$, then

(a) if $\bar{S}_{1} l_{1} \geq \bar{S}_{2} l_{2}$, then $\boldsymbol{t}_{4}^{3}$ or $\boldsymbol{t}_{4}^{7}$ dominates all the other points in subregions $\Omega_{1}, \Omega_{2^{\prime}}, \Omega_{3^{\prime}}, \Omega_{4}$, and $\Omega_{5^{\prime}}$, and

(b) if $\bar{S}_{1} l_{1} \leq \bar{S}_{2} l_{2}$, then $\boldsymbol{t}_{5^{\prime}}^{3}$ or $\boldsymbol{t}_{4}^{7}$ dominates all the other points in subregions $\Omega_{1}, \Omega_{2^{\prime}}, \Omega_{3^{\prime}}$, $\Omega_{4}$, and $\Omega_{5^{\prime}}$, since $f\left(\boldsymbol{t}_{4}^{3}\right) \leq f\left(\boldsymbol{t}_{5^{\prime}}^{3}\right)$. 
Thus, combining all the comparison results, we can conclude that

Case 1: If $\bar{P}_{1} \leq \bar{P}_{2} \underline{\alpha}_{12}$ and $\bar{P}_{2} \geq \bar{P}_{1} \underline{\alpha}_{21}$, then $\boldsymbol{t}_{3}^{3}$ dominates all the other points of the 9 subregions, since $f\left(\boldsymbol{t}_{4}^{5}\right) \leq f\left(\boldsymbol{t}_{3}^{3}\right), f\left(\boldsymbol{t}_{4}^{3}\right) \leq f\left(\boldsymbol{t}_{3}^{3}\right)$ from the results of Case 4 , and when $\bar{S}_{1} l_{1} \leq \bar{S}_{2} l_{2}$, we have

$$
\begin{aligned}
f\left(\boldsymbol{t}_{5^{\prime}}^{3}\right)-f\left(\boldsymbol{t}_{3}^{3}\right) & =\bar{P}_{1} D_{1}+\bar{P}_{2} D_{2}-\bar{P}_{2} \frac{\bar{S}_{2} l_{2}-\bar{S}_{1} l_{1}}{\bar{S}_{2}-\bar{S}_{1} \underline{\alpha}_{21}}-\bar{S}_{1} \bar{S}_{2} \frac{l_{1}-l_{2} \underline{\alpha}_{21}}{\bar{S}_{2}-\bar{S}_{1} \underline{\alpha}_{21}}-\left(\bar{P}_{2}\left(D_{2}-l_{2}+\underline{\alpha}_{12} D_{1}\right)\right) \\
& =\left(\bar{P}_{1}-\bar{P}_{2} \underline{\alpha}_{12}\right) D_{1}+\bar{S}_{1}\left(\bar{S}_{2}-\bar{P}_{2}\right) \frac{l_{2} \underline{\alpha}_{21}-l_{1}}{\bar{S}_{2}-\bar{S}_{1} \underline{\alpha}_{21}} \leq 0
\end{aligned}
$$

where the inequality is due to $\bar{P}_{1} \leq \bar{P}_{2} \underline{\alpha}_{12}, \bar{S}_{1} \geq 0, \bar{S}_{2}-\bar{P}_{2} \geq 0, l_{2} \underline{\alpha}_{21} \leq l_{1}$ and $\bar{S}_{2} \geq \bar{S}_{1} \underline{\alpha}_{21}$ (due to $\bar{S}_{1} l_{1} \leq \bar{S}_{2} l_{2}$ and $l_{2} \underline{\alpha}_{21} \leq l_{1}$ ).

Case 2: If $\bar{P}_{2} \leq \bar{P}_{1} \underline{\alpha}_{21}$ and $\bar{P}_{1} \geq \bar{P}_{2} \underline{\alpha}_{12}$, then $\boldsymbol{t}_{3^{\prime}}^{3}$ dominates all the other points of the 9 subregions, since $f\left(\boldsymbol{t}_{4}^{5}\right) \leq f\left(\boldsymbol{t}_{3^{\prime}}^{3}\right), f\left(\boldsymbol{t}_{4}^{3}\right) \leq f\left(\boldsymbol{t}_{3^{\prime}}^{3}\right)$ which is due to the symmetric results from Case 4 that $f\left(\boldsymbol{t}_{4}^{5}\right) \leq f\left(\boldsymbol{t}_{3}^{3}\right), f\left(\boldsymbol{t}_{4}^{3}\right) \leq f\left(\boldsymbol{t}_{3}^{3}\right)$, and when $\bar{S}_{1} l_{1} \geq \bar{S}_{2} l_{2}$, by the results that $f\left(\boldsymbol{t}_{5^{\prime}}^{3}\right) \leq f\left(\boldsymbol{t}_{3}^{3}\right)$, we must have $f\left(\boldsymbol{t}_{5}^{3}\right) \leq f\left(\boldsymbol{t}_{3^{\prime}}^{3}\right)$.

Case 3: If $\bar{P}_{1} \geq \bar{P}_{2} \underline{\alpha}_{12}, \bar{P}_{2} \geq \bar{P}_{1} \underline{\alpha}_{21}$, there we can separate the results into two sub-cases:

Sub-case 3.1: If $\bar{S}_{1} l_{1} \geq \bar{S}_{2} l_{2}, \boldsymbol{t}_{5}^{3}$ or $\boldsymbol{t}_{4}^{7}$ dominates all the other points, since $f\left(\boldsymbol{t}_{5}^{3}\right) \geq f\left(\boldsymbol{t}_{4}^{3}\right)$ from Case 5;

Sub-case 3.2: If $\bar{S}_{1} l_{1} \leq \bar{S}_{2} l_{2}, \boldsymbol{t}_{5^{\prime}}^{3}$ or $\boldsymbol{t}_{4}^{7}$ dominates all the other points, since $f\left(\boldsymbol{t}_{5^{\prime}}^{3}\right) \geq f\left(\boldsymbol{t}_{4}^{3}\right)$ by symmetry.

This completes the proof.

\section{A.4 Proof of Theorem 3}

Theorem 3. When $\underline{\boldsymbol{\alpha}}=0$, the optimal solutions $\boldsymbol{Q}^{*}$ of Model (16) are characterized as follows:

(i) If $\sum_{i \in[n]} \bar{P}_{i} \leq k$, then $Q_{i}^{*}=D_{i}-l_{i}$, and $v^{*}=\sum_{i \in[n]} \bar{P}_{i}\left(D_{i}-l_{i}\right)$.

(ii) If $\sum_{i \in[n]} \frac{\bar{P}_{i}}{\bar{S}_{i}}>k$,

$$
Q_{i}^{*}= \begin{cases}D_{i}-l_{i}+\frac{\bar{S}_{(t+1)} l_{(t+1)},}{\bar{S}_{i}}, & \text { if } i \in T \\ D_{i}, & \text { if } i \in[n] \backslash T\end{cases}
$$

and

$$
v^{*}=\sum_{i \in[n] \backslash T} \bar{P}_{i} l_{i}-\bar{S}_{(t+1)} l_{(t+1)} k+\sum_{i \in T} \frac{\bar{P}_{i}}{\bar{S}_{i}} \bar{S}_{(t+1)} l_{(t+1)}+\sum_{i \in[n]} \bar{P}_{i}\left(D_{i}-l_{i}\right),
$$

where set $T:=\{(1),(2), \cdots,(t)\}$ satisfying $\sum_{i \in T} \frac{\bar{P}_{i}}{\bar{S}_{i}} \leq k, \sum_{i \in T \cup\{(t+1)\}} \frac{\bar{P}_{i}}{\bar{S}_{i}}>k$. 
Proof. Let $\widehat{X}=\left\{\boldsymbol{z}: \sum_{i \in[n]} z_{i} \leq k, z_{i} \in[0,1], \forall i \in[n]\right\}$, which is a well-known integral polytope. Thus, $\operatorname{conv}(X)=\widehat{X}$ and the inner maximization problem of $(15)$ is equivalent to maximize a linear function of set $\widehat{X}$. Thus, we have

$$
v^{*}=\max _{\boldsymbol{Q} \in[\boldsymbol{D}-l, \boldsymbol{D}]}\left(\sum_{i \in[n]} \bar{P}_{i} Q_{i}-\max _{\boldsymbol{z} \in \widehat{X}} \sum_{i \in[n]} \bar{S}_{i}\left(Q_{i}-D_{i}+l_{i}\right) z_{i}\right) .
$$

Let $q_{i}=Q_{i}-D_{i}+l_{i}$ for each $i \in[n]$, then Model (34) is equivalent to

$$
v^{*}=\max _{\boldsymbol{q} \in[\mathbf{0}, \boldsymbol{l}]} \min _{\boldsymbol{z} \in \widehat{X}}\left(\sum_{i \in[n]}\left(\bar{P}_{i}-\bar{S}_{i} z_{i}\right) q_{i}+\sum_{i \in[n]} \bar{P}_{i}\left(D_{i}-l_{i}\right)\right) .
$$

Let $\lambda$ be the dual variable associated with constraint $\sum_{i \in[n]} z_{i} \leq k$ and $\beta_{i}$ be the dual variable associated with constraint $z_{i} \leq 1$ for each $i \in[n]$. Then by reformulating the inner maximization into its dual form, Model (35) is equivalent to

$$
\begin{aligned}
& v^{*}=\max _{\boldsymbol{q}, \lambda, \boldsymbol{\beta}} k \lambda+\sum_{i \in[n]} \beta_{i}+\sum_{i \in[n]} \bar{P}_{i} q_{i}+\sum_{i \in[n]} \bar{P}_{i}\left(D_{i}-l_{i}\right) \\
& \text { s.t. } \lambda+\beta_{i} \leq-\bar{S}_{i} q_{i}, i \in[n] \text {, } \\
& 0 \leq q_{i} \leq l_{i}, i \in[n], \\
& \lambda, \beta_{i} \leq 0, i \in[n] .
\end{aligned}
$$

In Model (35), since the objective function is concave in $\boldsymbol{q}$ and convex in $\boldsymbol{z}$, and set $\widehat{X}$ is convex compact set, thus according to Sion's minimax theorem (Sion, 1958), we can equivalently reformulate Model (35) by switching the min with max operators as follows:

$$
v^{*}=\min _{\boldsymbol{z} \in \widehat{X}} \max _{\boldsymbol{q} \in[\mathbf{0}, \boldsymbol{l}]}\left\{\sum_{i \in[n]}\left(\bar{P}_{i}-\bar{S}_{i} z_{i}\right) q_{i}+\sum_{i \in[n]} \bar{P}_{i}\left(D_{i}-l_{i}\right)\right\} .
$$

Note that $\max _{q_{i} \in\left[0, l_{i}\right]}\left(\sum_{i \in[n]}\left(\bar{P}_{i}-\bar{S}_{i} z_{i}\right) q_{i}\right)=\sum_{i \in[n]}\left(\bar{P}_{i}-\bar{S}_{i} z_{i}\right)_{+} l_{i}$ for each $i \in[n]$. Thus, Model (35) is equivalent to

$$
v^{*}=\min _{\boldsymbol{z} \in \hat{X}}\left\{\sum_{i \in[n]}\left(\bar{P}_{i}-\bar{S}_{i} z_{i}\right)_{+} l_{i}+\sum_{i \in[n]} \bar{P}_{i}\left(D_{i}-l_{i}\right)\right\} .
$$

we also observe that 
Claim 2. Model (37) is equivalent to

$$
v^{*}=\min _{\boldsymbol{z} \in \widehat{X}_{1}}\left\{\sum_{i \in[n]}\left(\bar{P}_{i}-\bar{S}_{i} z_{i}\right) l_{i}+\sum_{i \in[n]} \bar{P}_{i}\left(D_{i}-l_{i}\right)\right\},
$$

where $\widehat{X}_{1}=\left\{z: \sum_{i \in[n]} z_{i} \leq k, 0 \leq z_{i} \leq \frac{\bar{P}_{i}}{\bar{S}_{i}}\right\}$.

Proof. Let $v_{1}^{*}$ denote the optimal value of Model (38). To prove Model (37) is equivalent to Model (38), we only need to show $v^{*}=v_{1}^{*}$.

$v^{*} \geq v_{1}^{*}$. Given an optimal solution $\boldsymbol{z}^{*}$ of Model (37), let us define set $\mathcal{J}_{1}=\left\{i \in[n]: 0 \leq z_{i}^{*} \leq \frac{\bar{P}_{i}}{\bar{S}_{i}}\right\}$ and $\mathcal{J}_{2}=\left\{i \in[n]: \frac{\bar{P}_{i}}{\bar{S}_{i}}<z_{i}^{*} \leq 1\right\}$. Clearly, $\mathcal{J}_{1} \cup \mathcal{J}_{2}=[n]$ and $\mathcal{J}_{1} \cap \mathcal{J}_{2}=\emptyset$. Next, we define a new solution $\widehat{z}$ such that

$$
\widehat{z_{i}}=\left\{\begin{array}{ll}
z_{i}^{*}, & \text { if } i \in \mathcal{J}_{1} \\
\bar{P}_{i} & \text { otherwise }
\end{array},\right.
$$

for each $i \in[n]$. Clearly, $\widehat{\boldsymbol{z}} \in \widehat{X}_{1}$. We also have

$$
\begin{aligned}
v^{*} & =\sum_{i \in[n]}\left(\bar{P}_{i}-\bar{S}_{i} z_{i}^{*}\right)_{+} l_{i}+\sum_{i \in[n]} \bar{P}_{i}\left(D_{i}-l_{i}\right) \\
& =\sum_{i \in \mathcal{J}_{1}}\left(\bar{P}_{i}-\bar{S}_{i} z_{i}^{*}\right) l_{i}+\sum_{i \in[n]} \bar{P}_{i}\left(D_{i}-l_{i}\right) \\
& =\sum_{i \in[n]}\left(\bar{P}_{i}-\bar{S}_{i} \widehat{z}_{i}\right) l_{i}+\sum_{i \in[n]} \bar{P}_{i}\left(D_{i}-l_{i}\right),
\end{aligned}
$$

where the third equality is due to the definition of $\widehat{\boldsymbol{z}}$. Therefore, $\widehat{\boldsymbol{z}}$ is feasible to Model (38) with the same objective value $v^{*}$. Thus, we have $v^{*} \geq v_{1}^{*}$.

$v^{*} \leq v_{1}^{*}$. Since $\widehat{X}_{1} \subseteq \widehat{X}$, thus, $v^{*} \leq v_{1}^{*}$.

Note that Model (38) is a continuous knapsack minimization problem and can be solved by greedy procedure (c.f., Dantzig (1957); Levi et al. (2014)). Let $\boldsymbol{z}^{*}$ denote an optimal solution to Model (38). To obtain $\boldsymbol{z}^{*}$, we first sort $\left\{\bar{S}_{i} l_{i}\right\}_{i \in[n]}$ in the descending order $\bar{S}_{(1)} l_{(1)} \geq \bar{S}_{(2)} l_{(2)} \geq$ $\cdots \geq \bar{S}_{(n)} l_{(n)}$. Next, we discuss two cases:

Case 1. If $\sum_{i \in[n]} \frac{\bar{P}_{i}}{\bar{S}_{i}} \leq k$, then we have

$$
z_{i}^{*}=\frac{\bar{P}_{i}}{\bar{S}_{i}}, \forall i \in[n]
$$

and $v^{*}=\sum_{i \in[n]} \bar{P}_{i}\left(D_{i}-l_{i}\right)$. On the other hand, in $(36)$, let us consider the following feasible solution $\lambda^{*}=0, \beta_{i}^{*}=0, q_{i}^{*}=0$ for all $i \in[n]$ with objective value equal to 
$\sum_{i \in[n]} \bar{P}_{i}\left(D_{i}-l_{i}\right)$. Therefore, $\left(\boldsymbol{Q}^{*}, \lambda^{*}, \boldsymbol{\beta}^{*}\right)$ is optimal to 36 . Hence, the optimal order quantity for each product $i \in[n]$ is

$$
Q_{i}^{*}=q_{i}^{*}+D_{i}-l_{i}=D_{i}-l_{i}
$$

Case 2. If $\sum_{i} \frac{\bar{P}_{i}}{\bar{S}_{i}}>k$, then let us define set $T:=\{(1),(2), \ldots,(t)\}$ such that $\sum_{i \in T} \frac{\bar{P}_{i}}{\bar{S}_{i}} \leq k$, $\sum_{i \in T \cup(t+1)} \frac{\bar{P}_{i}}{\bar{S}_{i}}>k$. Then we have

$$
z_{i}^{*}= \begin{cases}\frac{\bar{P}_{i}}{\bar{S}_{i}} & \text { if } i \in T \\ k-\sum_{\tau \in T} \frac{\bar{P}_{\tau}}{\bar{S}_{\tau}} & \text { if } i=(t+1), \\ 0 & \text { otherwise }\end{cases}
$$

for each $i \in[n]$, and

$$
\begin{aligned}
v^{*} & =\sum_{i \in[n] \backslash T}\left(\bar{P}_{i}-\bar{S}_{i} z_{i}^{*}\right) l_{i}+\sum_{i \in[n]} \bar{P}_{i}\left(D_{i}-l_{i}\right) \\
& =\bar{P}_{(t+1)} l_{(t+1)}-\bar{S}_{(t+1)}\left(k-\sum_{i \in T} \frac{\bar{P}_{i}}{\bar{S}_{i}}\right) l_{(t+1)}+\sum_{i \in\{[n] \backslash T \cup(t+1)\}} \bar{P}_{i} l_{i}+\sum_{i \in[n]} \bar{P}_{i}\left(D_{i}-l_{i}\right) \\
& =\sum_{i \in[n] \backslash T} \bar{P}_{i} l_{i}-\bar{S}_{(t+1)} l_{(t+1)} k+\sum_{i \in T} \bar{P}_{i} \bar{S}_{i} \bar{S}_{(t+1)} l_{(t+1)}+\sum_{i \in[n]} \bar{P}_{i}\left(D_{i}-l_{i}\right)
\end{aligned}
$$

Next $\lambda^{*}=-\bar{S}_{t+1} l_{t+1}, \beta_{i}^{*}=0$ and

$$
q_{i}^{*}= \begin{cases}\frac{\bar{S}_{t+1} l_{t+1}}{\bar{S}_{i}}, & \text { if } i \in T \\ l_{i}, & \text { if } i \in[n] \backslash T\end{cases}
$$

for each product $i \in[n]$. Clearly, $\left(\boldsymbol{Q}^{*}, \lambda^{*}, \boldsymbol{\beta}^{*}\right)$ is feasible to (36) with objective value equal to $v^{*}$. Therefore, $\left(\boldsymbol{Q}^{*}, \lambda^{*}, \boldsymbol{\beta}^{*}\right)$ is optimal to $(36)$. Hence, the optimal order quantity for each product $i \in[n]$ is

$$
Q_{i}^{*}=q_{i}^{*}+D_{i}-l_{i}=\left\{\begin{array}{ll}
D_{i}-l_{i}+\frac{\bar{S}_{t+1} l_{t+1}}{\bar{S}_{i}}, & \text { if } i \in T \\
D_{i}, & \text { if } i \in[n] \backslash T
\end{array} .\right.
$$




\section{A.5 Proof of Proposition 3}

Proposition 3. The inner maximization problem (20) is equivalent to

$$
\begin{aligned}
R(\boldsymbol{Q}, \boldsymbol{u}, \boldsymbol{\psi})=\max _{\boldsymbol{x}, \boldsymbol{y}, \boldsymbol{z}} \sum_{i \in[n]} \bar{S}_{i}\left[\left(Q_{i}-D_{i}\right) x_{i}+l_{i} y_{i i}-\sum_{j \in[n]} \underline{\alpha}_{j i}\left(u_{j} y_{j i}+\psi_{j}\left(x_{i}-y_{j i}\right)\right)\right] \\
\text { s.t. } \sum_{i \in[n]} z_{i} \leq k . \\
\quad y_{j i} \leq x_{i}, \forall i, j \in[n], \\
\quad y_{j i} \leq z_{j}, \forall i, j \in[n], \\
\quad z_{i}, x_{i} \in\{0,1\}, y_{j i} \geq 0, \forall i, j \in[n] .
\end{aligned}
$$

Proof. Let $\widehat{R}(\boldsymbol{Q}, \boldsymbol{u}, \boldsymbol{\psi})$ denote the optimal value of Model $(22)$. It is sufficient to show that $R(\boldsymbol{Q}, \boldsymbol{u}, \boldsymbol{\psi})=\widehat{R}(\boldsymbol{Q}, \boldsymbol{u}, \boldsymbol{\psi})$ for any feasible $(\boldsymbol{Q}, \boldsymbol{u}, \boldsymbol{\psi}) \in \mathbb{R}_{+}^{3 n}$.

$R(\boldsymbol{Q}, \boldsymbol{u}, \boldsymbol{\psi}) \leq \widehat{R}(\boldsymbol{Q}, \boldsymbol{u}, \boldsymbol{\psi})$. Suppose $\left(\boldsymbol{x}^{*}, \boldsymbol{z}^{*}\right) \in\{0,1\}^{2 n}$ is an optimal solution of Model (21). Define $y_{j i}^{*}=z_{j}^{*} x_{i}^{*}$ for each $i, j \in[n]$. Clearly, $\left(\boldsymbol{x}^{*}, \boldsymbol{y}^{*}, \boldsymbol{z}^{*}\right)$ is feasible to Model 222) and

$$
\begin{aligned}
R(\boldsymbol{Q}, \boldsymbol{u}, \boldsymbol{\psi}) & =\sum_{i \in[n]} \bar{S}_{i}\left[Q_{i}-D_{i}+l_{i} z_{i}^{*}-\sum_{j} \underline{\alpha}_{j i}\left(u_{j} z_{j}^{*}+v_{j}\left(1-z_{j}^{*}\right)\right)\right] x_{i}^{*} \\
& =\sum_{i \in[n]} \bar{S}_{i}\left[\left(Q_{i}-D_{i}\right) x_{i}^{*}+l_{i} y_{i i}^{*}-\sum_{j \in[n]} \underline{\alpha}_{j i}\left(u_{j} y_{j i}^{*}+\psi_{j}\left(x_{i}^{*}-y_{j i}^{*}\right)\right)\right],
\end{aligned}
$$

i.e., it yields the same objective value as $R(\boldsymbol{Q}, \boldsymbol{u}, \boldsymbol{\psi})$. Thus, $R(\boldsymbol{Q}, \boldsymbol{u}, \boldsymbol{\psi}) \leq \widehat{R}(\boldsymbol{Q}, \boldsymbol{u}, \boldsymbol{\psi})$.

$R(\boldsymbol{Q}, \boldsymbol{u}, \boldsymbol{\psi}) \geq \widehat{R}(\boldsymbol{Q}, \boldsymbol{u}, \boldsymbol{\psi})$. Suppose $\left(\boldsymbol{x}^{*}, \boldsymbol{y}^{*}, \boldsymbol{z}^{*}\right)$ is an optimal solution to Model 22 . Since both $\boldsymbol{x}^{*}$ and $\boldsymbol{z}^{*}$ are binary, thus according to constraints $22 \mathrm{c}$ ) and $22 \mathrm{~d}$ ), we must have $y_{j i}^{*} \leq$ $z_{j}^{*} x_{i}^{*}=\min \left\{z_{j}^{*}, x_{i}^{*}\right\}$ for each $i, j \in[n]$. Therefore,

$$
\begin{aligned}
\widehat{R}(\boldsymbol{Q}, \boldsymbol{u}, \boldsymbol{\psi}) & =\sum_{i \in[n]} \bar{S}_{i}\left[\left(Q_{i}-D_{i}\right) x_{i}^{*}+l_{i} y_{i i}^{*}-\sum_{j \in[n]} \underline{\alpha}_{j i}\left(u_{j} y_{j i}^{*}+\psi_{j}\left(x_{i}^{*}-y_{j i}^{*}\right)\right)\right] \\
& \leq \sum_{i \in[n]} \bar{S}_{i}\left[Q_{i}-D_{i}+l_{i} z_{i}^{*}-\sum_{j} \underline{\alpha}_{j i}\left(u_{j} z_{j}^{*}+v_{j}\left(1-z_{j}^{*}\right)\right)\right] x_{i}^{*}
\end{aligned}
$$

where the first inequality is due to the coefficients of $\left\{y_{j i}^{*}\right\}_{j, i \in[n]}$ are all nonnegative, i.e., $\bar{S}_{i} l_{i} \geq 0$ and $\psi_{j} \geq u_{j}$ for all $i, j \in[n]$. Hence, $\left(\boldsymbol{x}^{*}, \boldsymbol{z}^{*}\right)$ is feasible to Model (21) and yields an objective value at least as large as $\widehat{R}(\boldsymbol{Q}, \boldsymbol{u}, \boldsymbol{\psi})$, which implies that $R(\boldsymbol{Q}, \boldsymbol{u}, \boldsymbol{\psi}) \geq \widehat{R}(\boldsymbol{Q}, \boldsymbol{u}, \boldsymbol{\psi})$. 


\section{A.6 Proof of Theorem 5}

Theorem 5. Let $v^{C A}$ denote the optimal value of Model (28). Then

(i) $v^{C A} \leq v^{*} ;$ and

(ii) $v^{C A}=v^{*}$, if one of the following conditions holds: (1) $\underline{\boldsymbol{\alpha}}=\mathbf{0}$, or (2) $n=k$.

Proof. $v^{*} \geq v^{C A}$ holds since in (28), we replace set $\Xi$ to be its continuous relaxation $\Xi_{C}$. It remains to show that $v^{*}=v^{C A}$ if $\underline{\boldsymbol{\alpha}}=\mathbf{0}$ or $n=k$. To prove this result, it is sufficient to show that for any given $(\boldsymbol{Q}, \boldsymbol{u}, \boldsymbol{\psi})$ satisfying constraints $23 \mathrm{a}-22 \mathrm{e}, 24 \mathrm{~b}-24 \mathrm{c}$, the following linear program

$$
\max _{(\boldsymbol{x}, \boldsymbol{y}, \boldsymbol{z}) \in \Xi_{C}} g(\boldsymbol{Q}, \boldsymbol{u}, \boldsymbol{\psi}, \boldsymbol{x}, \boldsymbol{y}, \boldsymbol{z})
$$

has an integral optimal solution, i.e., the continuous relaxation of Model 22 has an integral optimal solution.

$\underline{\boldsymbol{\alpha}}=\mathbf{0}$. In this case, Model 22 is equivalent to

$$
\begin{aligned}
R(\boldsymbol{Q}, \boldsymbol{u}, \boldsymbol{\psi})=\max _{\boldsymbol{x}, \boldsymbol{y}, \boldsymbol{z}} & \sum_{i \in[n]} \bar{S}_{i}\left[\left(Q_{i}-D_{i}\right) x_{i}+l_{i} y_{i i}\right] \\
\text { s.t. } & \sum_{i \in[n]} z_{i} \leq k . \\
& y_{i i} \leq x_{i}, \forall i \in[n], \\
& y_{i i} \leq z_{i}, \forall i \in[n], \\
& z_{i}, x_{i} \in\{0,1\}, y_{i i} \geq 0, \forall i \in[n] .
\end{aligned}
$$

We let $\widehat{\Xi}_{C}$ denote the continuous relaxation of the feasible region of Model 41], where we $\operatorname{relax} \boldsymbol{x}, \boldsymbol{z}$ to be continuous. Then, it is sufficient to show that $\widehat{\Xi}_{C}$ is an integral polytope.

First of all, let us write the constraints $41 \mathrm{~b}-41 \mathrm{e}$ in the matrix form as below:

$$
\begin{aligned}
& {\left[\begin{array}{ccc}
\boldsymbol{e}^{\top} & \mathbf{0} & \mathbf{0} \\
\mathbf{0} & \boldsymbol{I} & -\boldsymbol{I} \\
-\boldsymbol{I} & \boldsymbol{I} & \mathbf{0}
\end{array}\right]\left[\begin{array}{l}
\boldsymbol{z} \\
\boldsymbol{y} \\
\boldsymbol{x}
\end{array}\right] \leq\left[\begin{array}{l}
k \\
\mathbf{0} \\
\mathbf{0}
\end{array}\right],} \\
& \text { where } \boldsymbol{z}=\left[\begin{array}{c}
z_{1} \\
z_{2} \\
\vdots \\
z_{n}
\end{array}\right], \boldsymbol{y}=\left[\begin{array}{c}
y_{11} \\
y_{22} \\
\vdots \\
y_{n n}
\end{array}\right], \boldsymbol{x}=\left[\begin{array}{c}
x_{1} \\
x_{2} \\
\vdots \\
x_{n}
\end{array}\right] \text {. By Theorem } 19.3 \text { on Page } 269 \text { of Schrijver (1998), }
\end{aligned}
$$


to prove $\widehat{\Xi}_{C}$ is a integral polytope, it is sufficient to prove $\boldsymbol{A}=\left[\begin{array}{ccc}\boldsymbol{e}^{\top} & \mathbf{0} & \mathbf{0} \\ \mathbf{0} & \boldsymbol{I} & -\boldsymbol{I} \\ -\boldsymbol{I} & \boldsymbol{I} & \mathbf{0}\end{array}\right]$ is totally unimodular (TU). Indeed, if $\boldsymbol{A}$ is TU, and since $\left[\begin{array}{l}k \\ \mathbf{0} \\ \mathbf{0}\end{array}\right]$ is integral, thus, $\widehat{\Xi}_{C}$ is an integral polytope. Hence, the continuous relaxation of Model (41), which is a linear program, has an integral optimal solution. According to Theorem 19.3 on Page 269 of Schrijver (1998), to prove $\boldsymbol{A}$ is a totally unimodular matrix, it is sufficient to prove that for any $S \subseteq[3 n]$, there exist $S_{1}$ and $S_{2}$ such that $S_{1} \cap S_{2}=\emptyset, S_{1} \cup S_{2}=S, \sum_{i \in S_{1}} \boldsymbol{A}_{. i}-\sum_{i \in S_{2}} \boldsymbol{A}_{. i} \in\{-1,0,1\}^{2 n+1}$. We let $\widehat{S}_{1}=S \cap\{1,2, \cdots, n\}=\left\{i_{1}, \cdots, i_{\left|\bar{S}_{1}\right|}\right\}, T_{z}^{1}=\left\{i_{\tau}\right\}_{\tau \leq\left|\bar{S}_{1}\right|, \tau \text { is odd }}$ and $T_{z}^{2}=\left\{i_{\tau}\right\}_{\tau \leq\left|\bar{S}_{1}\right|, \tau \text { is even }}$. Also, we let $\widehat{S}_{2}=S \cap\{n+1, \cdots, 2 n\}, \widehat{S}_{3}=S \cap\{2 n+1, \cdots, 3 n\}, T_{y}^{1}=\left\{j \in \widehat{S}_{2}: j-n \in T_{z}^{1}\right\}$, $T_{y}^{2}=\widehat{S}_{2} \backslash T_{y}^{1}, T_{x}^{1}=\left\{j \in \widehat{S}_{3}: j-n \in T_{y}^{1}\right\}, T_{x}^{2}=\bar{S}_{3} \backslash T_{x}^{1}$. Clearly, we have $S_{1}=T_{z}^{1} \cup T_{y}^{1} \cup T_{x}^{1}$, $S_{2}=S \backslash S_{1}$. For such $S_{1}$ and $S_{2}$, we have $\sum_{i \in S_{1}} \boldsymbol{A}_{. i}-\sum_{i \in S_{2}} \boldsymbol{A}_{. i} \in\{-1,0,1\}^{2 n+1}$.

$k=n$. From the discussion in Section 4.3 , we already know at the optimality, we must have $z_{i}^{*}=1$ for all $i \in[n]$ when $k=n$. In (22a), the coefficient of $y_{j i}$ is $\sum_{j \in[n]} \underline{\alpha}_{j i}\left(\psi_{j}-u_{j}\right) \geq 0$, since $\psi_{j} \geq u_{j}$ for each $j, i \in[n]$ and $j \neq i$. Also, the coefficient of $y_{i i}$ is $l_{i}$, which is nonnegative, for each $i \in[n]$. Thus, at the optimality of the continuous relaxation of Model (22), we must have $y_{j i}=\min \left(x_{i}, z_{j}\right)=\min \left(x_{i}, 1\right)=x_{i}$ for all $i, j \in[n]$. Then, the continuous relaxation of Model (22) is equivalent to

$$
\begin{aligned}
& \max _{\boldsymbol{x} \in[0,1]^{n}} \sum_{i \in[n]} \bar{S}_{i}\left(\left(Q_{i}-D_{i}\right) x_{i}+l_{i} y_{i i}-\sum_{j \in[n]} \underline{\alpha}_{j i}\left(u_{j} y_{j i}+\psi_{j}\left(x_{i}-y_{j i}\right)\right)\right) \\
= & \max _{x \in[0,1]^{n}} \sum_{i \in[n]} \bar{S}_{i}\left(\left(Q_{i}-D_{i}+l_{i}\right)+\sum_{j \in[n]} \underline{\alpha}_{j i}\left(Q_{j}-D_{j}+l_{j}\right)_{+}\right) x_{i},
\end{aligned}
$$

which is a linear program over a unit box. Thus, there exists an optimal solution $\boldsymbol{x}^{*}$ of the above linear program, which corresponds to an extreme point of the box $[0,1]^{n}$, i.e., $x_{i}^{*} \in\{0,1\}$ for all $i \in[n]$. Thus, $y_{j i}^{*}=x_{i}^{*} \in\{0,1\}$ for all $i, j \in[n]$. Therefore, the continuous relaxation of Model 222 has an integral optimal solution $\left(\boldsymbol{x}^{*}, \boldsymbol{y}^{*}, \boldsymbol{z}^{*}\right)$. 


\section{Appendix B. MILP Reformulation of Model 20 for General $k^{\alpha}$}

Suppose $k^{\alpha}$ is general, i.e., Assumption 1 does not hold. Then the formulation 20 becomes

$$
\begin{aligned}
R(\boldsymbol{Q}, \boldsymbol{u}, \boldsymbol{\psi})=\max _{\boldsymbol{z}} & \sum_{i \in[n]} \bar{S}_{i}\left[Q_{i}-D_{i}+l_{i} z_{i}-\sum_{j \in[n]}\left(\alpha_{j i}-l_{j i}^{\alpha} z_{j i}^{\alpha}\right)\left(u_{j} z_{j}+\psi_{j}\left(1-z_{j}\right)\right)\right]_{+}, \\
\text {s.t. } & \sum_{i \in[n]} z_{i} \leq k, \\
& \sum_{j \in[n]} \sum_{i \in[n]} z_{j i}^{\alpha} \leq k^{\alpha}, \\
& z_{i} \in\{0,1\}, \forall i \in[n], \\
& z_{j i}^{\alpha} \in\{0,1\}, \forall j, i \in[n] .
\end{aligned}
$$

Similar to Proposition 3, We can reformulate (43) as an MILP.

Proposition 4. The inner maximization problem (43) is equivalent to

$$
\begin{aligned}
R(\boldsymbol{Q}, \boldsymbol{u}, \boldsymbol{\psi})=\max _{\boldsymbol{x}, \boldsymbol{y}, \boldsymbol{z}} & \sum_{i \in[n]} \bar{S}_{i}\left\{\left(Q_{i}-D_{i}\right) x_{i}+l_{i} y_{i i}-\sum_{j \in[n]} \alpha_{j i}\left[u_{j} y_{j i}+\psi_{j}\left(x_{i}-y_{j i}\right)\right]\right. \\
& \left.+\sum_{j \in[n]} l_{j i}^{\alpha}\left[u_{j} T_{j i}^{\alpha}+\psi_{j}\left(B_{j i}^{\alpha}-T_{j i}^{\alpha}\right)\right]\right\} \\
\text { s.t. } \quad & \sum_{i \in[n]} z_{i} \leq k . \\
& \sum_{j \in[n]} \sum_{i \in[n]} z_{j i}^{\alpha} \leq k^{\alpha}, \\
& y_{j i} \leq x_{i}, \quad y_{j i} \leq z_{j}, \forall i, j \in[n], \\
& T_{j i}^{\alpha} \leq z_{j i}^{\alpha}, T_{j i}^{\alpha} \leq y_{j i}, \forall i, j \in[n], \\
& B_{j i}^{\alpha} \leq z_{j i}^{\alpha}, B_{j i}^{\alpha} \leq x_{i}, B_{j i}^{\alpha} \geq z_{j i}^{\alpha}+x_{i}-1, \forall i, j \in[n] \\
& z_{j i}^{\alpha}, z_{i}, x_{i} \in\{0,1\}, y_{j i}, T_{j i}^{\alpha} \geq 0, \forall i, j \in[n] .
\end{aligned}
$$

Proof. The proof is similar to that of Proposition 3 , i.e., we eliminate the bilinear terms by introducing variables $y_{j i}=x_{i} z_{j}, T_{j i}^{\alpha}=z_{j i}^{\alpha} y_{j i}, B_{j i}^{\alpha}=z_{j i}^{\alpha} x_{i}$ for each $i, j \in[n]$, and then applying McCormick inequalities (McCormick, 1976).

Let us define $J\left(\boldsymbol{Q}, \boldsymbol{u}, \boldsymbol{\psi}, \boldsymbol{x}, \boldsymbol{y}, \boldsymbol{z}, \boldsymbol{T}^{\boldsymbol{\alpha}}, \boldsymbol{B}^{\boldsymbol{\alpha}}, \boldsymbol{z}^{\boldsymbol{\alpha}}\right)$ to be the objective function of (44), i.e.,

$$
J\left(\boldsymbol{Q}, \boldsymbol{u}, \boldsymbol{\psi}, \boldsymbol{x}, \boldsymbol{y}, \boldsymbol{z}, \boldsymbol{T}^{\boldsymbol{\alpha}}, \boldsymbol{B}^{\boldsymbol{\alpha}}, \boldsymbol{z}^{\boldsymbol{\alpha}}\right)=\sum_{i \in[n]} \bar{S}_{i}\left\{\left(Q_{i}-D_{i}\right) x_{i}+l_{i} y_{i i}-\sum_{j \in[n]} \alpha_{j i}\left[u_{j} y_{j i}+\psi_{j}\left(x_{i}-y_{j i}\right)\right]\right.
$$




$$
\left.+\sum_{j \in[n]} l_{j i}^{\alpha}\left[u_{j} T_{j i}^{\alpha}+\psi_{j}\left(B_{j i}^{\alpha}-T_{j i}^{\alpha}\right)\right]\right\} .
$$

And $\Lambda=\left\{\left(\boldsymbol{x}, \boldsymbol{y}, \boldsymbol{z}, \boldsymbol{T}^{\boldsymbol{\alpha}}, \boldsymbol{B}^{\boldsymbol{\alpha}}, \boldsymbol{z}^{\boldsymbol{\alpha}}\right)\right.$; 44a $\left.\left.-44 \mathrm{~h}\right)\right\}$. According to Section 5.2 , we have the following equivalent MILP formulation of R-MNMS 44:

$$
\begin{aligned}
v^{*}=\max _{\boldsymbol{Q}, \boldsymbol{u}, \boldsymbol{\psi}, \boldsymbol{\chi}, \boldsymbol{w}, \vartheta} & \sum_{i \in[n]} \bar{P}_{i} Q_{i}-\vartheta, \\
\text { s.t. } & \vartheta \geq J\left(\boldsymbol{Q}, \boldsymbol{u}, \boldsymbol{\psi}, \boldsymbol{x}, \boldsymbol{y}, \boldsymbol{z}, \boldsymbol{T}^{\boldsymbol{\alpha}}, \boldsymbol{B}^{\boldsymbol{\alpha}}, \boldsymbol{z}^{\boldsymbol{\alpha}}\right), \forall\left(\boldsymbol{x}, \boldsymbol{y}, \boldsymbol{z}, \boldsymbol{T}^{\boldsymbol{\alpha}}, \boldsymbol{B}^{\boldsymbol{\alpha}}, \boldsymbol{z}^{\boldsymbol{\alpha}}\right) \in \Lambda \\
& w_{i}^{(e)}, u_{i}, \psi_{i} \geq 0, \chi_{i}^{(e)} \in\{0,1\}, \forall i \in[n], e \in[3] . \\
& \text { 23a }-23 \mathrm{a} .
\end{aligned}
$$

Appendix C. Computational Results of Branch and Cut Algorithm and Conservative Approximation Method with $n=10$ 


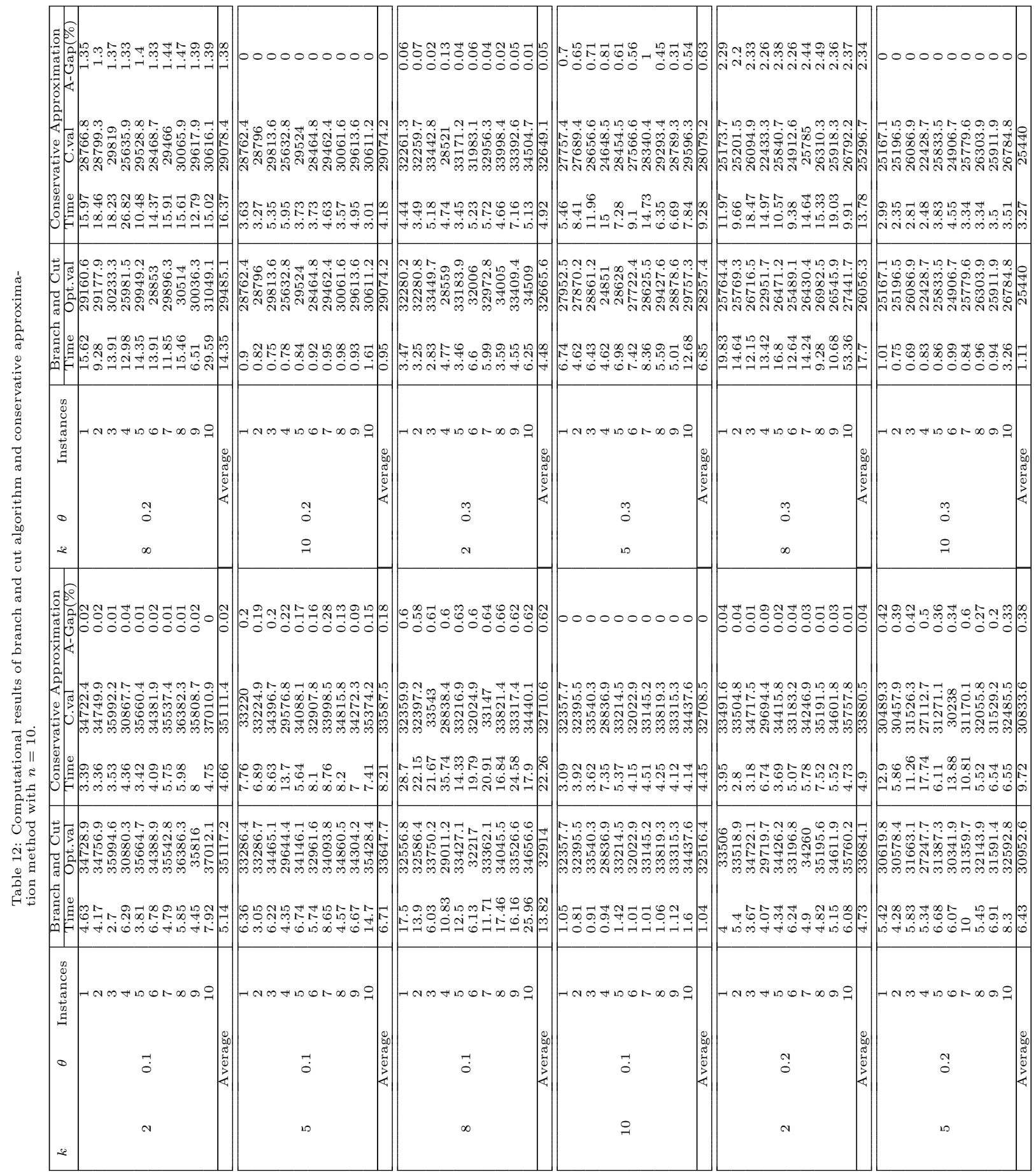




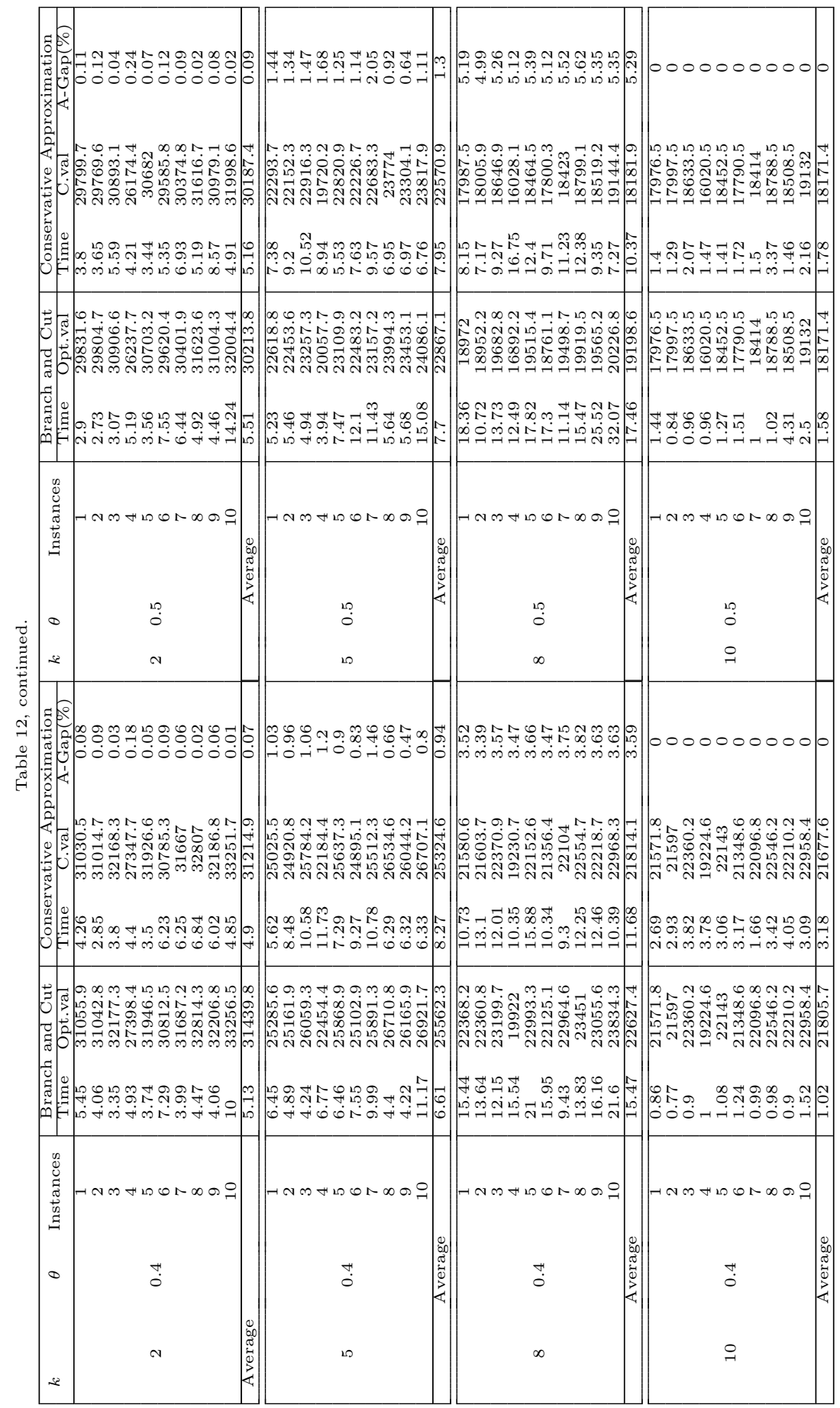




\section{Appendix D. Illustration of Finding the Best Pair of Budgets of Uncertainty}

(i.e., $k^{*}, k^{\alpha *}$ )

In this Appendix, we illustrate how to find the best pair of budgets of uncertainty (i.e., $k^{*}, k^{\alpha *}$ ) and also test robustness of Model (45). Suppose that there are 10 products. The values of $\boldsymbol{p}, \boldsymbol{c}$, and $s$ are the same as those in Section 6.1. We also assumed that there are 200 historical data of demand and substitution rates and we split them into two groups, $\Upsilon_{1}, \Upsilon_{2}$, with equal size. The historical demand were generated by sampling from independent uniformly random variables between 20 and 80 . The historical substitution rates were generated by sampling from independent uniformly random variables between 0.05 and 0.1 . We choose the candidate set $\mathcal{K}$ of budget of uncertainty $k$ and $k^{\alpha}$ as $\mathcal{K}=\{0,1, \cdots, 10\} \times\{0,10, \cdots, 90\}$. According to Section 2.2 with percentile $q=10$, $w_{1}=\frac{1}{n}=\frac{1}{10}$, and $w_{2}=\frac{1}{n^{2}-n}=\frac{1}{90}$, we found the optimal budget of uncertainty $k^{*}=9$ and $k^{\alpha *}=0$, which is the smallest $w_{1} k+w_{2} k^{\alpha}=0.9$ such that $v^{*}\left(k, k^{\alpha}\right) \leq \widehat{\Pi}^{10 \%}\left(k, k^{\alpha}\right)$ as shown in Table 13 . 
Table 13: The 10th percentile of profits for Model (2) by plugging in the optimal order quantities of robust model (45) for different $k$ and $k^{\alpha}$. The best pair of budgets of uncertainty (i.e., $k^{*}, k^{\alpha *}$ ) is highlighted in bold.

\begin{tabular}{|c|c|c|c|c|c|c|c|c|c|c|c|}
\hline$k$ & $k^{\alpha}$ & $v^{*}\left(k, k^{\alpha}\right)$ & $\widehat{\Pi}^{10 \%}\left(k, k^{\alpha}\right)$ & $k$ & $k^{\alpha}$ & $v^{*}\left(k, k^{\alpha}\right)$ & $\widehat{\Pi}^{10 \%}\left(k, k^{\alpha}\right)$ & $k$ & $k^{\alpha}$ & $v^{*}\left(k, k^{\alpha}\right)$ & $\widehat{\Pi}^{10 \%}\left(k, k^{\alpha}\right)$ \\
\hline 0 & 0 & 24648.0 & 6075.4 & 3 & 70 & 17841.1 & 6245.2 & 7 & 40 & 9792.1 & 11009.3 \\
\hline 0 & 10 & 24648.0 & 6075.4 & 3 & 80 & 17840.5 & 6249.8 & 7 & 50 & 9363.0 & 6705.8 \\
\hline 0 & 20 & 24648.0 & 6075.4 & 3 & 90 & 17841.0 & 6250.7 & 7 & 60 & 9351.1 & 6712.8 \\
\hline 0 & 30 & 24648.0 & 6075.4 & 4 & 0 & 15836.1 & 6354.0 & 7 & 70 & 9339.8 & 6713.5 \\
\hline 0 & 40 & 24648.0 & 6075.4 & 4 & 10 & 15765.7 & 6366.1 & 7 & 80 & 9333.2 & 6730.5 \\
\hline 0 & 50 & 24648.0 & 6075.4 & 4 & 20 & 15708.3 & 6353.9 & 7 & 90 & 9332.4 & 6734.8 \\
\hline 0 & 60 & 24648.0 & 6075.4 & 4 & 30 & 15687.0 & 6310.7 & 8 & 0 & 11017.2 & 10938.2 \\
\hline 0 & 70 & 24648.0 & 6075.4 & 4 & 40 & 15682.5 & 6331.6 & 8 & 10 & 10450.6 & 10933.6 \\
\hline 0 & 80 & 24648.0 & 6075.4 & 4 & 50 & 15681.1 & 6314.6 & 8 & 20 & 9893.4 & 10923.7 \\
\hline 0 & 90 & 24648.0 & 6075.4 & 4 & 60 & 15679.0 & 6318.5 & 8 & 30 & 9345.4 & 10909.2 \\
\hline 1 & 0 & 22350.7 & 6102.6 & 4 & 70 & 15676.7 & 6322.5 & 8 & 40 & 8810.5 & 10873.5 \\
\hline 1 & 10 & 22340.4 & 6088.8 & 4 & 80 & 15674.5 & 6340.7 & 8 & 50 & 8284.9 & 10920.2 \\
\hline 1 & 20 & 22340.3 & 6088.8 & 4 & 90 & 15674.1 & 6339.1 & 8 & 60 & 7765.2 & 10947.1 \\
\hline 1 & 30 & 22340.2 & 6088.9 & 5 & 0 & 13763.6 & 6449.4 & 8 & 70 & 7479.0 & 7479.0 \\
\hline 1 & 40 & 22340.0 & 6089.0 & 5 & 10 & 13679.6 & 6455.3 & 8 & 80 & 7479.0 & 7479.0 \\
\hline 1 & 50 & 22339.9 & 6089.0 & 5 & 20 & 13612.4 & 6461.6 & 8 & 90 & 7479.0 & 7479.0 \\
\hline 1 & 60 & 22339.3 & 6089.4 & 5 & 30 & 13567.9 & 6497.8 & 9 & 0 & 10066.3 & 10066.3 \\
\hline 1 & 70 & 22339.7 & 6089.1 & 5 & 40 & 13553.3 & 6435.2 & 9 & 10 & 9460.2 & 10060.5 \\
\hline 1 & 80 & 22339.7 & 6089.1 & 5 & 50 & 13546.6 & 6442.1 & 9 & 20 & 8864.2 & 10048.2 \\
\hline 1 & 90 & 22339.7 & 6089.1 & 5 & 60 & 13543.8 & 6437.7 & 9 & 30 & 8277.9 & 10030.0 \\
\hline 2 & 0 & 20119.1 & 6203.6 & 5 & 70 & 13541.0 & 6433.5 & 9 & 40 & 7705.0 & 9990.0 \\
\hline 2 & 10 & 20076.7 & 6141.4 & 5 & 80 & 13538.3 & 6428.9 & 9 & 50 & 7138.4 & 9966.7 \\
\hline 2 & 20 & 20069.8 & 6119.3 & 5 & 90 & 13537.9 & 6456.2 & 9 & 60 & 6634.7 & 6634.7 \\
\hline 2 & 30 & 20070.5 & 6118.8 & 6 & 0 & 12589.8 & 10730.7 & 9 & 70 & 6634.7 & 6634.7 \\
\hline 2 & 40 & 20070.1 & 6119.4 & 6 & 10 & 12089.1 & 10723.4 & 9 & 80 & 6634.7 & 6634.7 \\
\hline 2 & 50 & 20069.4 & 6123.4 & 6 & 20 & 11605.0 & 9930.4 & 9 & 90 & 6634.7 & 6634.7 \\
\hline 2 & 60 & 20069.8 & 6118.6 & 6 & 30 & 11493.7 & 6579.1 & 10 & 0 & 8972.8 & 8972.8 \\
\hline 2 & 70 & 20067.8 & 6125.6 & 6 & 40 & 11460.1 & 6582.3 & 10 & 10 & 8329.4 & 8965.3 \\
\hline 2 & 80 & 20067.9 & 6125.8 & 6 & 50 & 11445.4 & 6587.3 & 10 & 20 & 7710.8 & 8936.0 \\
\hline 2 & 90 & 20068.2 & 6122.1 & 6 & 60 & 11437.4 & 6594.0 & 10 & 30 & 7103.4 & 8919.8 \\
\hline 3 & 0 & 17959.3 & 6266.8 & 6 & 70 & 11433.2 & 6594.8 & 10 & 40 & 6503.4 & 8889.2 \\
\hline 3 & 10 & 17895.1 & 6259.5 & 6 & 80 & 11430.0 & 6594.0 & 10 & 50 & 5910.2 & 8868.3 \\
\hline 3 & 20 & 17856.2 & 6255.4 & 6 & 90 & 11429.3 & 6612.1 & 10 & 60 & 5790.4 & 5790.4 \\
\hline 3 & 30 & 17847.3 & 6257.0 & 7 & 0 & 11851.7 & 11015.7 & 10 & 70 & 5790.4 & 5790.4 \\
\hline 3 & 40 & 17844.2 & 6270.3 & 7 & 10 & 11319.8 & 11018.2 & 10 & 80 & 5790.4 & 5790.4 \\
\hline 3 & 50 & 17844.4 & 6271.2 & 7 & 20 & 10796.7 & 11022.8 & 10 & 90 & 5790.4 & 5790.4 \\
\hline 3 & 60 & 17841.8 & 6265.4 & 7 & 30 & 10287.6 & 11019.3 & & & & \\
\hline
\end{tabular}




\section{Appendix E. Proof of the Pearson Correlation Coefficient}

Given i.i.d. uniform random variables $\left\{\tilde{U}_{i}\right\}_{i \in\{0\} \cup[n]}$ distributed between -1 and 1 , suppose that the random demand of $i$ th product is $\tilde{D}_{i}=50+30\left(\widehat{\rho} \tilde{U}_{0}+(1-\widehat{\rho}) \tilde{U}_{i}\right)$ for each $i \in[n]$. The Pearson correlation coefficient between any two distinct products $i, j \in[n]$ such that $i \neq j$ is equal to $\widehat{\rho}^{2} /\left(\widehat{\rho}^{2}+(1-\widehat{\rho})^{2}\right)$ and it is increasing with the increase of $\widehat{\rho}$ when $\widehat{\rho} \in[0,1]$.

Indeed, we let $\mathbb{E}(\cdot)$ and $\mathbb{V}(\cdot)$ denote the expectation and variance operators, respectively. Since $\left\{\tilde{U}_{i}\right\}_{i \in\{0\} \cup[n]}$ are i.i.d. and uniformly distributed in [-1, 1], we have

$$
\mathbb{E}\left(\tilde{U}_{i}\right)=0, \mathbb{V}\left(\tilde{U}_{i}\right)=1 / 3 .
$$

For each product $i \in[n]$, the expectation of $\tilde{D}_{i}$ is equal to

$$
\begin{aligned}
\mathbb{E}\left(\tilde{D}_{i}\right) & =\mathbb{E}\left(50+30\left[\hat{\rho} \tilde{U}_{0}+(1-\widehat{\rho}) \tilde{U}_{i}\right)\right] \\
& =50+30\left[\widehat{\rho} \mathbb{E}\left(\tilde{U}_{0}\right)+(1-\widehat{\rho}) \mathbb{E}\left(\tilde{U}_{i}\right)\right] \\
& =50,
\end{aligned}
$$

while its variance is

$$
\begin{aligned}
\mathbb{V}\left(\tilde{D}_{i}\right) & =\mathbb{V}\left(50+30\left[\hat{\rho} \tilde{U}_{0}+(1-\widehat{\rho}) \tilde{U}_{i}\right)\right] \\
& =30^{2}\left[\widehat{\rho}^{2} \mathbb{V}\left(\tilde{U}_{0}\right)+(1-\widehat{\rho})^{2} \mathbb{V}\left(\tilde{U}_{i}\right)\right] \\
& =300\left(\widehat{\rho}^{2}+(1-\widehat{\rho})^{2}\right) .
\end{aligned}
$$

For any pair $i, j \in[10]$ with $i \neq j$, we have $\tilde{D}_{i}=50+30\left(\widehat{\rho} \tilde{U}_{0}+(1-\widehat{\rho}) \tilde{U}_{i}\right), \tilde{D}_{j}=50+30\left(\widehat{\rho} \tilde{U}_{0}+\right.$ $\left.(1-\widehat{\rho}) \tilde{U}_{j}\right)$. Then the covariance between $\tilde{D}_{i}$ and $\tilde{D}_{j}$ is

$$
\begin{aligned}
\operatorname{Cov}\left(\tilde{D}_{i}, \tilde{D}_{j}\right) & =\operatorname{Cov}\left(50+30\left(\widehat{\rho} \tilde{U}_{0}+(1-\widehat{\rho}) \tilde{U}_{i}\right), 50+30\left(\widehat{\rho} \tilde{U}_{0}+(1-\widehat{\rho}) \tilde{U}_{j}\right)\right) \\
& =900\left[\widehat{\rho}^{2} \mathbb{V}\left(\tilde{U}_{0}\right)+\rho(1-\widehat{\rho}) \operatorname{Cov}\left(\tilde{U}_{0}, \tilde{U}_{j}\right)+\rho(1-\widehat{\rho}) \operatorname{Cov}\left(\tilde{U}_{0}, \tilde{U}_{i}\right)+(1-\widehat{\rho})^{2} \operatorname{Cov}\left(\tilde{U}_{i}, \tilde{U}_{j}\right)\right] \\
& =300 \widehat{\rho}^{2}
\end{aligned}
$$

The last equality holds since $\tilde{U}_{0}, \tilde{U}_{i}, \tilde{U}_{j}$ are independent with zero means. Thus, the Pearson correlation coefficient between two distinct products $i$ and $j$ is equal to

$$
\frac{\operatorname{Cov}\left(\tilde{D}_{i}, \tilde{D}_{j}\right)}{\sqrt{\mathbb{V}\left(\tilde{D}_{i}\right)} \cdot \sqrt{\mathbb{V}\left(\tilde{D}_{j}\right)}}=\frac{300 \widehat{\rho}^{2}}{300\left(\widehat{\rho}^{2}+(1-\widehat{\rho})^{2}\right)}=\frac{\widehat{\rho}^{2}}{\widehat{\rho}^{2}+(1-\widehat{\rho})^{2}}=\frac{1}{1+\left(\frac{1}{\hat{\rho}}-1\right)^{2}}
$$

which is increasing with the increase of $\widehat{\rho}$ when $\widehat{\rho} \in[0,1]$. 